\title{
Hecke von Neumann Algebra of Ergodic Discrete Measured Equivalence Relations
}

\author{
by
}

\author{
Hisashi AOI and Takehiko YAmANOUCHI
}

\begin{abstract}
We generalize the notion of a Hecke pair of groups to the case of an inclusion of ergodic discrete measured equivalence relations. A key ingredient in defining this new concept is a commensurability subrelation introduced and discussed in 3 . As in the group case, with each such Hecke pair, we associate a von Neumann algebra which we call the Hecke von Neumann algebra of the pair. It is shown that the Hecke von Neumann algebra thus defined is realized as one of the relative commutants of the tower of the corresponding inclusion of factors.
\end{abstract}

2010 Mathematics Subject Classification: 46L10, 37A20, 33D80.

Keywords: measured equivalence relation, Hecke pair, von Neumann algebra.

\section{§1. Introduction}

This paper is concerned with a certain type of Borel subrelations of general ergodic discrete measured equivalence relations. In [3], we introduced and investigated a notion of the commensurability groupoid $\mathcal{C G}(B)$ for an inclusion of (separable) factors $B \subseteq A$ which have a common Cartan subalgebra $D$. Our motivation to study this new object stems from the work [13] of Izumi-Longo-Popa, who introduced an extremely important concept of discreteness of an inclusion of factors $N \subseteq M$, in order to analyze such a situation as would arise when $N$ is realized as the fixed-point algebra of a minimal action of a compact group (Kac algebra) on $M$. Given an inclusion $B \subseteq A$ as stated above, we defined $\mathcal{C G}(B)$ to be a set of partial isometries in $A$ whose initial and final projections both belong to $B$

Communicated by H. Okamoto. Received November 2, 2009. Revised January 26, 2010.

H. Aoi: Department of Mathematical Sciences, Ritsumeikan University, 1-1-1 Nojihigashi, Kusatsu, Shiga, 525-8577, Japan;

e-mail: aoi@fc.ritsumei.ac.jp

T. Yamanouchi: Department of Mathematics, Tokyo Gakugei University, Koganei,

Tokyo 184-8501, Japan;

e-mail: tyamanou@u-gakugei.ac.jp 
satisfying a certain condition (see Subsection 2.3 for its precise definition). We proved in 3, among other results, that the inclusion $B \subseteq \mathcal{C G}(B)^{\prime \prime}$ is always discrete in the sense of Izumi-Longo-Popa, and that $\mathcal{C G}(B)^{\prime \prime}$ is the largest among the intermediate subfactors $C$ of $B \subseteq A$ such that $B \subseteq C$ is discrete.

Furthermore, according to [7, there exist an ergodic discrete measured equivalence relation $\mathcal{R}$ on a standard probability space $(X, \mu)$ and a 2-cocycle $\omega$ on $\mathcal{R}$ such that the inclusion $D \subseteq A$ is $*$-isomorphic to $W^{*}(X) \subseteq W^{*}(\mathcal{R}, \omega)$, where $W^{*}(\mathcal{R}, \omega)$ is a sort of matrix algebra over $\mathcal{R}$ twisted by $\omega$, and $W^{*}(X)$ is the subalgebra of diagonal matrices. Moreover, thanks to [1, there is an ergodic Borel subrelation $\mathcal{S}$ of $\mathcal{R}$ such that $B=W^{*}(\mathcal{S}, \omega)$. Therefore, discreteness of $B \subseteq A$ in our setting can be considered to be a condition for the inclusion of equivalence relations $\mathcal{S} \subseteq \mathcal{R}$. By [1] again, the subfactor $\mathcal{C G}(B)^{\prime \prime}$ has the form $\mathcal{C G}(B)^{\prime \prime}=W^{*}\left(\operatorname{Comm}_{\mathcal{R}}(\mathcal{S}), \omega\right)$ for a (unique) ergodic intermediate Borel subrelation $\operatorname{Comm}_{\mathcal{R}}(\mathcal{S})$ of $\mathcal{S} \subseteq \mathcal{R}$. The theorem in [3] cited above then tells us that $B \subseteq A$ is discrete if and only if $\operatorname{Comm}_{\mathcal{R}}(\mathcal{S})$ equals $\mathcal{R}$ up to a null set. We call $\operatorname{Comm}_{\mathcal{R}}(\mathcal{S})$ the commensurability subrelation of $\mathcal{S}$ in $\mathcal{R}$. This terminology is borrowed from group theory, as the next example shows. Suppose that the equivalence relations $\mathcal{S} \subseteq \mathcal{R}$ in question are derived from outer actions of countable discrete groups $H \subseteq G$ on an ergodic equivalence relation $\mathcal{P}$, i.e., $\mathcal{S}=H \ltimes \mathcal{P}$ and $\mathcal{R}=G \ltimes \mathcal{P}$ (see Section 11 below for details). It is (implicitly) stated in [13] that $\operatorname{Comm}_{\mathcal{R}}(\mathcal{S})$ in this case is equal to $\operatorname{Comm}_{G}(H) \ltimes \mathcal{P}$, where $\operatorname{Comm}_{G}(H):=\left\{g \in G:\left[H: H \cap g H g^{-1}\right]<\infty,\left[H: H \cap g^{-1} H g\right]<\infty\right\}$. The subgroup $\operatorname{Comm}_{G}(H)$ is often called the commensurability subgroup of $H$ in $G$. So discreteness of $B \subseteq A$ in this example is equivalent to $\operatorname{Comm}_{G}(H)$ being equal to $G$, in which case $H$ is said to be almost normal in $G$, or $(G, H)$ is called a Hecke pair. Suggested by this example, we generally say that an inclusion of ergodic discrete measure equivalence relations $\mathcal{S} \subseteq \mathcal{R}$ is a Hecke pair if $\operatorname{Comm}_{\mathcal{R}}(\mathcal{S})=\mathcal{R}$. It is well-known that the notion of a Hecke pair with the Hecke algebra of groups is extremely important in the theory of modular forms (see [17] for example). This notion has also attracted a lot of attention of operator algebraists since the pioneering work [4] of Bost and Connes on Hecke $C^{*}$-algebras. Hence we strongly believe that Hecke pairs of equivalence relations equally deserve a serious investigation. This is the topic we treat in the present article.

The organization of this paper is as follows.

Section 2 is devoted to preparations. We briefly recall the definitions of von Neumann algebras associated with discrete equivalence relations on standard measure spaces, the basic extension of an inclusion of factors and commensurability subrelations.

In Section 3, starting from an inclusion of ergodic equivalence relations $\mathcal{S} \subseteq \mathcal{R}$ on a measure space $X$, we identify the basic extension $A_{1}$ of the inclu- 
sion $B:=W^{*}(\mathcal{S}, \omega) \subseteq A:=W^{*}(\mathcal{R}, \omega)$ with the von Neumann algebra of the skew-product equivalence relation $I_{\sigma} \times \mathcal{R}$ derived from an index cocycle of $\mathcal{S} \subseteq \mathcal{R}$. Then we realize, through this identification, the relative commutant $A_{1} \cap B^{\prime}$ as the functions on $I \times X$ with a certain property (Theorem 3.2p. From Section 3 up to Section 8 , we devote ourselves to studying in detail the relative commutants $A_{1} \cap B$ and $A_{2} \cap A^{\prime}$ in the tower of factors $B \subseteq A \subseteq A_{1} \subseteq A_{2}$. The reason for this investigation is based upon a general philosophy that these relative commutants are in some sense "dual objects" to each other. In fact it will be shown in Section 8 as a consequence of analysis made in the preceding sections that if $(\mathcal{R}, \mathcal{S})$ is a Hecke pair in our sense, then one can construct a unitary, which we call the Fourier transform, between the Hilbert spaces on which $A_{1} \cap B$ and $A_{2} \cap A^{\prime}$ act standardly (Theorems 8.7 and 8.8. This result is a generalization of the Fourier transform on discrete groups.

In Section 9, we construct, from a given Hecke pair $(\mathcal{R}, \mathcal{S})$, a von Neumann algebra $\mathcal{H}^{*}(\mathcal{R}, \mathcal{S})$, which we term the Hecke von Neumann algebra associated with $(\mathcal{R}, \mathcal{S})$. The construction is a natural generalization of that of a Hecke $C^{*}$ algebra.

In Section 10, we show that if $(\mathcal{R}, \mathcal{S})$ is a Hecke pair, then the relative commutant $A_{2} \cap A^{\prime}$ considered above is $*$-isomorphic to the Hecke von Neumann algebra $\mathcal{H}^{*}(\mathcal{R}, \mathcal{S})$. This would mean that our definitions of a Hecke pair and a Hecke von Neumann algebra are right ones (Theorem 10.2 .

In Section 11, we discuss a relation between Hecke pairs of groups and those of equivalence relations. We show that every von Neumann algebra of a group Hecke pair can be obtained as $\mathcal{H}^{*}(\mathcal{R}, \mathcal{S})$ for a suitable pair $(\mathcal{R}, \mathcal{S})$.

Section 12 exhibits a few examples of Hecke pairs of equivalence relations.

\section{§2. Preliminaries}

In this section, we introduce symbols that will be repeatedly used in the whole of this paper. We also collect basic facts about discrete measured equivalence relations and Jones' basic extension of an inclusion of factors, which are necessary for our later discussion. The readers are referred to [3], 6], [7], [8], [13] regarding these topics.

We assume that all von Neumann algebras in this paper have separable preduals. For a (separable) Hilbert space $H$, we let $B(H)$ denote the algebra of all bounded operators on $H$.

For a faithful normal semifinite weight $\phi$ on a von Neumann algebra $M$, we set

$$
\mathfrak{n}_{\phi}:=\left\{x \in M: \phi\left(x^{*} x\right)<\infty\right\}, \quad \mathfrak{m}_{\phi}:=\mathfrak{n}_{\phi}^{*} \mathfrak{n}_{\phi}, \quad \mathfrak{m}_{\phi}^{+}:=\mathfrak{m}_{\phi} \cap M_{+} .
$$


More generally, for an operator valued weight $T$ ([9], [10], 18]) from a von Neumann algebra $M$ to a von Neumann subalgebra $N$, we set

$$
\mathfrak{n}_{T}:=\left\{x \in M: T\left(x^{*} x\right) \in N_{+}\right\}, \quad \mathfrak{m}_{T}:=\mathfrak{n}_{T}^{*} \mathfrak{n}_{T}, \quad \mathfrak{m}_{T}^{+}:=\mathfrak{m}_{T} \cap M_{+} .
$$

The Hilbert space obtained from $\phi$ by the GNS-construction will be denoted by $H_{\phi}$, and we let $\Lambda_{\phi}: \mathfrak{n}_{\phi} \rightarrow H_{\phi}$ stand for the natural injection.

\section{§2.1. Discrete measured equivalence relations}

Throughout this paper, we fix a discrete measured equivalence relation $\mathcal{R}$ on a standard probability space $(X, \mathfrak{B}, \mu)$ in which $\mu$ is quasi-invariant for $\mathcal{R}$. We denote by $\nu$ the $(\sigma$-finite) measure on $\mathcal{R}$ given by

$$
\nu(E):=\int_{X}\left|r^{-1}(\{x\}) \cap E\right| d \mu(x) \quad(E \text { a Borel subset of } \mathcal{R}),
$$

where $r: \mathcal{R} \rightarrow X$ is the projection onto the first coordinate, and $|S|$ in general stands for the cardinality of a (countable) set $S$. The Radon-Nikodym derivative associated with this measured equivalence relation will be denoted by $\delta$.

We also fix a (normalized) Borel 2-cocycle $\omega$ from $\mathcal{R}$ into the one-dimensional torus $\mathbb{T}$ in what follows. We then write $W^{*}(\mathcal{R}, \omega)$ for the von Neumann algebra on the Hilbert space $L^{2}(\mathcal{R}, \nu)$ obtained by the Feldman-Moore construction from $\mathcal{R}$ and $\omega$. Briefly, the construction is as follows. We first define the subspace $\mathfrak{A}_{I}$ of $L^{2}(\mathcal{R}, \nu)$ by

$$
\mathfrak{A}_{I}:=\left\{\xi \in L^{2}(\mathcal{R}, \nu): \xi \text { is } \delta \text {-bounded and }\|\xi\|_{I}<\infty\right\} .
$$

See [11 and 23] for the definition and properties of $\mathfrak{A}_{I}$ and for the terminology used above. We then introduce a product and an involution on $\mathfrak{A}_{I}$ as follows:

$$
(f * g)(x, z):=\sum_{y \sim x} f(x, y) g(y, z) \omega(x, y, z), \quad f^{\sharp}(x, z):=\delta(x, z)^{-1} \overline{f(z, x)},
$$

where the sum is over all $y$ equivalent to $x$. By the same argument as in [1] and [23, one can show that $\mathfrak{A}_{I}$ is a left Hilbert algebra (in fact, a Tomita algebra) in $L^{2}(\mathcal{R}, \nu)$. The left von Neumann algebra of $\mathfrak{A}_{I}$ is denoted by $W^{*}(\mathcal{R}, \omega)$. The modular operator $\Delta$ the modular conjugation $J$ are given by

$$
\Delta \xi:=\delta \xi, \quad\{J \xi\}(x, y)=\delta(x, y)^{-1 / 2} \overline{\xi(y, x)} \quad\left(\xi \in \mathfrak{A}_{I}\right) .
$$

The left multiplication by $f \in \mathfrak{A}_{I}$ will be denoted by $L^{\omega}(f): L^{\omega}(f) \xi:=f * \xi$. Remark that every element $a \in W^{*}(\mathcal{R}, \omega)$ can in fact be written as $a=L^{\omega}(f)$ for some $f \in L^{2}(\mathcal{R}, \nu)$. Moreover, for each $a_{i}:=L^{\omega}\left(f_{i}\right) \in W^{*}(\mathcal{R}, \omega)(i=1,2)$, we have $a_{i}^{*}=L^{\omega}\left(f_{i}^{\sharp}\right)$ and $a_{1} a_{2}=L^{\omega}\left(f_{1} * f_{2}\right)$. The abelian von Neumann algebra $L^{\infty}(X, \mu)$ 
is embedded into $W^{*}(\mathcal{R}, \omega)$ through the representation $f \in L^{\infty}(X, \mu) \mapsto f \circ r$. We will always identify $L^{\infty}(X, \mu)$ with its image $D$ under this representation. This algebra $D$ is called a Cartan subalgebra of $W^{*}(\mathcal{R}, \omega)$.

If $\mathcal{D}$ designates the diagonal subset $\{(x, x): x \in X\}$ of $\mathcal{R}$, then $\chi_{\mathcal{D}} \in L^{2}(\mathcal{R}, \nu)$ is a cyclic and separating unit vector for $W^{*}(\mathcal{R}, \omega)$. Here $\chi_{E}$ in general stands for the characteristic function of a set $E$. We will often simply write $\xi_{0}$ for this vector. Denote by $\theta$ the faithful vector state on $W^{*}(\mathcal{R}, \omega)$ determined by $\xi_{0}$; we will always identify $H_{\theta}$ with $L^{2}(\mathcal{R}, \nu)$.

We define $[\mathcal{R}]_{*}$ to be the set of all bimeasurable nonsingular transformations $\rho$ from a Borel subset $\operatorname{Dom}(\rho)$ of $X$ onto a Borel subset $\operatorname{Im}(\rho)$ of $X$ satisfying $(x, \rho(x)) \in \mathcal{R}$ for $\mu$-a.e. $x \in \operatorname{Dom}(\rho)$. For any $\rho \in[\mathcal{R}]_{*}$, set $\Gamma(\rho):=\{(x, \rho(x))$ : $x \in \operatorname{Dom}(\rho)\}$. Then, for each measurable function $g$ on $X$ of absolute value one, $L^{\omega}\left(\delta^{-1 / 2}(g \circ r) \chi_{\Gamma\left(\rho^{-1}\right)}\right)$ is a partial isometry in $W^{*}(\mathcal{R}, \omega)$ whose initial and final projections are respectively $\chi_{\operatorname{Dom}(\rho)}$ and $\chi_{\operatorname{Im}(\rho)}$. We denote such partial isometries in $W^{*}(\mathcal{R}, \omega)$ by $\mathcal{G N}(D)$.

For a Borel 1-cocycle $c$ from $\mathcal{R}$ into a (second countable) locally compact group $K$, the essential range of $c$ is the smallest closed subset $\sigma(c)$ of $K$ such that $c^{-1}(\sigma(c))$ has complement of measure zero. The asymptotic range $r^{*}(c)$ of $c$ is by definition $\bigcap\left\{\sigma\left(c_{B}\right): B(\subseteq X)\right.$ Borel and $\left.\mu(B)>0\right\}$, where $c_{B}$ stands for the restriction of $c$ to the reduction $\mathcal{R}_{B}:=\{(x, y) \in \mathcal{R}: x, y \in B\}$.

Assume now that $\mathcal{R}$ is ergodic. Let $\mathcal{S}$ be a Borel subrelation of $\mathcal{R}$. By 8 , we may choose a countable family $\left\{\psi_{i}\right\}_{i \in I}$ of Borel maps from $X$ into itself such that (i) $\left(x, \psi_{i}(x)\right) \in \mathcal{R}$ for all $i \in I$ and $\mu$-a.e. $x \in X$; (ii) for $\mu$-a.e. $x \in X,\left\{\mathcal{S}\left(\psi_{i}(x)\right)\right\}_{i \in I}$ is a partition of $\mathcal{R}(x)$, where $\mathcal{R}(x):=\{y \in X:(x, y) \in \mathcal{R}\}$. The family $\left\{\psi_{i}\right\}_{i \in I}$ is called choice functions for $\mathcal{S} \subseteq \mathcal{R}$. Once the choice functions $\left\{\psi_{i}\right\}_{i \in I}$ are fixed, we can define the index cocycle $\sigma: \mathcal{R} \rightarrow \Sigma(I)$ of the pair $\mathcal{S} \subseteq \mathcal{R}$, where $\Sigma(I)$ denotes the full permutation group on $I$, by the following rule:

$$
\sigma(x, y)(i)=j \Leftrightarrow\left(\psi_{i}(y), \psi_{j}(x)\right) \in \mathcal{S} .
$$

\section{§2.2. Basic extension}

Let $B \subseteq A$ be an inclusion of factors with a faithful normal conditional expectation $E_{B}$. (In the situation considered in the following sections, such an expectation always exists and is unique.) Fix a faithful normal state $\phi_{0}$ on $B$ and set $\phi:=\phi_{0} \circ E_{B}$. Then the equation $e_{B} \Lambda_{\phi}(a):=\Lambda_{\phi}\left(E_{B}(a)\right)$ defines a projection $e_{B} \in B\left(H_{\phi}\right)$ onto $\left[\Lambda_{\phi}(B)\right]$, where $[S]$ is in general the closed subspace spanned by a set $S$. We call $e_{B}$ the Jones projection of the inclusion $B \subseteq A$. The basic extension of this inclusion (by $E_{B}$ ) is the factor, denoted by $A_{1}$, acting on $H_{\phi}$ generated by $A$ and $e_{B}$. It is known that $A_{1}=J_{\phi} B^{\prime} J_{\phi}$, where $J_{\phi}$ is the modular conjugation of $\phi$. 
According to [14] (see also [13, Section 2]), there exists a faithful normal semifinite operator valued weight $\hat{E}_{B}$, called the operator valued weight dual to $E_{B}$, from $A_{1}$ to $A$. It satisfies $\hat{E}_{B}\left(e_{B}\right)=1$ [14, Lemma 3.1], so that $A e_{B} A \subseteq \mathfrak{m}_{\hat{E}_{B}}$. By [13, Proposition 2.8], the relative commutant $A_{1} \cap B^{\prime}$ admits an important decomposition into a direct sum

$$
A_{1} \cap B^{\prime}=\mathcal{A} \oplus \mathcal{B}_{1} \oplus \mathcal{B}_{2} \oplus \mathcal{C}
$$

of four subalgebras $\mathcal{A}, \mathcal{B}_{1}, \mathcal{B}_{2}$ and $\mathcal{C}$ enjoying a certain set of properties. We refer to this decomposition as the ILP decomposition of $A_{1} \cap B^{\prime}$. We say (see [13, Definition 3.7]) that the inclusion $B \subseteq A$ is discrete if $\mathcal{B}_{1}=\mathcal{B}_{2}=\mathcal{C}=\{0\}$ in the ILP decomposition (2.1). Note that $B \subseteq A$ is discrete if and only if $\left.\hat{E}_{B}\right|_{A_{1} \cap B^{\prime}}$ is semifinite.

\section{$\S 2.3$. The commensurability subrelation}

Let us assume that our discrete equivalence relation $\mathcal{R}$ is ergodic, and consider the factor $A:=W^{*}(\mathcal{R}, \omega)$ on the Hilbert space $L^{2}(\mathcal{R}, \nu)$ for some 2-cocycle $\omega$. We also consider an ergodic Borel subrelation $\mathcal{S}$ of $\mathcal{R}$ and its associated subfactor $B:=W^{*}(\mathcal{S}, \omega)$ of $A$. According to [3], the commensurability groupoid $\mathcal{C G}(B)$ of $B$ in $A$ is by definition the set of all partial isometries $v \in A$ satisfying the following two conditions:

- Both $v^{*} v$ and $v v^{*}$ belong to $B$.

- The projections $z_{v}$ and $z_{v^{*}}$ belong to $\mathfrak{m}_{\hat{E}_{B}}^{+}$, where, for an element $a \in A, z_{a}$ denotes the projection onto $\left[B a B \xi_{0}\right]$ which belongs to $A_{1} \cap B^{\prime}$.

It is shown in [3, Theorem 7.1] that the inclusion $B \subseteq A$ is discrete in the sense explained in Subsection 2.2 if and only if the subfactor generated by $\mathcal{C G}(B)$ coincides with $A$. We denote by $\operatorname{Comm}_{\mathcal{R}}(\mathcal{S})$ the Borel equivalence subrelation determined, uniquely up to a $\nu$-null set, by the intermediate subfactor $\mathcal{C G}(B)^{\prime \prime}$, and call it the commensurability subrelation of $\mathcal{S}$ in $\mathcal{R}$. Thus $\mathcal{C} \mathcal{G}(B)^{\prime \prime}=W^{*}\left(\operatorname{Comm}_{\mathcal{R}}(\mathcal{S}), \omega\right)$. We refer the readers to [2] as well for a measure-theoretical approach to this notion of commensurability.

\section{§3. The relative commutant $W^{*}\left(I_{\sigma} \times \mathcal{R}, \omega^{\sigma}\right) \cap \Pi(B)^{\prime}$}

Throughout the rest of this paper, our discrete measured equivalence relation $\mathcal{R}$ on $(X, \mathfrak{B}, \mu)$ is assumed to be ergodic, unless otherwise stated. In this section, we also fix an ergodic Borel subrelation $\mathcal{S}$ of $\mathcal{R}$. Choose choice functions $\left\{\psi_{j}\right\}_{j \in I}$ for the pair $\mathcal{S} \subseteq \mathcal{R}$ and consider the associated index cocycle $\sigma$. Here $I=\{0,1, \ldots, N\}$ $(N$ could be $\infty)$, and we assume that $\psi_{0}=\mathrm{id}_{X}$. 
We consider the factor $A:=W^{*}(\mathcal{R}, \omega)$. Write $D$ for the Cartan subalgebra of $A$ and $E_{D}$ for the faithful normal conditional expectation from $A$ to $D$. Also inside $A$, we have the subfactor $B:=W^{*}(\mathcal{S}, \omega)$ of $A$ corresponding to $\mathcal{S}$. We have a (unique) faithful normal $\theta$-invariant conditional expectation $E_{B}$ from $A$ onto $B$. As before, let $e_{B}$ be the Jones projection of $B \subseteq A$.

Denote by $I_{\sigma} \times \mathcal{R}$ the discrete measured equivalence relation on $I \times X$ defined by $((i, x),(j, y)) \in I_{\sigma} \times \mathcal{R}$ if and only if $(x, y) \in \mathcal{R}$ and $j=\sigma(y, x)(i)$. We call this equivalence relation the skew-product of $\mathcal{R}$ by $\sigma$. Note that the 2-cocycle $\omega$ on $\mathcal{R}$ can be naturally lifted to one, denoted by $\omega^{\sigma}$, on $I_{\sigma} \times \mathcal{R}$.

In [3, Theorem 4.2], it is proven that the von Neumann algebra $W^{*}\left(I_{\sigma} \times \mathcal{R}, \omega^{\sigma}\right)$ of the skew-product of $\mathcal{R}$ by $\sigma$ is $*$-isomorphic to the basic extension $A_{1}$ of the inclusion $B \subseteq A$. As in the proof of [3, Theorem 4.2], define a unitary $V_{\sigma}$ on $\ell^{2}(I) \otimes L^{2}(\mathcal{R})$ by

$$
\left\{V_{\sigma} \xi\right\}(j,(x, y)):=\xi(\sigma(y, x)(j),(x, y))
$$

Then consider the $*$-homomorphism $\Pi$ given by

$$
\Pi(a):=V_{\sigma}(1 \otimes a) V_{\sigma}^{*} \quad(a \in A)
$$

If $a=L^{\omega}(f) \in A$, then we have

$$
\{\Pi(a) \xi\}(j,(x, z))=\sum_{y \sim x} f(x, y) \xi(\sigma(y, x)(j),(y, z)) \omega(x, y, z)
$$

for any $\xi \in \ell^{2}(I) \otimes L^{2}(\mathcal{R})$. Then $W^{*}\left(I_{\sigma} \times \mathcal{R}, \omega^{\sigma}\right)$ is generated by $\Pi(A)$ and $\ell^{\infty}(I) \otimes \mathbb{C}$. For any $j \in I$, define a function $\delta_{j}$ on $I$ by $\delta_{j}(i):=\delta_{i, j}$. According to 3. Theorem 4.2], there exists a $*$-isomorphism $\Phi$ from $A_{1}$ onto $W^{*}\left(I_{\sigma} \times \mathcal{R}, \omega^{\sigma}\right)$ satisfying $\Phi(a)=\Pi(a)$ (for all $a \in A$ ) and $\Phi\left(e_{B}\right)=\delta_{0} \otimes 1$.

Before we proceed to the next proposition, note that $W^{*}\left(I_{\sigma} \times \mathcal{R}, \omega^{\sigma}\right)$ is contained in $B\left(\ell^{2}(I)\right) \otimes A$. Hence the restriction of $\operatorname{id}_{B\left(\ell^{2}(I)\right)} \otimes E_{D}$ to $W^{*}\left(I_{\sigma} \times \mathcal{R}, \omega^{\sigma}\right)$ makes sense.

Proposition 3.1. The restriction $E^{\sigma}:=\left.\left(\mathrm{id} \otimes E_{D}\right)\right|_{W^{*}\left(I_{\sigma} \times \mathcal{R}, \omega^{\sigma}\right)}$ is the unique faithful normal conditional expectation from $W^{*}\left(I_{\sigma} \times \mathcal{R}, \omega^{\sigma}\right)$ onto the Cartan subalgebra $\ell^{\infty}(I) \otimes D$.

Proof. It is obvious that $\left.E^{\sigma}\right|_{\ell^{\infty}(I) \otimes D}=\mathrm{id}$.

Let $f, g \in \mathfrak{A}_{I}$. Note that $\chi_{\mathcal{D}} a \chi_{\mathcal{D}}=E_{D}(a) \chi_{\mathcal{D}}$ for all $a \in A$. It follows that $\left(1 \otimes \chi_{\mathcal{D}}\right) T\left(1 \otimes \chi_{\mathcal{D}}\right)=E^{\sigma}(T)\left(1 \otimes \chi_{\mathcal{D}}\right)$ for any $T \in W^{*}\left(I_{\sigma} \times \mathcal{R}, \omega^{\sigma}\right)$. For any $\xi \in \ell^{2}(I) \otimes L^{2}(\mathcal{R})$ and $f \in \mathfrak{A}_{I}$, we have 


$$
\begin{aligned}
& \left\{\left(1 \otimes \chi_{\mathcal{D}}\right) \Pi\left(L^{\omega}(f)\right)\left(\delta_{0} \otimes 1\right) \Pi\left(L^{\omega}(g)\right)\left(1 \otimes \chi_{\mathcal{D}}\right) \xi\right\}(j,(x, z)) \\
& =\delta_{x, z}\left\{\Pi\left(L^{\omega}(f)\right)\left(\delta_{0} \otimes 1\right) \Pi\left(L^{\omega}(g)\right)\left(1 \otimes \chi_{\mathcal{D}}\right) \xi\right\}(j,(x, x)) \\
& =\delta_{x, z} \sum_{y \sim x} f(x, y)\left\{\left(\delta_{0} \otimes 1\right) \Pi\left(L^{\omega}(g)\right)\left(1 \otimes \chi_{\mathcal{D}}\right) \xi\right\}(\sigma(y, x)(j),(y, x)) \\
& =\delta_{x, z} \sum_{y \sim x} \delta_{0, \sigma(y, x)(j)} f(x, y)\left\{\Pi\left(L^{\omega}(g)\right)\left(1 \otimes \chi_{\mathcal{D}}\right) \xi\right\}(0,(y, x)) \\
& =\delta_{x, z} \sum_{y \sim x} \delta_{0, \sigma(y, x)(j)} f(x, y)\left(\sum_{w \sim x} g(y, w)\left\{\left(1 \otimes \chi_{\mathcal{D}}\right) \xi\right\}(\sigma(w, y)(0),(w, x)) \omega(y, w, x)\right) \\
& =\delta_{x, z} \sum_{y \sim x} \delta_{0, \sigma(y, x)(j)} f(x, y) g(y, x) \xi(\sigma(x, y)(0),(x, x)) \\
& =\delta_{x, z} \sum_{y \sim x} \delta_{j, \sigma(x, y)(0)} f(x, y) g(y, x) \xi(j,(x, x)) \\
& =\sum_{y \sim x} \delta_{\sigma(x, y)(0)}(j) f(x, y) g(y, x)\left\{\left(1 \otimes \chi_{\mathcal{D}}\right) \xi\right\}(j,(x, z)) .
\end{aligned}
$$

So, if we define a bounded function $P_{f, g}$ on $I \times X$ by

$$
P_{f, g}(j, x):=\sum_{y \sim x} \delta_{\sigma(x, y)(0)}(j) f(x, y) g(y, x),
$$

then we obtain $\left(1 \otimes \chi_{\mathcal{D}}\right) \Pi\left(L^{\omega}(f)\right)\left(\delta_{0} \otimes 1\right) \Pi\left(L^{\omega}(g)\right)\left(1 \otimes \chi_{\mathcal{D}}\right)=P_{f, g}\left(1 \otimes \chi_{\mathcal{D}}\right)$. Hence

$$
E^{\sigma}\left(\Pi\left(L^{\omega}(f)\right)\left(\delta_{0} \otimes 1\right) \Pi\left(L^{\omega}(g)\right)\right)\left(1 \otimes \chi_{\mathcal{D}}\right)=P_{f, g}\left(1 \otimes \chi_{\mathcal{D}}\right) .
$$

Since $\overline{A^{\prime} \chi_{\mathcal{D}}}=L^{2}(\mathcal{R})$, we find that $E^{\sigma}\left(\Pi\left(L^{\omega}(f)\right)\left(\delta_{0} \otimes 1\right) \Pi\left(L^{\omega}(g)\right)\right)=P_{f, g}$. Hence the image of the linear span of the set $\left\{\Pi\left(L^{\omega}(f)\right)\left(\delta_{0} \otimes 1\right) \Pi\left(L^{\omega}(g)\right): f, g \in \mathfrak{A}_{I}\right\}$ under the map $E^{\sigma}$ is contained in $\ell^{\infty}(I) \otimes D$. Therefore, the range of $E^{\sigma}$ equals $\ell^{\infty}(I) \otimes D$. Since $\ell^{\infty}(I) \otimes D$ is maximal abelian, $E^{\sigma}$ is the unique faithful normal conditional expectation from $W^{*}\left(I_{\sigma} \times \mathcal{R}, \omega^{\sigma}\right)$ onto $\ell^{\infty}(I) \otimes D$.

Theorem 3.2. The relative commutant $W^{*}\left(I_{\sigma} \times \mathcal{R}, \omega^{\sigma}\right) \cap \Pi(B)^{\prime}$ consists precisely of all the functions $F$ in the Cartan subalgebra $\ell^{\infty}(I) \otimes D$ satisfying

$$
F(j, x)=F(\sigma(y, x)(j), y) \quad(\text { a.e. }(x, y) \in \mathcal{S}, \forall j \in I) .
$$

Proof. First note that $\Pi(d)=1 \otimes d$ for all $d \in D$. Hence

$$
W^{*}\left(I_{\sigma} \times \mathcal{R}, \omega^{\sigma}\right) \cap \Pi(B)^{\prime} \subseteq\left(B\left(\ell^{2}(I)\right) \otimes A\right) \cap(\mathbb{C} \otimes D)^{\prime}=B\left(\ell^{2}(I)\right) \otimes D .
$$

From this and Proposition 3.1. we have, for any $T \in W^{*}\left(I_{\sigma} \times \mathcal{R}, \omega^{\sigma}\right) \cap \Pi(B)^{\prime}$,

$$
T=\left(\operatorname{id}_{B\left(\ell^{2}(I)\right)} \otimes E_{D}\right)(T)=E^{\sigma}(T) \in \ell^{\infty}(I) \otimes D .
$$


Thus $T$ is the multiplication operator by a function $F$ in $\ell^{\infty}(I) \otimes D$. We also have $\Pi\left(L^{\omega}(f)\right) F=F \Pi\left(L^{\omega}(f)\right)$ for any $f \in \mathfrak{A}_{I}$ with $\{(x, y) \in \mathcal{R}: f(x, y) \neq 0\}$ $\subseteq \mathcal{S}$. But, thanks to $(3.1)$, this identity is easily seen to be equivalent to the condition 3.2 . 3.2 .

We denote by $L^{\infty}(I \times X)^{\mathcal{S}}$ the set of all functions $F$ in $L^{\infty}(I \times X)$ satisfying

\section{$\S 4$. The dual operator valued weight of $E_{B}$}

We retain the notation introduced in the preceding section.

As in Section 4 of [3], we set $\mathcal{R}_{j}:=\{(x, y) \in \mathcal{R}: \sigma(x, y)(0)=j\}$ for each $j \in I$. By a direct computation, we get

$$
\left\{V_{\sigma}^{*}\left(\delta_{0} \otimes 1\right) V_{\sigma} \xi\right\}(j,(x, y))= \begin{cases}\xi(j,(x, y)) & \text { if }(x, y) \in \mathcal{R}_{j}^{-1} \\ 0 & \text { otherwise. }\end{cases}
$$

This clearly implies

$$
V_{\sigma}^{*}\left(\delta_{0} \otimes 1\right) V_{\sigma}=\sum_{j \in I} \delta_{j} \otimes \chi_{\mathcal{R}_{j}^{-1}}
$$

where $\mathcal{R}_{j}^{-1}:=\left\{(x, y) \in \mathcal{R}:(y, x) \in \mathcal{R}_{j}\right\}$. Let $\mathrm{Tr}$ denote the usual trace on $B\left(\ell^{2}(I)\right)$, that is, $\operatorname{Tr}(X):=\sum_{j \in I}\left(X \delta_{j} \mid \delta_{j}\right)\left(X \in B\left(\ell^{2}(I)\right)_{+}\right)$. Then, by (4.1), we have, for any $a \in A$,

$$
\begin{aligned}
& \left(\operatorname{Tr} \otimes \operatorname{id}_{B\left(L^{2}(\mathcal{R})\right)}\right)\left(V_{\sigma}^{*} \Pi\left(a^{*}\right)\left(\delta_{0} \otimes 1\right) \Pi(a) V_{\sigma}\right) \\
& \quad=\left(\operatorname{Tr} \otimes \operatorname{id}_{B\left(L^{2}(\mathcal{R})\right)}\right)\left(\left(1 \otimes a^{*}\right) V_{\sigma}^{*}\left(\delta_{0} \otimes 1\right) V_{\sigma}(1 \otimes a)\right) \\
& \quad=\sum_{j \in I} a^{*}\left(\operatorname{Tr} \otimes \operatorname{id}_{B\left(L^{2}(\mathcal{R})\right)}\right)\left(\delta_{j} \otimes \chi_{\mathcal{R}_{j}^{-1}}\right) a=\sum_{j \in I} a^{*} \chi_{\mathcal{R}_{j}^{-1}} a=a^{*} a .
\end{aligned}
$$

This shows that $V_{\sigma}^{*} \Pi\left(a^{*}\right)\left(\delta_{0} \otimes 1\right) \Pi(a) V_{\sigma}$ belongs to $\mathfrak{m}_{\mathrm{Tr} \otimes \mathrm{id}}^{+}$, which in turn implies that

$$
\operatorname{span}\left\{V_{\sigma}^{*} \Pi(A)\left(\delta_{0} \otimes 1\right) \Pi(A) V_{\sigma}\right\}
$$

is contained in $\mathfrak{m}_{\operatorname{Tr} \otimes \mathrm{id}}$. By the computation made above, we have

$$
\left(\operatorname{Tr} \otimes \operatorname{id}_{B\left(L^{2}(\mathcal{R})\right)}\right)\left(\operatorname{span}\left\{V_{\sigma}^{*} \Pi(A)\left(\delta_{0} \otimes 1\right) \Pi(A) V_{\sigma}\right\}\right) \subseteq A .
$$

Lemma 4.1. For any $X \in W^{*}\left(I_{\sigma} \times \mathcal{R}, \omega^{\sigma}\right)_{+},\left(\operatorname{Tr} \otimes \operatorname{id}_{B\left(L^{2}(\mathcal{R})\right)}\right)\left(V_{\sigma}^{*} X V_{\sigma}\right)$ belongs to the extended positive part $\widetilde{A_{+}}$of $A$.

Proof. Let $X \in W^{*}\left(I_{\sigma} \times \mathcal{R}, \omega^{\sigma}\right)_{+}$. Since $\operatorname{span}\left\{V_{\sigma}^{*} \Pi(A)\left(\delta_{0} \otimes 1\right) \Pi(A) V_{\sigma}\right\}$ is a $\sigma$ strongly* dense two-sided ideal of $W^{*}\left(I_{\sigma} \times \mathcal{R}, \omega^{\sigma}\right)$, it follows from [21, Chapter II, 
Proposition 3.13] that there exists an increasing net $\left\{X_{\lambda}\right\}_{\lambda \in \Lambda}$ of positive elements in $\operatorname{span}\left\{V_{\sigma}^{*} \Pi(A)\left(\delta_{0} \otimes 1\right) \Pi(A) V_{\sigma}\right\}$ such that $X$ is the $\sigma$-strong limit of $\left\{X_{\lambda}\right\}$. Let $u \in A^{\prime}$ be any unitary and $\psi \in B\left(L^{2}(\mathcal{R})\right)_{*}^{+}$. Then, by 4.2 , we have

$$
\begin{aligned}
\left(\operatorname{Tr} \otimes \operatorname{id}_{B\left(L^{2}(\mathcal{R})\right)}\right) & \left(V_{\sigma}^{*} X V_{\sigma}\right)\left(u \psi u^{*}\right)=\left(\operatorname{Tr} \otimes u \psi u^{*}\right)\left(V_{\sigma}^{*} X V_{\sigma}\right) \\
& =\lim _{\lambda \in \Lambda}\left(\operatorname{Tr} \otimes u \psi u^{*}\right)\left(V_{\sigma}^{*} X_{\lambda} V_{\sigma}\right)=\lim _{\lambda \in \Lambda}(\operatorname{Tr} \otimes \psi)\left(V_{\sigma}^{*} X_{\lambda} V_{\sigma}\right) \\
& =(\operatorname{Tr} \otimes \psi)\left(V_{\sigma}^{*} X V_{\sigma}\right)=\left(\operatorname{Tr} \otimes \operatorname{id}_{B\left(L^{2}(\mathcal{R})\right)}\right)\left(V_{\sigma}^{*} X V_{\sigma}\right)(\psi) .
\end{aligned}
$$

Hence $\left(\operatorname{Tr} \otimes \operatorname{id}_{B\left(L^{2}(\mathcal{R})\right)}\right)\left(V_{\sigma}^{*} X V_{\sigma}\right)$ falls in $\widetilde{A_{+}}$.

For the next theorem, note that $\Pi\left(\left(\operatorname{Tr} \otimes \operatorname{id}_{B\left(L^{2}(\mathcal{R})\right)}\right)\left(V_{\sigma}^{*} X V_{\sigma}\right)\right)$ belongs to the extended positive part $\widetilde{\Pi(A)_{+}}$of $\Pi(A)$ for any $X \in W^{*}\left(I_{\sigma} \times \mathcal{R}, \omega^{\sigma}\right)_{+}$.

Theorem 4.2. The map

$$
X \in W^{*}\left(I_{\sigma} \times \mathcal{R}, \omega^{\sigma}\right)_{+} \mapsto \Pi\left(\left(\operatorname{Tr} \otimes \operatorname{id}_{B\left(L^{2}(\mathcal{R})\right)}\right)\left(V_{\sigma}^{*} X V_{\sigma}\right)\right)
$$

defines a unique faithful normal semifinite operator valued weight $T_{B}$ from $W^{*}\left(I_{\sigma} \times\right.$ $\left.\mathcal{R}, \omega^{\sigma}\right)$ to $\Pi(A)$ satisfying $T_{B}\left(\delta_{0} \otimes 1\right)=1$.

Proof. It is easy to see that $T_{B}: W^{*}\left(I_{\sigma} \times \mathcal{R}, \omega^{\sigma}\right)_{+} \rightarrow \widetilde{\Pi(A)_{+}}$satisfies

$$
\begin{aligned}
T_{B}(X+Y) & =T_{B}(X)+T_{B}(Y), \\
T_{B}(c X) & =c T_{B}(X) \quad\left(\forall X, Y \in W^{*}\left(I_{\sigma} \times \mathcal{R}, \omega^{\sigma}\right)_{+}, c \in[0, \infty)\right) .
\end{aligned}
$$

Let $a \in A$ and $X \in W^{*}\left(I_{\sigma} \times \mathcal{R}, \omega^{\sigma}\right)_{+}$. Then

$$
\begin{aligned}
T_{B}\left(\Pi(a)^{*} X \Pi(a)\right) & =\Pi\left(\left(\operatorname{Tr} \otimes \operatorname{id}_{B\left(L^{2}(\mathcal{R})\right)}\right)\left(V_{\sigma}^{*} \Pi(a)^{*} X \Pi(a) V_{\sigma}\right)\right) \\
& =\Pi\left(\left(\operatorname{Tr} \otimes \operatorname{id}_{B\left(L^{2}(\mathcal{R})\right)}\right)\left(\left(1 \otimes a^{*}\right) V_{\sigma}^{*} X V_{\sigma}(1 \otimes a)\right)\right. \\
& =\Pi\left(a^{*}\left(\operatorname{Tr} \otimes \operatorname{id}_{B\left(L^{2}(\mathcal{R})\right)}\right)\left(V_{\sigma}^{*} X V_{\sigma}\right) a\right)=\Pi(a)^{*} T_{B}(X) \Pi(a) .
\end{aligned}
$$

Thus $T_{B}$ is an operator valued weight from $W^{*}\left(I_{\sigma} \times \mathcal{R}, \omega^{\sigma}\right)$ to $\Pi(A)$. The faithfulness and normality of $T_{B}$ are immediate. Thanks to $(4.2), T_{B}$ is semifinite.

Since $\left(\Pi(A) \subseteq W^{*}\left(I_{\sigma} \times \mathcal{R}, \omega^{\sigma}\right)\right)$ is isomorphic to $\left(A \subseteq A_{1}\right)$ through the isomorphism $\Phi$, we have $W^{*}\left(I_{\sigma} \times \mathcal{R}, \omega^{\sigma}\right) \cap \Pi(A)^{\prime}=\mathbb{C}$. It then follows from [5, Proposition 11.1] that any other faithful normal semifinite operator valued weight from $W^{*}\left(I_{\sigma} \times \mathcal{R}, \omega^{\sigma}\right)$ to $\Pi(A)$ is proportional to $T_{B}$. Hence $T_{B}$ is uniquely determined by the condition $T_{B}\left(\delta_{0} \otimes 1\right)=1$.

Remark. Recall the isomorphism $\Phi: A_{1} \rightarrow W^{*}\left(I_{\sigma} \times \mathcal{R}, \omega^{\sigma}\right)$. The map $\Phi \circ \widehat{E}_{B} \circ \Phi^{-1}$ is also a faithful normal semifinite operator valued weight from $W^{*}\left(I_{\sigma} \times \mathcal{R}, \omega^{\sigma}\right)$ to $\Pi(A)$. Since $\Phi\left(e_{B}\right)=\delta_{0} \otimes 1$, the above operator valued weight is exactly $T_{B}$, 
due to Theorem 4.2. Hence we shall often call $T_{B}$ the dual operator valued weight of $E_{B}$.

Corollary 4.3. The restriction of $T_{B}$ to the relative commutant $W^{*}\left(I_{\sigma} \times \mathcal{R}, \omega^{\sigma}\right)$ $\cap \Pi(B)^{\prime}=L^{\infty}(I \times X)^{\mathcal{S}}$ is given by

$$
F \in\left(L^{\infty}(I \times X)^{\mathcal{S}}\right)_{+} \mapsto\left(\int_{X} \sum_{j \in I} F(j, x) d m(x)\right) \cdot 1
$$

for any probability measure $m$ on $X$ equivalent to $\mu$.

Proof. Let $F \in\left(L^{\infty}(I \times X)^{\mathcal{S}}\right)_{+}$. Then $V_{\sigma}^{*} F V_{\sigma}$ is the function on $I \times \mathcal{R}$ that assigns $F(\sigma(x, y)(j), x)$ to $(j,(x, y))$. Thus we have

$$
\left(\operatorname{Tr} \otimes \operatorname{id}_{B\left(L^{2}(\mathcal{R})\right)}\right)\left(V_{\sigma}^{*} F V_{\sigma}\right)=\sum_{j \in I} F(j, \cdot) \in \widetilde{D_{+}} .
$$

Since $F$ belongs to $L^{\infty}(I \times X)^{\mathcal{S}}$, the function $x \in X \mapsto \sum_{j \in I} F(j, x)$ is $\mathcal{S}$-invariant up to a null set. Because $\mathcal{S}$ is ergodic, this function is constant. Hence $T_{B}(F)$ equals $\left(\int_{X} \sum_{j \in I} F(j, x) d m(x)\right) \cdot 1$.

\section{$\S 5$. The relative commutant $\left(B\left(\ell^{2}(I)\right) \otimes A\right) \cap \Pi(A)^{\prime}$}

We shall retain the notation introduced in the previous sections.

Let $\widetilde{\mathfrak{A}}_{I}$ be a counterpart of the left Hilbert algebra $\mathfrak{A}_{I}$, constructed from the discrete equivalence relation $I_{\sigma} \times \mathcal{R}$. Denote by $L_{1}^{\omega}(F)$ the left multiplication (convolution) of $F \in \widetilde{\mathfrak{A}}_{I}$ :

$\left\{L_{1}^{\omega}(F) \xi\right\}(j,(x, y)):=\sum_{z \sim x} F(j,(x, z)) \xi(\sigma(z, x)(j),(z, y)) \omega(x, z, y)\left(\xi \in L^{2}(I \times \mathcal{R})\right)$,

where we always think of any $F$ in $\widetilde{\mathfrak{A}}_{I}$ as a function on $I \times \mathcal{R}$ by identifying each element $((j, x),(\sigma(y, x)(j), y)) \in I_{\sigma} \times \mathcal{R}$ with $(j,(x, y)) \in I \times \mathcal{R}$.

The modular operator of $\widetilde{\mathfrak{A}}_{I}$ (= the module of the equivalence relation $I_{\sigma} \times \mathcal{R}$ ) is $1 \otimes \delta$, and its modular conjugation $\tilde{J}$ is given by

$$
\{\tilde{J} \xi\}(j,(x, y))=\delta(y, x)^{1 / 2} \overline{\xi(\sigma(y, x)(j),(y, x))} \quad\left(\xi \in L^{2}(I \times \mathcal{R})\right) .
$$

Our first task is to locate the basic extension of the inclusion $\Pi(A) \subseteq$ $W^{*}\left(I_{\sigma} \times \mathcal{R}, \omega^{\sigma}\right)$ on the Hilbert space $\ell^{2}(I) \otimes L^{2}(\mathcal{R})$. It is by definition $\tilde{J} \Pi(A)^{\prime} \overline{\tilde{J}}$. Observe that

$$
\begin{aligned}
\left\{\tilde{J} V_{\sigma} \xi\right\}(j,(x, y)) & =\delta(y, x)^{1 / 2} \overline{\left\{V_{\sigma} \xi\right\}(\sigma(y, x)(j),(y, x))} \\
& =\delta(y, x)^{1 / 2} \overline{\xi(j,(y, x))}=\left\{\left(J_{0} \otimes J\right) \xi\right\}(j,(x, y)),
\end{aligned}
$$


where $J_{0}: \ell^{2}(I) \rightarrow \ell^{2}(I)$ is given by $\left\{J_{0} f\right\}(j)=\overline{f(j)}$. Hence

$$
\begin{aligned}
\tilde{J} \Pi(A)^{\prime} \tilde{J} & =\tilde{J} V_{\sigma}\left(B\left(\ell^{2}(I)\right) \otimes A^{\prime}\right) V_{\sigma}^{*} \tilde{J}=\left(J_{0} \otimes J\right)\left(B\left(\ell^{2}(I)\right) \otimes A^{\prime}\right)\left(J_{0} \otimes J\right) \\
& =J_{0} B\left(\ell^{2}(I)\right) J_{0} \otimes J A^{\prime} J=B\left(\ell^{2}(I)\right) \otimes A .
\end{aligned}
$$

Therefore, the basic extension of the inclusion $\Pi(A) \subseteq W^{*}\left(I_{\sigma} \times \mathcal{R}, \omega^{\sigma}\right)$ on the Hilbert space $\ell^{2}(I) \otimes L^{2}(\mathcal{R})$ is $B\left(\ell^{2}(I)\right) \otimes A$.

Our next goal is to locate the relative commutant $\left(B\left(\ell^{2}(I)\right) \otimes A\right) \cap \Pi(A)^{\prime}$. Since $D \subseteq A$ and $\Pi(d)=1 \otimes d$ for any $d \in D$, it follows that

$$
\begin{aligned}
\left(B\left(\ell^{2}(I)\right) \otimes A\right) \cap \Pi(A)^{\prime} & \subseteq\left(B\left(\ell^{2}(I)\right) \otimes A\right) \cap(\mathbb{C} \otimes D)^{\prime} \\
& =B\left(\ell^{2}(I)\right) \otimes\left(A \cap D^{\prime}\right)=B\left(\ell^{2}(I)\right) \otimes D .
\end{aligned}
$$

For each $j \in I$, define operators $S_{j}: \ell^{2}(I) \otimes L^{2}(\mathcal{R}) \rightarrow L^{2}(\mathcal{R})$ and $T_{j}: L^{2}(\mathcal{R}) \rightarrow$ $\ell^{2}(I) \otimes L^{2}(\mathcal{R})$ by

$$
S_{j}\left(\sum_{i \in I} \delta_{i} \otimes \xi_{i}\right):=\xi_{j}, \quad T_{j} \xi:=\delta_{j} \otimes \xi
$$

For an operator $Y \in B\left(\ell^{2}(I) \otimes L^{2}(\mathcal{R})\right)$, let $\left[Y_{i, j}\right]_{i, j \in I}$ stand for the matrix representation of $Y$, that is, $Y_{i, j}:=S_{i} Y T_{j} \in B\left(L^{2}(\mathcal{R})\right)$.

Let $R \in\left(B\left(\ell^{2}(I)\right) \otimes A\right) \cap \Pi(A)^{\prime}$. By the above computation, each $R_{i, j}$ belongs to $D$. For any $f \in \mathfrak{A}_{I}$, a direct calculation shows that

$$
\left\{\Pi\left(L^{\omega}(f)\right)_{i, j} \xi\right\}(x, y)=\sum_{z \sim x} f(x, z) \delta_{\sigma(z, x)(i), j} \xi(z, y) \omega(x, z, y) \quad\left(\xi \in L^{2}(\mathcal{R})\right) .
$$

Hence

$$
\begin{aligned}
& \left(R \Pi\left(L^{\omega}(f)\right)\right)_{i, j}(x, y)=\sum_{z \sim x} f(x, z)\left(\sum_{k \in I} R_{i, k}(x) \delta_{j, \sigma(z, x)(k)}\right) \xi(z, y) \omega(x, z, y), \\
& \left(\Pi\left(L^{\omega}(f)\right) R\right)_{i, j}(x, y)=\sum_{z \sim x} f(x, z)\left(\sum_{k \in I} R_{k, j}(z) \delta_{k, \sigma(z, x)(i)}\right) \xi(z, y) \omega(x, z, y)
\end{aligned}
$$

for $\xi \in L^{2}(\mathcal{R})$. Since $R \Pi\left(L^{\omega}(f)\right)=\Pi\left(L^{\omega}(f)\right) R$, it easily follows that

$$
\left.R_{i, \sigma(x, y)(j)}(x)=R_{\sigma(y, x)(i), j}(y) \quad \text { (a.e. }(x, y) \in \mathcal{R}\right) .
$$

Let us summarize the discussion made so far in the next theorem.

Theorem 5.1. Every element of $\left(B\left(\ell^{2}(I)\right) \otimes A\right) \cap \Pi(A)^{\prime}$ is regarded as a bounded Borel $B\left(\ell^{2}(I)\right)$-valued function $R$ on $X$ that satisfies [5.1], where $R_{i, j}(x):=$ $\left(R(x) \delta_{j} \mid \delta_{i}\right)$ for any $x \in X$ and $i, j \in I$. 
Remark. Theorem 5.1 may be obtained by an argument slightly different from the one made above, as we explain below. Note first that, with $\mathcal{R}^{x}:=\{(x, y) \in \mathcal{R}$ : $y \in \mathcal{R}(x)\}$ for any $x \in X$, the map $f \in \mathfrak{A}_{I} \mapsto \Pi\left(L^{\omega}(f)\right)$ is the "integrated form" of the groupoid representation $\Lambda$ of $\mathcal{R}$ on the Hilbert bundle $\left\{\ell^{2}(I) \otimes \ell^{2}\left(\mathcal{R}^{x}\right)\right\}_{x \in X}$ given by

$$
\Lambda(x, y)=\lambda^{\sigma}(x, y) \otimes \lambda^{\omega}(x, y) \quad((x, y) \in \mathcal{R})
$$

where $\lambda^{\sigma}(x, y): \ell^{2}(I) \rightarrow \ell^{2}(I)$ is defined by $\left\{\lambda^{\sigma}(x, y) g\right\}(j):=g(\sigma(y, x)(j))$, and $\lambda^{\omega}$ denotes the $\omega$-twisted regular representation of $\mathcal{R}$ (cf. 11, Section 3]). If $R \in$ $\left(B\left(\ell^{2}(I)\right) \otimes A\right) \cap \Pi(A)^{\prime}$, then $R$ is a measurable field of operators $\{R(x)\}_{x \in X}$ with $R(x) \in B\left(\ell^{2}(I) \otimes \ell^{2}\left(\mathcal{R}^{x}\right)\right)$ satisfying

$$
R(x) \Lambda(x, y)=\Lambda(x, y) R(y) \quad(\text { a.e. }(x, y) \in \mathcal{R}) .
$$

Since $R \in B\left(\ell^{2}(I)\right) \otimes D$, we may assume from the outset that $R(x)$ has the form $R(x)=R^{1}(x) \otimes 1$ for all $x \in X$, where $R^{1}(x) \in B\left(\ell^{2}(I)\right)$. In this case, (5.2) is equivalent to

$$
R^{1}(x) \lambda^{\sigma}(x, y)=\lambda^{\sigma}(x, y) R^{1}(y) \quad(\text { a.e. }(x, y)) .
$$

It is easy to check that the unitary $\lambda^{\sigma}(x, y) \in B\left(\ell^{2}(I)\right)$ has matrix representation $\left[\delta_{i, \sigma(x, y)(j)}\right]_{i, j \in I}$ with respect to the orthonormal basis $\left\{\delta_{j}\right\}_{j \in I}$. Hence, if $\left[R_{i, j}^{1}(x)\right]_{i, j \in I}$ is the matrix representation of $R^{1}(x)$ with respect to the same basis, (5.3) can be written in the form

$$
R_{i, \sigma(x, y)(j)}^{1}(x)=R_{\sigma(y, x)(i), j}^{1}(y) \quad(\text { a.e. }(x, y) \in \mathcal{R}) .
$$

Thus we obtain the assertion of Theorem 5.1

\section{§6. The isomorphism between $A_{1} \cap B^{\prime}$ and $W^{*}\left(I_{\sigma} \times \mathcal{R}, \omega^{\sigma}\right) \cap \Pi(B)^{\prime}$}

In this section, we examine the restriction of the $*$-isomorphism $\Phi$ to $A_{1} \cap B^{\prime}$.

Lemma 6.1. We have

$$
V_{\sigma}^{*}\left(\delta_{j} \otimes 1\right) V_{\sigma}=\sum_{k \in I} \delta_{k} \otimes \chi_{\mathcal{T}_{k, j}}
$$

where $\mathcal{T}_{k, j}:=\{(x, y) \in \mathcal{R}: \sigma(x, y)(k)=j\}$.

Proof. Let $S_{j}: \ell^{2}(I) \otimes L^{2}(\mathcal{R}) \rightarrow L^{2}(\mathcal{R})$ and $T_{j}: L^{2}(\mathcal{R}) \rightarrow \ell^{2}(I) \otimes L^{2}(\mathcal{R})$ be the operators defined in the preceding section. Then, for any $\xi \in L^{2}(\mathcal{R})$, we have 


$$
\begin{aligned}
\left\{S_{i} V_{\sigma}^{*}\left(\delta_{j} \otimes 1\right) V_{\sigma} T_{k} \xi\right\}(x, y) & =\left\{V_{\sigma}^{*}\left(\delta_{j} \otimes 1\right) V_{\sigma}\left(\delta_{k} \otimes \xi\right)\right\}(i,(x, y)) \\
& =\left\{\left(\delta_{j} \otimes 1\right) V_{\sigma}\left(\delta_{k} \otimes \xi\right)\right\}(\sigma(x, y)(i),(x, y)) \\
& =\delta_{j}(\sigma(x, y)(i))\left\{V_{\sigma}\left(\delta_{k} \otimes \xi\right)\right\}(\sigma(x, y)(i),(x, y)) \\
& =\delta_{j}(\sigma(x, y)(i)) \delta_{k}(i) \xi(x, y)=\delta_{i, k} \chi_{\mathcal{T}_{k, j}}(x, y) \xi(x, y) .
\end{aligned}
$$

This shows that $V_{\sigma}^{*}\left(\delta_{j} \otimes 1\right) V_{\sigma}$ has matrix representation $\left[\delta_{i, k} \chi_{\mathcal{T}_{k, j}}\right]_{i, k \in I}$.

Lemma 6.2. With the notation in Lemma6.1, we have

$$
T_{B}\left(\left(\delta_{j} \otimes 1\right) \Pi(a)\left(\delta_{j^{\prime}} \otimes 1\right)\right)=\Pi\left(\sum_{k \in I} \chi_{\mathcal{T}_{k, j}} a \chi_{\mathcal{T}_{k, j^{\prime}}}\right)
$$

for any $a \in A$ and $j, j^{\prime} \in I$. In particular, if $a=L^{\omega}(f)$ for some $f \in \mathfrak{A}_{I}$, then

$$
T_{B}\left(\left(\delta_{j} \otimes 1\right) \Pi\left(L^{\omega}(f)\right)\left(\delta_{j^{\prime}} \otimes 1\right)\right)=\Pi\left(L^{\omega}\left(\chi_{\mathcal{T}_{j^{\prime}, j}} f\right)\right) .
$$

Proof. By the proof of [3, Theorem 4.2, Corollary 4.6], we have $\Phi\left(\delta_{j} \otimes 1\right)=\chi_{\mathcal{R}_{j}}$ and $\widehat{E}_{B}\left(\chi_{\mathcal{R}_{j}}\right)=1$ for all $j \in I$. Since $\Phi \circ \widehat{E}_{B} \circ \Phi^{-1}=T_{B}$, we obtain $T_{B}\left(\delta_{j} \otimes 1\right)=1$ for any $j \in I$. Hence $\left(\delta_{j} \otimes 1\right) \Pi(a)\left(\delta_{j^{\prime}} \otimes 1\right)$ belongs to $\mathfrak{m}_{T_{B}}$ for any $a \in A$ and $j, j^{\prime} \in I$. Thanks to Lemma 6.1, we have

$$
\begin{aligned}
(\operatorname{Tr} \otimes \mathrm{id})\left(V_{\sigma}^{*}\left(\delta_{j} \otimes 1\right)\right. & \left.\Pi(a)\left(\delta_{j^{\prime}} \otimes 1\right) V_{\sigma}\right) \\
= & (\operatorname{Tr} \otimes \mathrm{id})\left(V_{\sigma}^{*}\left(\delta_{j} \otimes 1\right) V_{\sigma}(1 \otimes a) V_{\sigma}^{*}\left(\delta_{j^{\prime}} \otimes 1\right) V_{\sigma}\right) \\
= & (\operatorname{Tr} \otimes \mathrm{id})\left(\sum_{k \in I} \delta_{k} \otimes \chi_{\mathcal{T}_{k, j}} a \chi_{\mathcal{T}_{k, j^{\prime}}}\right)=\sum_{k \in I} \chi_{\mathcal{T}_{k, j}} a \chi_{\mathcal{T}_{k, j^{\prime}}} .
\end{aligned}
$$

Thus we obtain the first asserted identity. If $a=L^{\omega}(f)$ for some $f \in \mathfrak{A}_{I}$, then an easy computation shows that $\sum_{k \in I} \chi_{\mathcal{T}_{k, j}} L^{\omega}(f) \chi_{\mathcal{T}_{k, j^{\prime}}} \xi_{0}=\chi_{\mathcal{T}_{j^{\prime}, j}} f$, which yields the second assertion.

Lemma 6.3. We have

$$
\left(\Pi\left(L^{\omega}(f)\right)\left(\delta_{j} \otimes g\right) \mid \delta_{j^{\prime}} \otimes h\right)=\left(L^{\omega}\left(\chi_{\mathcal{T}_{j, j^{\prime}}} f\right) g \mid h\right)
$$

for any $f \in \mathfrak{A}_{I}, g, h \in L^{2}(\mathcal{R})$ and $j, j^{\prime} \in I$.

Proof. The asserted identity can be easily verified by a straightforward computation using (3.1).

Lemma 6.4. Let $\rho:=\theta \circ \Pi^{-1} \circ T_{B}$. Then the Hilbert space $H_{\rho}$ obtained from the weight $\rho$ is identified with $\ell^{2}(I) \otimes L^{2}(\mathcal{R})$ through the unitary $\mathcal{U}$ characterized by

$$
\mathcal{U} \Pi\left(L^{\omega}(f)\right) \Lambda_{\rho}\left(\left(\delta_{j} \otimes 1\right) \Pi\left(L^{\omega}(g)\right)\right)=\Pi\left(L^{\omega}(f)\right)\left(\delta_{j} \otimes g\right) \quad\left(f, g \in \mathfrak{A}_{I}, j \in I\right) .
$$


Proof. By [13. Lemma 2.1] (see the proof of [13, Proposition 2.2] also), the image $\Lambda_{\rho}\left(\Pi\left(L^{\omega}\left(\mathfrak{A}_{I}\right)\right)\left(\delta_{0} \otimes 1\right) \Pi\left(L^{\omega}\left(\mathfrak{A}_{I}\right)\right)\right)$ is dense in $H_{\rho}$. In particular, the linear span of the vectors of the form $\Lambda_{\rho}\left(\Pi\left(L^{\omega}(f)\right)\left(\delta_{j} \otimes 1\right) \Pi\left(L^{\omega}(g)\right)\right)\left(f, g \in \mathfrak{A}_{I}, j \in I\right)$ is also dense in $H_{\rho}$.

Let $f_{i}, g_{i} \in \mathfrak{A}_{I}(i=1,2)$ and $j, j^{\prime} \in I$. Then

$$
\begin{aligned}
\left(\Lambda _ { \rho } \left(\Pi \left(L^{\omega}(\right.\right.\right. & \left.\left.f_{1}\right)\left(\delta_{j} \otimes 1\right) \Pi\left(L^{\omega}\left(f_{2}\right)\right)\right) \mid \Lambda_{\rho}\left(\Pi\left(L^{\omega}\left(g_{1}\right)\left(\delta_{j^{\prime}} \otimes 1\right) \Pi\left(L^{\omega}\left(g_{2}\right)\right)\right)\right) \\
& =\rho\left(\Pi\left(L^{\omega}\left(g_{2}\right)^{*}\right)\left(\delta_{j^{\prime}} \otimes 1\right) \Pi\left(L^{\omega}\left(g_{1}^{\sharp} * f_{1}\right)\right)\left(\delta_{j} \otimes 1\right) \Pi\left(L^{\omega}\left(f_{2}\right)\right)\right) \\
& =\theta\left(L^{\omega}\left(g_{2}\right)^{*} \Pi^{-1}\left(T_{B}\left(\left(\delta_{j^{\prime}} \otimes 1\right) \Pi\left(L^{\omega}\left(g_{1}^{\sharp} * f_{1}\right)\right)\left(\delta_{j} \otimes 1\right)\right)\right) L^{\omega}\left(f_{2}\right)\right) \\
& \left.=\theta\left(L^{\omega}\left(g_{2}\right)^{*} L^{\omega}\left(\chi_{\mathcal{T}_{j, j^{\prime}}}\left(g_{1}^{\sharp} * f_{1}\right)\right) L^{\omega}\left(f_{2}\right)\right) \quad \text { (by Lemma 6.2 }\right) \\
& =\left(L^{\omega}\left(\chi_{\mathcal{T}_{j, j^{\prime}}}\left(g_{1}^{\sharp} * f_{1}\right)\right) f_{2} \mid g_{2}\right) \\
& \left.=\left(\Pi\left(L^{\omega}\left(g_{1}^{\sharp} * f_{1}\right)\right)\left(\delta_{j} \otimes f_{2}\right) \mid \delta_{j^{\prime}} \otimes g_{2}\right) \quad \text { (by Lemma 6.3 }\right) \\
& =\left(\Pi\left(L^{\omega}\left(f_{1}\right)\right)\left(\delta_{j} \otimes f_{2}\right) \mid \Pi\left(L^{\omega}\left(g_{1}\right)\right)\left(\delta_{j^{\prime}} \otimes g_{2}\right)\right) .
\end{aligned}
$$

This implies that there exists a unitary $\mathcal{U}$ from $H_{\rho}$ onto $\ell^{2}(I) \otimes L^{2}(\mathcal{R})$ with the desired property.

Proposition 6.5. Let $\theta_{1}:=\theta \circ \widehat{E}_{B}$. There exists a unitary $\mathcal{U}_{1}$ from the Hilbert space $H_{\theta_{1}}$ obtained from the weight $\theta_{1}$ onto $\ell^{2}(I) \otimes L^{2}(\mathcal{R})$ such that

$$
\mathcal{U}_{1} L^{\omega}(f) \Lambda_{\theta_{1}}\left(\chi_{\mathcal{R}_{j}} L^{\omega}(g)\right)=\Pi\left(L^{\omega}(f)\right)\left(\delta_{j} \otimes g\right) \quad\left(f, g \in \mathfrak{A}_{I}, j \in I\right)
$$

and $\mathcal{U}_{1}$ implements the $*$-isomorphism $\Phi$.

Proof. Let $\rho:=\theta \circ \Pi^{-1} \circ T_{B}$ be as before. By the proof of [13, Lemma 2.4], the *-isomorphism $\Phi$ is implemented by the unitary $U: H_{\theta_{1}} \rightarrow H_{\rho}$ characterized by

$$
U \Lambda_{\theta_{1}}\left(a e_{B} b\right)=\Lambda_{\rho}\left(\Pi(a)\left(\delta_{0} \otimes 1\right) \Pi(b)\right) \quad(a, b \in A) .
$$

Let $\left\{v_{j, n}\right\}$ and $\left\{X_{n}\right\}$ be respectively the partial isometries in $\mathcal{G N}(D)$ and the Borel subsets of $X$ that were introduced in the proof of [3, Theorem 4.2]. So we have

$$
\Pi\left(v_{j, n}^{*}\right)\left(\delta_{0} \otimes 1\right) \Pi\left(v_{j, n}\right)=\delta_{j} \otimes \chi_{X_{n}}, \quad \sum_{n=1}^{\infty} \Pi\left(v_{j, n}^{*}\right)\left(\delta_{0} \otimes 1\right) \Pi\left(v_{j, n}\right)=\delta_{j} \otimes 1 .
$$

Thus the sequence $\left\{\sum_{n=1}^{N} \Pi\left(v_{j, n}^{*}\right)\left(\delta_{0} \otimes 1\right) \Pi\left(v_{j, n}\right)\right\}_{N=1}^{\infty}$ converges $\sigma$-strongly* to $\delta_{j} \otimes 1$. It follows that $\left\{\sum_{n=1}^{N} \Pi\left(L^{\omega}(f)\right) \Pi\left(v_{j, n}^{*}\right)\left(\delta_{0} \otimes 1\right) \Pi\left(v_{j, n}\right) \Pi\left(L^{\omega}(g)\right)\right\}_{N=1}^{\infty}$ converges $\sigma$-strongly* to $\Pi\left(L^{\omega}(f)\right)\left(\delta_{j} \otimes 1\right) \Pi\left(L^{\omega}(g)\right)$ for any $f, g \in \mathfrak{A}_{I}$. Applying the $*$-isomorphism $\Phi^{-1}$ discussed in Section 3 to the above sequence, we easily see that the sequence $\left\{\sum_{n=1}^{N} L^{\omega}(f) v_{j, n}^{*} e_{B} v_{j, n} L^{\omega}(g)\right\}_{N=1}^{\infty}$ converges $\sigma$-strongly* to $L^{\omega}(f) \chi_{\mathcal{R}_{j}} L^{\omega}(g)$. 
Fix any $f, g \in \mathfrak{A}_{I}$. By Lemma 6.4.

$$
\begin{aligned}
\sum_{n=1}^{N} \Lambda_{\rho}( & \left.\Pi\left(L^{\omega}(f)\right) \Pi\left(v_{j, n}^{*}\right)\left(\delta_{0} \otimes 1\right) \Pi\left(v_{j, n}\right) \Pi\left(L^{\omega}(g)\right)\right) \\
= & \sum_{n=1}^{N} \Lambda_{\rho}\left(\Pi\left(L^{\omega}(f)\right)\left(\delta_{j} \otimes \chi_{X_{n}}\right) \Pi\left(L^{\omega}(g)\right)\right) \\
= & \sum_{n=1}^{N} \Lambda_{\rho}\left(\Pi\left(L^{\omega}(f)\right)\left(\delta_{j} \otimes 1\right) \Pi\left(L^{\omega}\left(\chi_{X_{n}} g\right)\right)\right)=\sum_{n=1}^{N} \mathcal{U}^{*} \Pi\left(L^{\omega}(f)\right)\left(\delta_{j} \otimes \chi_{X_{n}} g\right) \\
& \rightarrow \mathcal{U}^{*} \Pi\left(L^{\omega}(f)\right)\left(\delta_{j} \otimes g\right)=\Lambda_{\rho}\left(\Pi\left(L^{\omega}(f)\right)\left(\delta_{j} \otimes 1\right) \Pi\left(L^{\omega}(g)\right)\right) \quad(N \rightarrow \infty) .
\end{aligned}
$$

Hence $\left\{\sum_{n=1}^{N} \Lambda_{\theta_{1}}\left(\Pi\left(L^{\omega}(f)\right) v_{j, n}^{*} e_{B} v_{j, n} L^{\omega}(g)\right)\right\}_{N=1}^{\infty}$ converges to

$$
U^{*} \mathcal{U}^{*} \Pi\left(L^{\omega}(f)\right)\left(\delta_{j} \otimes g\right)=U^{*} \Lambda_{\rho}\left(\Pi\left(L^{\omega}(f)\right)\left(\delta_{j} \otimes 1\right) \Pi\left(L^{\omega}(g)\right)\right) .
$$

Since $\Lambda_{\theta_{1}}$ is a $\sigma$-strongly* closed linear map (see [16, Introduction]), $L^{\omega}(f) \chi_{\mathcal{R}_{j}} L^{\omega}(g)$ belongs to $\mathfrak{n}_{\theta_{1}}$ and $\Lambda_{\theta_{1}}\left(L^{\omega}(f) \chi_{\mathcal{R}_{j}} L^{\omega}(g)\right)=U^{*} \Lambda_{\rho}\left(\Pi\left(L^{\omega}(f)\right)\left(\delta_{j} \otimes 1\right) \Pi\left(L^{\omega}(g)\right)\right)$. Therefore, if we set $\mathcal{U}_{1}:=\mathcal{U} U: H_{\theta_{1}} \rightarrow \ell^{2}(J) \otimes L^{2}(\mathcal{R})$, then

$$
\mathcal{U}_{1} L^{\omega}(f) \Lambda_{\theta_{1}}\left(\chi_{\mathcal{R}_{j}} L^{\omega}(g)\right)=\Pi\left(L^{\omega}(f)\right)\left(\delta_{j} \otimes g\right) \quad\left(f, g \in \mathfrak{A}_{I}, j \in I\right)
$$

and $\mathcal{U}_{1}$ implements $\Phi$.

Let $Z$ be in $A_{1} \cap B^{\prime}$. By [3, Lemma 4.4], $Z$ is a function in $L^{\infty}(\mathcal{R})$ satisfying $Z(x, u)=Z(y, u)$ and $Z(u, x)=Z(u, y)$ for a.e. $(x, y) \in \mathcal{S}$ and all $u \sim x$. By Theorem $3.2, \Phi(Z)$ is in $L^{\infty}(I \times X)^{\mathcal{S}}$.

Proposition 6.6. With the above notation, we have

$$
\Phi(Z)(j, x)=Z\left(x, \psi_{j}(x)\right) \quad(\text { a.e. }(j, x) \in I \times X) .
$$

Proof. By Proposition 6.5. we get, for any $f \in \mathfrak{A}_{I}$,

$$
\Phi(Z)\left(\delta_{j} \otimes f\right)=\mathcal{U}_{1} Z \mathcal{U}_{1}^{*}\left(\delta_{j} \otimes f\right)=\mathcal{U}_{1} \Lambda_{\theta_{1}}\left(Z \chi_{\mathcal{R}_{j}} L^{\omega}(f)\right)
$$

Now define a Borel function $h_{j}$ on $X$ by $h_{j}(x):=Z\left(x, \psi_{j}(x)\right)$. Then, since $(x, y) \in$ $\mathcal{R}_{j}$ if and only if $\left(\psi_{j}(x), y\right) \in \mathcal{S}$ for a.e. $(x, y) \in \mathcal{R}$, we have

$$
\begin{aligned}
\int_{\mathcal{R}}\left|Z(x, y)-h_{j}(x)\right| \chi_{\mathcal{R}_{j}} & (x, y) d \nu(x, y) \\
& =\int_{\mathcal{R}}\left|Z(x, y)-Z\left(x, \psi_{j}(x)\right)\right| \chi_{\mathcal{S}}\left(\psi_{j}(x), y\right) d \nu(x, y) \\
& =\int_{\mathcal{R}}|Z(x, y)-Z(x, y)| \chi_{\mathcal{S}}\left(\psi_{j}(x), y\right) d \nu(x, y)=0 .
\end{aligned}
$$


Thus $Z \chi_{\mathcal{R}_{j}}=h_{j} \chi_{\mathcal{R}_{j}}$. So we obtain

$$
\Phi(Z)\left(\delta_{j} \otimes f\right)=\mathcal{U}_{1} \Lambda_{\theta_{1}}\left(h_{j} \chi_{\mathcal{R}_{j}} L^{\omega}(f)\right)=\mathcal{U}_{1} \Lambda_{\theta_{1}}\left(\chi_{\mathcal{R}_{j}} L^{\omega}\left(h_{j} f\right)\right)=\delta_{j} \otimes h_{j} f .
$$

Since $f \in \mathfrak{A}_{I}$ and $j \in I$ are arbitrary, we get the desired identity.

We will return to our discussion of the properties of the map $\Phi: A_{1} \cap B^{\prime} \rightarrow$ $L^{\infty}(I \times X)^{\mathcal{S}}$ examined above in Section 9.2.

\section{$\S 7$. The dual operator valued weight of $T_{B}$}

By the result of Section 5, we know that the basic extension of the inclusion $\Pi(A) \subseteq W^{*}\left(I_{\sigma} \times \mathcal{R}, \omega^{\sigma}\right)$ is $B\left(\ell^{2}(I)\right) \otimes A$. The goal of this section is to study the dual operator valued weight $\widehat{T}_{B}$ of $T_{B}$.

As in the previous section, let $\rho:=\theta \circ \Pi^{-1} \circ T_{B}$, which is a faithful normal semifinite weight on $W^{*}\left(I_{\sigma} \times \mathcal{R}, \omega^{\sigma}\right)$.

Theorem 7.1. The dual operator valued weight $\widehat{T}_{B}$ of $T_{B}$ is the unique faithful normal conditional expectation from $B\left(\ell^{2}(I)\right) \otimes A$ onto $W^{*}\left(I_{\sigma} \times \mathcal{R}, \omega^{\sigma}\right)$. It satisfies $\rho \circ \widehat{T}_{B}=\operatorname{Tr} \otimes \theta$.

Proof. Put $\eta:=\operatorname{Tr} \otimes \theta$. For any $j \in I$, we have

$$
\left\{V_{\sigma}\left(\delta_{j} \otimes \xi_{0}\right)\right\}(i,(x, y))=\delta_{j}(\sigma(y, x)(i)) \xi_{0}(x, y)=\delta_{i, j} \xi_{0}(x, y)=\left(\delta_{j} \otimes \xi_{0}\right)(i,(x, y)) .
$$

Thus $V_{\sigma}\left(\delta_{j} \otimes \xi_{0}\right)=\delta_{j} \otimes \xi_{0}$. Since $\eta(S)=\sum_{j \in I}\left(S\left(\delta_{j} \otimes \xi_{0}\right) \mid \delta_{j} \otimes \xi_{0}\right)$ for any $S \in\left(B\left(\ell^{2}(I)\right) \otimes A\right)_{+}$, it follows that $\eta \circ \operatorname{Ad} V_{\sigma}^{*}=\eta$. Let $a \in A$. Then, by the result of Section 4 preceding Lemma 4.1 , we get

$$
\begin{aligned}
\eta\left(\Pi(a)^{*}\left(\delta_{0} \otimes 1\right) \Pi(a)\right) & =\eta\left(V_{\sigma}^{*} \Pi(a)^{*}\left(\delta_{0} \otimes 1\right) \Pi(a) V_{\sigma}\right) \\
& =\theta\left((\operatorname{Tr} \otimes \mathrm{id})\left(V_{\sigma}^{*} \Pi(a)^{*}\left(\delta_{0} \otimes 1\right) \Pi(a) V_{\sigma}\right)\right) \\
& =\theta\left(a^{*} a\right)=\rho\left(\Pi(a)^{*}\left(\delta_{0} \otimes 1\right) \Pi(a)\right)<\infty .
\end{aligned}
$$

This implies that the restriction of $\eta$ to $W^{*}\left(I_{\sigma} \times \mathcal{R}, \omega^{\sigma}\right)$ is semifinite. It is clear that $\sigma_{t}^{\eta}=\operatorname{Ad}\left(1 \otimes \delta^{i t}\right)$. It follows that the restriction of $\sigma_{t}^{\eta}$ to $W^{*}\left(I_{\sigma} \times \mathcal{R}, \omega^{\sigma}\right)$ equals $\sigma_{t}^{\rho}$. In particular, $\sigma_{t}^{\eta}\left(W^{*}\left(I_{\sigma} \times \mathcal{R}, \omega^{\sigma}\right)\right)=W^{*}\left(I_{\sigma} \times \mathcal{R}, \omega^{\sigma}\right)$ for any $t \in \mathbb{R}$. By [20], there exists a unique faithful normal conditional expectation $F$ from $B\left(\ell^{2}(I)\right) \otimes A$ onto $W^{*}\left(I_{\sigma} \times \mathcal{R}, \omega^{\sigma}\right)$ such that $\eta \circ F=\eta$.

Let $\rho_{0}:=\left.\eta\right|_{W^{*}\left(I_{\sigma} \times \mathcal{R}, \omega^{\sigma}\right)}$. By the result of the previous paragraph, $\rho_{0}$ equals $\rho$ on the $\sigma$-strongly* dense subalgebra $\operatorname{span}\left\{\Pi(a)^{*}\left(\delta_{0} \otimes 1\right) \Pi(b): a, b \in A\right\}$ which is contained in $\mathfrak{m}_{\rho}$. Moreover, we have

$$
\sigma_{t}^{\rho_{0}}=\left.\sigma_{t}^{\eta}\right|_{W^{*}\left(I_{\sigma} \times \mathcal{R}, \omega^{\sigma}\right)}=\left.\operatorname{Ad}\left(1 \otimes \delta^{i t}\right)\right|_{W^{*}\left(I_{\sigma} \times \mathcal{R}, \omega^{\sigma}\right)}=\sigma_{t}^{\rho} .
$$

We thus find that $\rho=\rho_{0}$. Hence $\rho \circ F=\eta$. 
We prove below that $F$ is exactly the dual operator valued weight $\widehat{T}_{B}$ of $T_{B}$. (Because $\left(B\left(\ell^{2}(I)\right) \otimes A\right) \cap W^{*}\left(I_{\sigma} \times \mathcal{R}, \omega^{\sigma}\right)^{\prime}=\mathbb{C}$, it follows from 5, Proposition 11.1] that $\widehat{T}_{B}$ is proportional to $F$. Hence $\widehat{T}_{B}$ is in particular bounded.) Let $\zeta:=\theta \circ \Pi^{-1}$, which is a faithful normal state on $\Pi(A)$. By the result of [5, p. 514], we have

$$
\frac{d \rho \circ \widehat{T}_{B}}{d \zeta^{\prime}}=\frac{d \zeta \circ T_{B} \circ \widehat{T}_{B}}{d \zeta^{\prime}}=\Delta_{\rho}=1 \otimes \delta=\frac{d(\operatorname{Tr} \otimes \theta)}{d\left(t_{\mathbb{C}} \otimes \theta^{\prime}\right)},
$$

where $\zeta^{\prime}$ is a weight (state) on $\tilde{J} \Pi(A) \tilde{J}$ given by $\zeta^{\prime}(S):=\zeta(\tilde{J} S \tilde{J})\left(S \in(\tilde{J} \Pi(A) \tilde{J})_{+}\right)$, $t_{\mathbb{C}}$ is a state on the one-dimensional von Neumann algebra of the scalar multiples of the identity on $\ell^{2}(I)$ defined by $t_{\mathbb{C}}\left(c \cdot 1_{\ell^{2}(I)}\right)=c$, and $\theta^{\prime}$ is a weight on $A^{\prime}$ defined by $\theta^{\prime}(b):=\theta(J b J)\left(b \in\left(A^{\prime}\right)_{+}\right)$. We find from the result of Section 5 that $\tilde{J} \Pi(A) \tilde{J}=\mathbb{C} \otimes A^{\prime}$ and $\zeta^{\prime}=t_{\mathbb{C}} \otimes \theta^{\prime}$. So we have

$$
\frac{d \rho \circ \widehat{T}_{B}}{d\left(t_{\mathbb{C}} \otimes \theta^{\prime}\right)}=\frac{d \rho \circ \widehat{T}_{B}}{d \zeta^{\prime}}=\frac{d(\operatorname{Tr} \otimes \theta)}{d\left(t_{\mathbb{C}} \otimes \theta^{\prime}\right)} .
$$

Hence $\rho \circ \widehat{T}_{B}=\operatorname{Tr} \otimes \theta$. From the previous paragraph, it follows that $\rho \circ F=\rho \circ \widehat{T}_{B}$. By [18, we conclude that $F=\widehat{T}_{B}$.

Remark. Let $\widehat{\mathcal{R}}:=I^{2} \times \mathcal{R}$, which we consider as the product of the (transitive and principal) equivalence relation $I^{2}$ and $\mathcal{R}$. As is well known, the groupoid von Neumann algebra $W^{*}\left(\widehat{\mathcal{R}}, \mathrm{id}_{I^{2}} \times \omega\right)$ is isomorphic to $B\left(\ell^{2}(I)\right) \otimes A$. The skewproduct relation $I_{\sigma} \times \mathcal{R}$ is in this case a Borel subrelation of $\widehat{\mathcal{R}}$. Hence there is a faithful normal conditional expectation from $W^{*}\left(\widehat{\mathcal{R}}, \mathrm{id}_{I^{2}} \times \omega\right)=B\left(\ell^{2}(I)\right) \otimes A$ onto $W^{*}\left(I_{\sigma} \times \mathcal{R}, \omega^{\sigma}\right)$ (see [1]). Because $\left(B\left(\ell^{2}(I)\right) \otimes A\right) \cap W^{*}\left(I_{\sigma} \times \mathcal{R}, \omega^{\sigma}\right)^{\prime}=\mathbb{C}$, this expectation is exactly the dual operator valued weight $\widehat{T}_{B}$.

Lemma 7.2. The restriction of $\widehat{T}_{B}$ to $\left(B\left(\ell^{2}(I)\right) \otimes A\right) \cap \Pi(A)^{\prime}$ equals that of the faithful normal expectation $E_{\tilde{D}}$ from $B\left(\ell^{2}(J)\right) \otimes A$ onto $\tilde{D}:=\ell^{\infty}(I) \otimes D$ (which is the Cartan subalgebra for both $B\left(\ell^{2}(I)\right) \otimes A$ and $\left.W^{*}\left(I_{\sigma} \times \mathcal{R}, \omega^{\sigma}\right)\right)$.

Proof. Since $\tilde{D}$ is maximal abelian in $B\left(\ell^{2}(I)\right) \otimes A$, there is only one faithful normal expectation from $B\left(\ell^{2}(I)\right) \otimes A$ onto $\tilde{D}$. It follows that we have $E^{\sigma} \circ \widehat{T}_{B}=E_{\tilde{D}}$. Let $S \in\left(B\left(\ell^{2}(I)\right) \otimes A\right) \cap \Pi(A)^{\prime}$. Then, since $\Pi(A)$ is a factor, $\widehat{T}_{B}(S)$ is of the form $\widehat{T}_{B}(S)=c \cdot 1$ for some $c \in \mathbb{C}$. So we get

$$
E_{\tilde{D}}(S)=E^{\sigma}\left(\widehat{T}_{B}(S)\right)=c \cdot 1=\widehat{T}_{B}(S) .
$$

This completes the proof. 
Theorem 7.3. The restriction of $\widehat{T}_{B}$ to the relative commutant $\left(B\left(\ell^{2}(I)\right) \otimes A\right) \cap$ $\Pi(A)^{\prime}$ is given by

$$
R \in\left(B\left(\ell^{2}(I)\right) \otimes A\right) \cap \Pi(A)^{\prime} \mapsto\left(\int_{X} R_{j, j}(x) d m(x)\right) \cdot 1
$$

for any probability measure $m$ on $X$ equivalent to $\mu$, where $\left\{R_{i, j}(x)\right\}_{i, j \in I, x \in X}$ the decomposition of $R \in\left(B\left(\ell^{2}(I)\right) \otimes A\right) \cap \Pi(A)^{\prime}$ as stated in Theorem 5.1 .

Proof. Let $R=\left\{R_{i, j}(x)\right\}$ be as in the assertion, so it satisfies 5.1. By Lemma 7.2. $\widehat{T}_{B}(R)=E_{\tilde{D}}(R)$ is the function given by

$$
x \in X \mapsto\left[\delta_{i, j} R_{j, j}(x)\right]_{i, j \in I} \in \ell^{\infty}(I) \subseteq B\left(\ell^{2}(I)\right) .
$$

Now we define a bounded measurable function $f$ on $I \times X$ by $f(j, x):=R_{j, j}(x)$. By (5.1), we have $f(\sigma(y, x)(j), y)=f(j, x)$ for a.e. $(j, x)$. This means that $f$ is an $\left(I_{\sigma} \times \mathcal{R}\right)$-invariant function. Since $I_{\sigma} \times \mathcal{R}$ is ergodic, $f$ is constant. Hence we obtain

$$
\widehat{T}_{B}(R)=\left(\int_{X} R_{j, j}(x) d m(x)\right) \cdot 1
$$

for any $j \in I$ and any probability measure $m$ on $X$ equivalent to $\mu$.

We conclude this section with a result about normality.

For each $j \in I$, define

$$
\mathcal{C}_{j}:=\left\{(x, y) \in \mathcal{R}: \exists z \in X,(x, z) \in \mathcal{S} \text { and }\left(\psi_{j}(z), y\right) \in \mathcal{S}\right\} .
$$

Remark that $\mathcal{C}_{j}$ is measurable. Indeed, let $H$ be a countable group in the full group $[\mathcal{S}]:=\left\{\rho \in[\mathcal{S}]_{*}: \operatorname{Dom}(\rho)=\operatorname{Im}(\rho)=X\right\}$ such that $\mathcal{S}=\{(x, h(x)): x \in X, h \in H\}$ (cf. [6, Theorem 1]). Then it is easily checked that

$$
\mathcal{C}_{j}=\bigcup_{h_{1}, h_{2} \in H} \Gamma\left(h_{1} \circ \psi_{j} \circ h_{2}\right)
$$

Thus $\mathcal{C}_{j}$ is measurable.

Note that the definition of $\left\{\mathcal{C}_{j}\right\}$ obviously depends on the choice of the choice functions $\left\{\psi_{j}\right\}$. Clearly, $\mathcal{R}=\bigcup_{j \in I} \mathcal{C}_{j}$ up to a null set. It is easily verified that $\mathcal{R}_{j} \subseteq \mathcal{C}_{j}$ for all $j \in I$.

Lemma 7.4. Each $\chi_{\mathcal{C}_{j}}$ belongs to $A_{1} \cap B^{\prime}$.

Proof. Let $(x, y) \in \mathcal{S}$ and $z \in X$ be such that $z \sim x$.

Suppose that $(x, z) \in \mathcal{C}_{j}$. Choose $w \in X$ satisfying $(x, w) \in \mathcal{S}$ and $\left(\psi_{j}(w), z\right)$ $\in \mathcal{S}$. Then $(y, w)=(y, x)(x, w) \in \mathcal{S}$. Thus $(y, z) \in \mathcal{C}_{j}$. 
Conversely, suppose that $(y, z) \in \mathcal{C}_{j}$. Choose $u \in X$ satisfying $(y, u) \in \mathcal{S}$ and $\left(\psi_{j}(u), z\right) \in \mathcal{S}$. Then $(x, u)=(x, y)(y, u) \in \mathcal{S}$. So $(x, z) \in \mathcal{C}_{j}$.

By the results of the previous two paragraphs, we obtain $\chi_{\mathcal{C}_{j}}(x, z)=\chi_{\mathcal{C}_{j}}(y, z)$ whenever $(x, y) \in \mathcal{S}$ and $z \sim x$. Similarly, one can check that $\chi_{\mathcal{C}_{j}}(z, x)=\chi_{\mathcal{C}_{j}}(z, y)$ whenever $(x, y) \in \mathcal{S}$ and $z \sim x$. By [3, Lemma 4.4], $\chi_{\mathcal{C}_{j}}$ belongs to $A_{1} \cap B^{\prime}$.

Lemma 7.5. Let $j \in I$. The following are equivalent:

(1) $\chi_{\mathcal{R}_{j}}=\chi_{\mathcal{C}_{j}}$, that is, $\nu\left(\mathcal{C}_{j} \backslash \mathcal{R}_{j}\right)=0$;

$(2)$ the Borel map $\psi_{j}$ normalizes $\mathcal{S}:\left(\psi_{j}(x), \psi_{j}(y)\right) \in \mathcal{S}$ for a.e. $(x, y) \in \mathcal{S}$.

Proof. $(1) \Rightarrow(2)$ : By assumption, there exists a $\mu$-conull Borel subset $X_{0}$ of $X$ such that $r^{-1}(x) \cap\left(\mathcal{C}_{j} \backslash \mathcal{R}_{j}\right)=\emptyset$ for all $x \in X_{0}$. Put $\mathcal{S}_{0}:=\mathcal{S} \cap\left(X_{0} \times X_{0}\right)$. Then $\nu\left(\mathcal{S} \backslash \mathcal{S}_{0}\right)=0$. Let $(x, y) \in \mathcal{S}_{0}$. Then $\left(x, \psi_{j}(y)\right) \in \mathcal{C}_{j}$, because $(x, y) \in \mathcal{S}$ and $\left(\psi_{j}(y), \psi_{j}(y)\right) \in \mathcal{S}$. Since $x \in X_{0}$ and $\left(x, \psi_{j}(y)\right) \in r^{-1}(x) \cap \mathcal{C}_{j}$, it follows that $\left(x, \psi_{j}(y)\right) \in \mathcal{R}_{j}$. Thus, $\left(\psi_{j}(x), \psi_{j}(y)\right) \in \mathcal{S}$.

$(2) \Rightarrow(1)$ : Choose a $\mu$-conull Borel subset $X_{1}$ of $X$ such that $\left(\psi_{j}(x), \psi_{j}(y)\right) \in \mathcal{S}$ for all $(x, y) \in \mathcal{S}$ with $x \in X_{1}$. Put $\mathcal{C}_{j}^{\prime}:=\mathcal{C}_{j} \cap\left(X_{1} \times X\right)$. Then $\nu\left(\mathcal{C}_{j} \backslash \mathcal{C}_{j}^{\prime}\right)=0$. Let $(x, y) \in \mathcal{C}_{j}^{\prime}$. So $(x, z) \in \mathcal{S}$ and $\left(\psi_{j}(z), y\right) \in \mathcal{S}$ for some $z \in X$. Because $\left(\psi_{j}(x), \psi_{j}(z)\right) \in \mathcal{S}$, one gets $\left(\psi_{j}(x), y\right) \in \mathcal{S}$. This means that $(x, y) \in \mathcal{R}_{j}$. Hence $\mathcal{C}_{j}^{\prime} \subseteq \mathcal{R}_{j}$. Therefore, $\nu\left(\mathcal{C}_{j} \backslash \mathcal{R}_{j}\right)=0$.

Lemma 7.6. If $j_{1}, j_{2} \in I$ satisfy $\nu\left(\Gamma\left(\psi_{j_{1}}\right) \cap \mathcal{C}_{j_{2}}\right)=0$, then $\nu\left(\mathcal{C}_{j_{1}} \cap \mathcal{C}_{j_{2}}\right)=0$.

Proof. By assumption, there exists a $\mu$-conull Borel subset $X_{0}$ of $X$ such that $\left\{\left(x, \psi_{j_{1}}(x)\right): x \in X_{0}\right\} \subseteq\left(\mathcal{C}_{j_{2}}\right)^{c}$. Let $\mathcal{C}_{1}:=\left\{(x, y) \in \mathcal{C}_{j_{1}}: \exists z \in X_{0},(x, z) \in \mathcal{S}\right.$ and $\left.\left(\psi_{j_{1}}(z), y\right) \in \mathcal{S}\right\}$. Then $\mathcal{C}_{j_{1}} \backslash \mathcal{C}_{1}=\left\{(x, y) \in \mathcal{C}_{j_{1}}: \exists z \in X_{0}^{c},(x, z) \in \mathcal{S}\right.$ and $\left.\left(\psi_{j_{1}}(z), y\right) \in \mathcal{S}\right\}$. If $\left\{h_{n}\right\}$ is a countable family of Borel isomorphisms in $[\mathcal{S}]$ with $\mathcal{S}=\bigcup_{n} \Gamma\left(h_{n}\right)$, then we see that $r\left(\mathcal{C}_{j_{1}} \backslash \mathcal{C}_{1}\right)=\bigcup_{n} h_{n}\left(X_{0}^{c}\right)$. Hence $r\left(\mathcal{C}_{j_{1}} \backslash \mathcal{C}_{1}\right)$ is $\nu$-null. In particular, $\nu\left(\mathcal{C}_{j_{1}} \backslash \mathcal{C}_{1}\right)=0$. Hence $\nu\left(\mathcal{C}_{j_{1}} \cap \mathcal{C}_{j_{2}}\right)=\nu\left(\mathcal{C}_{1} \cap \mathcal{C}_{j_{2}}\right)$.

Suppose that $\mathcal{C}_{1} \cap \mathcal{C}_{j_{2}}$ is non-empty, and take $(x, y) \in \mathcal{C}_{1} \cap \mathcal{C}_{j_{2}}$. So $(x, z) \in \mathcal{S}$ and $\left(\psi_{j_{1}}(z), y\right) \in \mathcal{S}$ for some $z \in X_{0}$. Since $(x, z) \in \mathcal{S}$ and $\psi_{j_{1}}(z) \sim x$, we have

$$
\chi_{\mathcal{C}_{j_{2}}}\left(x, \psi_{j_{1}}(z)\right)=\chi_{\mathcal{C}_{j_{2}}}\left(z, \psi_{j_{1}}(z)\right)=0 \quad\left(\left(z, \psi_{j_{1}}(z)\right) \notin \mathcal{C}_{j_{2}}\right) .
$$

This shows that $\left(x, \psi_{j_{1}}(z)\right) \notin \mathcal{C}_{j_{2}}$. Since $\left(\psi_{j_{1}}(z), y\right) \in \mathcal{S}$ and $x \sim y$, we get

$$
\chi_{\mathcal{C}_{j_{2}}}(x, y)=\chi_{\mathcal{C}_{j_{2}}}\left(x, \psi_{j_{1}}(z)\right)=0 .
$$

So $(x, y) \notin \mathcal{C}_{j_{2}}$, a contradiction. Therefore, $\mathcal{C}_{1} \cap \mathcal{C}_{j_{2}}=\emptyset$.

Define $P_{j}:=\Phi\left(\chi_{\mathcal{C}_{j}}\right)$. So each $P_{j}$ is a projection in $L^{\infty}(I \times X)^{\mathcal{S}}$. From Proposition 6.6 and the fact that $\Gamma\left(\psi_{j}\right) \subseteq \mathcal{C}_{j}$, we have $P_{j}(j, x)=\chi_{\mathcal{C}_{j}}\left(x, \psi_{j}(x)\right)=1$. 
Lemma 7.7. Let $j \in I$. The following are equivalent:

(1) $T_{B}\left(P_{j}\right)=1$;

(2) the map $\psi_{j}$ normalizes $\mathcal{S}$.

Proof. By Corollary 4.3 , we have

$$
\begin{aligned}
T_{B}\left(P_{j}\right) & =\left(\int_{X} \sum_{i \in I} \chi_{\mathcal{C}_{j}}\left(x, \psi_{i}(x)\right) d \mu(x)\right) \cdot 1 \\
& =\left(\int_{X}\left(\chi_{\mathcal{C}_{j}}\left(x, \psi_{j}(x)\right)+\sum_{i \in I \backslash\{j\}} \chi_{\mathcal{C}_{j}}\left(x, \psi_{i}(x)\right)\right) d \mu(x)\right) \cdot 1 \\
& =\left(\int_{X}\left(1+\sum_{i \in I \backslash\{j\}} \chi_{\mathcal{C}_{j}}\left(x, \psi_{i}(x)\right)\right) d \mu(x)\right) \cdot 1 \\
& =1+\left(\int_{X}\left(\sum_{i \in I \backslash\{j\}} \chi_{\mathcal{C}_{j}}\left(x, \psi_{i}(x)\right)\right) d \mu(x)\right) \cdot 1
\end{aligned}
$$

Hence we find that

$$
\begin{aligned}
T_{B}\left(P_{j}\right)=1 & \Leftrightarrow \chi_{\mathcal{C}_{j}}\left(x, \psi_{i}(x)\right)=0(\forall i \in I \backslash\{j\} \text { and a.e. } x \in X) \\
& \Leftrightarrow \nu\left(\Gamma\left(\psi_{i}\right) \cap \mathcal{C}_{j}\right)=0(\forall i \in I \backslash\{j\}) \\
& \Leftrightarrow \nu\left(\mathcal{C}_{i} \cap \mathcal{C}_{j}\right)=0(\forall i \in I \backslash\{j\}) \quad \text { (by Lemma 7.6). }
\end{aligned}
$$

$(1) \Rightarrow(2)$ : Suppose that $T_{B}\left(P_{j}\right)=1$. By the result of the previous paragraph, we have $\nu\left(\mathcal{C}_{i} \cap \mathcal{C}_{j}\right)=0$ for all $i \in I \backslash\{j\}$. In particular, $\nu\left(\mathcal{R}_{i} \cap \mathcal{C}_{j}\right)=0$, i.e., $\chi_{\mathcal{R}_{i}} \chi_{\mathcal{C}_{j}}=0$. Since $\sum_{i \in I} \chi_{\mathcal{R}_{i}}=1$, it follows that

$$
\chi_{\mathcal{C}_{j}}=\sum_{i \in I} \chi_{\mathcal{C}_{j}} \chi_{\mathcal{R}_{i}}=\chi_{\mathcal{C}_{j}} \chi_{\mathcal{R}_{j}}=\chi_{\mathcal{R}_{j}} .
$$

We now see from Lemma 7.5 that $\psi_{j}$ normalizes $\mathcal{S}$.

$(2) \Rightarrow(1)$ : As in the proof of Lemma $7.5(2) \Rightarrow(1)$, there is a $\mu$-conull Borel subset $X_{1}$ of $X$ such that $\left(\psi_{j}(x), \psi_{j}(y)\right) \in \mathcal{S}$ for all $(x, y) \in \mathcal{S}$ with $x \in X_{1}$. Suppose that $\nu\left(\mathcal{C}_{i} \cap \mathcal{R}_{j}\right)>0$ for some $i \in I \backslash\{j\}$. Then the set $\mathcal{C}_{i} \cap \mathcal{R}_{j} \cap\left(X_{1} \times X\right)$ still has positive measure. Take any $(x, y)$ in this set. Then there is a $z \in X$ such that $(x, z) \in \mathcal{S}$ and $\left(\psi_{i}(z), y\right) \in \mathcal{S}$. We also have $\left(\psi_{j}(x), y\right) \in \mathcal{S}$. Thus $\left(\psi_{j}(x), \psi_{i}(z)\right) \in \mathcal{S}$. Meanwhile, since $x \in X_{1}$ and $(x, z) \in \mathcal{S}$, we have $\left(\psi_{j}(x), \psi_{j}(z)\right) \in \mathcal{S}$. Consequently, $\left(\psi_{i}(z), \psi_{j}(z)\right) \in \mathcal{S}$, which contradicts the fact that $\left\{\psi_{i}\right\}$ are choice functions. Therefore, we must have $\nu\left(\mathcal{C}_{i} \cap \mathcal{R}_{j}\right)=0$ for all $i \in I \backslash\{j\}$. Since $\Gamma\left(\psi_{j}\right) \subseteq \mathcal{R}_{j}$, one has $\nu\left(\Gamma\left(\psi_{j}\right) \cap \mathcal{C}_{i}\right)=0$. By Lemma 7.6. we get $\nu\left(\mathcal{C}_{j} \cap \mathcal{C}_{i}\right)=0$. From the result of the first paragraph, it follows that $T_{B}\left(P_{j}\right)=1$. 


\section{$\S 8$. Fourier transform}

Throughout this section, we assume that the commensurability subrelation $\operatorname{Comm}_{\mathcal{R}}(\mathcal{S})$ of $\mathcal{S}$ in $\mathcal{R}$ equals $\mathcal{R}$. Equivalently, we suppose that the irreducible inclusion $B \subseteq A$ is discrete. Hence the restriction of the operator valued weight $T_{B}$ to $L^{\infty}(I \times X)^{\mathcal{S}}$ is semifinite. We denote by $\Omega$ the faithful normal semifinite trace on $M:=L^{\infty}(I \times X)^{\mathcal{S}}$ induced by the restriction of $T_{B}$ to $M$. By Corollary 4.3 , we have

$$
\Omega(F)=\int_{X} \sum_{j \in I} F(j, x) d \mu(x) \quad\left(\forall F \in\left(L^{\infty}(I \times X)^{\mathcal{S}}\right)_{+}\right) .
$$

In the discussion below, we employ the notation used in Section 5 .

Let $L:=\left(B\left(\ell^{2}(I)\right) \otimes A\right) \cap \Pi(B)^{\prime}$. By arguing as in Section 5, we can easily verify that $L$ consists of all essentially bounded Borel functions $S: X \rightarrow B\left(\ell^{2}(I)\right)$ such that, with the matrix representation $S(x)=\left[S_{i, j}(x)\right]_{i, j \in I}(x \in X)$, one has

$$
S_{i, \sigma(x, y)(j)}(x)=S_{\sigma(y, x)(i), j}(y) \quad(\text { a.e. }(x, y) \in \mathcal{S}) .
$$

Let $S=\left[S_{i, j}(x)\right] \in L$ and $F \in \mathfrak{n}_{\Omega}$. Then define a Borel function $S * F$ on $I \times X$ by

$$
(S * F)(j, x):=\sum_{k \in I} S_{j, k}(x) F(k, x) .
$$

Since $\{F(k, x)\}_{k \in I}$ belongs to $\ell^{2}(I)$ for a.e. $x \in X$ due to the fact that $F \in \mathfrak{n}_{\Omega}$, it follows that $\{(S * F)(j, x)\}_{j \in I}$ is a member of $\ell^{2}(I)$ for a.e. $x \in X$. In particular, we have $S * F \in L^{\infty}(I \times X)$. Moreover, for a.e. $(x, y) \in \mathcal{S}$,

$$
\begin{aligned}
(S * F)(\sigma(y, x)(j), y) & =\sum_{k \in I} S_{\sigma(y, x)(j), k}(y) F(k, y)=\sum_{k \in I} S_{j, \sigma(x, y)(k)}(x) F(k, y) \\
& =\sum_{k \in I} S_{j, k}(x) F(\sigma(y, x)(k), y)=\sum_{k \in I} S_{j, k}(x) F(k, x) \\
& =(S * F)(j, x) .
\end{aligned}
$$

Thus $S * F$ satisfies $(3.2)$. Hence it belongs to $M$. In fact, it is in $\mathfrak{n}_{\Omega}$, because

$$
\begin{aligned}
\Omega\left((S * F)^{*}(S * F)\right)^{1 / 2} & \\
& \left.=\text { the } \ell^{2}(I) \text {-norm of the sequence }\{(S * F)(j, x)\}_{j \in I} \text { (for a.e. } x \in X\right) \\
& =\text { the } \ell^{2}(I) \text {-norm of the vector } S(x)\{F(j, x)\}_{j \in I} \in \ell^{2}(I) \text { (for a.e. } x \in X \text { ) } \\
& \leq\|S\| \|\left\{(F(j, x)\}_{j \in I}\left\|_{\ell^{2}(I)}=\right\| S\|\| \Lambda_{\Omega}(F) \|<\infty .\right.
\end{aligned}
$$

This in particular shows that $\left\|\Lambda_{\Omega}(S * F)\right\| \leq\|S\|\left\|\Lambda_{\Omega}(F)\right\|$. Therefore, the map

$$
\Lambda_{\Omega}(F) \in H_{\Omega} \mapsto \Lambda_{\Omega}(S * F) \in H_{\Omega}
$$

extends uniquely to a bounded linear operator $\hat{\lambda}(S)$ on $H_{\Omega}$. 
Proposition 8.1. The map $\hat{\lambda}: L \rightarrow B\left(H_{\Omega}\right)$ is a surjective unital normal *representation of $L$.

Proof. It is easy to verify that $\hat{\lambda}$ is a unital $*$-representation that is continuous with respect to the weak (hence $\sigma$-weak) topology. In particular, $\hat{\lambda}(L)$ is a von Neumann subalgebra of $B\left(H_{\Omega}\right)$. Let $F_{1}, F_{2} \in \mathfrak{n}_{\Omega}$. For each $x \in X$ and $(i, j) \in I \times I$, define

$$
S_{i, j}^{0}(x):=F_{1}(i, x) \overline{F_{2}(j, x)} .
$$

Since $\sum_{i, j \in I}\left|S_{i, j}^{0}(x)\right|^{2}=\left(\sum_{i \in I}\left|F_{1}(i, x)\right|^{2}\right)\left(\sum_{j \in I}\left|F_{2}(j, x)\right|^{2}\right)<\infty$ for a.e. $x \in X$, the map

$$
g=\{g(j)\}_{j \in I} \in \ell^{2}(I) \mapsto\left\{\sum_{j \in I} S_{i, j}^{0}(x) g(j)\right\}_{i \in I} \in \ell^{2}(I)
$$

defines a bounded operator $S^{0}(x) \in B\left(\ell^{2}(I)\right)$ for a.e. $x \in X$ with $\left\|S^{0}(x)\right\| \leq$ $\left\|\Lambda_{\Omega}\left(F_{1}\right)\right\|\left\|\Lambda_{\Omega}\left(F_{2}\right)\right\|$. Hence the essentially bounded Borel function $S^{0}: x \in X \mapsto$ $S^{0}(x) \in B\left(\ell^{2}(I)\right)$ belongs to $B\left(\ell^{2}(I)\right) \otimes D$. It is easy to see that $S^{0}$ satisfies 8.1). So it is in $L$. It is also easy to check that $\hat{\lambda}\left(S^{0}\right)=t_{\Lambda_{\Omega}\left(F_{1}\right), \Lambda_{\Omega}\left(F_{2}\right)}$, where, in general, the symbol $t_{\xi, \eta}$ stands for the rank-one operator on a Hilbert space $K$ defined by $t_{\xi, \eta} \zeta:=(\zeta \mid \eta) \xi(\xi, \eta, \zeta \in K)$. Since $\hat{\lambda}(L)$ is a von Neumann algebra, it follows from the above argument that $\hat{\lambda}(L)$ contains all the rank-one (hence finite-rank) operators on $H_{\Omega}$. Therefore, $\hat{\lambda}(L)$ coincides with $B\left(H_{\Omega}\right)$.

We denote by $\widehat{\Omega}$ the faithful normal state on $\widehat{M}:=\left(B\left(\ell^{2}(I)\right) \otimes A\right) \cap \Pi(A)^{\prime}$ induced by restricting $\widehat{T}_{B}$ to $\left(B\left(\ell^{2}(I)\right) \otimes A\right) \cap \Pi(A)^{\prime}$.

We define, for each $R=\left[R_{i, j}(x)\right] \in \widehat{M}$, a function $F^{R}$ on $I \times X$ by

$$
\left.F^{R}(k, x):=R_{k, 0}(x) \quad((k, x) \in I \times X)\right) .
$$

Since

$$
F^{R}(k, x)=\left(R(x) \delta_{k} \mid \delta_{0}\right),
$$

$F^{R}$ is Borel and bounded, so that it belongs to the Cartan subalgebra $\ell^{\infty}(I) \otimes D$ of $W^{*}\left(I_{\sigma} \times \mathcal{R}, \omega^{\sigma}\right)$.

Lemma 8.2. The operator $F^{R}$ constructed above belongs to $M$. In particular, it is in $\mathfrak{n}_{\Omega}$.

Proof. To show that $F^{R}$ is in $M$, it suffices to check that it satisfies 3.2 . But this immediately follows from (5.1). Moreover, we have 


$$
\begin{aligned}
\Omega\left(\left(F^{R}\right)^{*} F^{R}\right) & =\Omega\left(\left|F^{R}\right|^{2}\right)=\int_{X} \sum_{k \in I}\left|F^{R}(k, x)\right|^{2} d \mu(x)=\int_{X} \sum_{k \in I}\left|R_{k, 0}(x)\right|^{2} d \mu(x) \\
& =\int_{X} \sum_{k \in I} \overline{R_{k, 0}(x)} R_{k, 0} d \mu(x)=\int_{X} \sum_{k \in I}\left(R^{*}\right)_{0, k}(x) R_{k, 0}(x) d \mu(x) \\
& =\int_{X}\left(R^{*} R\right)_{0,0}(x) d \mu(x)=\widehat{\Omega}\left(R^{*} R\right)<\infty .
\end{aligned}
$$

Thus $F^{R}$ belongs to $\mathfrak{n}_{\Omega}$.

Remark. The domain of the map : $R \in \widehat{M} \mapsto F^{R} \in M$ can be extended to $L$ by the same formula: $F^{S}(j, x)=S_{j, 0}(x)(S \in L)$. The computation in the proof of Lemma 8.2 shows that $F^{S}$ defined this way from $S \in L$ still belongs to $\mathfrak{n}_{\Omega}$, since $x \in X \mapsto\left(S^{*} S\right)_{0,0}(x)$ is a constant function due to its $\mathcal{S}$-invariance.

By the computation in the proof of Lemma 8.2 , the map

$$
\Lambda_{\widehat{\Omega}}(R) \mapsto \Lambda_{\Omega}\left(F^{R}\right) \quad(R \in \widehat{M})
$$

extends to an isometry $\mathcal{F}$ from $H_{\widehat{\Omega}}$ into $H_{\Omega}$. We call $\mathcal{F}$ the Fourier transform associated with the pair $\mathcal{S} \subseteq \mathcal{R}$.

We claim that the map $\mathcal{F}$ is in fact unitary. To prove our claim, we will first show the following:

Lemma 8.3. Let $F \in \mathfrak{n}_{\Omega}$ and $R_{i, j}^{F}(x):=F\left(\sigma\left(\psi_{j}(x), x\right)(i), \psi_{j}(x)\right)(i, j \in I, x \in X)$. Suppose that

(1) for any $g \in \ell^{2}(I),\left\{\sum_{j \in I} R_{i, j}^{F}(x) g(j)\right\}_{i \in I}$ belongs to $\ell^{2}(I)$ for a.e. $x \in X$;

(2) the linear map $g \in \ell^{2}(I) \mapsto\left\{\sum_{j \in I} R_{i, j}^{F}(x) g(j)\right\}_{i \in I} \in \ell^{2}(I)$ is bounded for a.e. $x \in X$.

Then $R^{F}=\left[R_{i, j}^{F}(x)\right]_{i, j \in I}$ belongs to $\widehat{M}$ and $\mathcal{F} \Lambda_{\hat{\Omega}}\left(R^{F}\right)=\Lambda_{\Omega}(F)$.

Proof. By assumption, $\left[R_{i, j}^{F}(x)\right]_{i, j \in I}$ defines an element in $B\left(\ell^{2}(I)\right)$ for a.e. $x \in X$. Since $F$ is Borel, the assignment $x \in X \mapsto\left[R_{i, j}^{F}(x)\right]_{i, j \in I} \in B\left(\ell^{2}(I)\right)$ is an essentially bounded Borel map, so defines an element $R^{F}=\left[R_{i, j}^{F}(x)\right]_{i, j \in I}$ in $B\left(\ell^{2}(I)\right) \otimes D$. In order to prove that $R^{F}$ is in $\widehat{M}$, it suffices to show that $R^{F}$ satisfies (5.1). But this can be verified by a direct computation which is left to the reader. Finally, $R_{j, 0}^{F}(x)=F(\sigma(x, x)(j), x)=F(j, x)$. Therefore, $\mathcal{F} \Lambda_{\hat{\Omega}}\left(R^{F}\right)=\Lambda_{\Omega}(F)$.

We shall define a partition of unity in $M$ which satisfies the assumptions of Lemma 8.3 .

Thanks to [2, Theorem 3.8], we may choose our choice functions $\left\{\psi_{i}\right\}_{i \in I}$ so that they enjoy the following properties: 
(1) There exist a countable set $\Lambda$ and natural numbers $\left\{n_{\lambda}\right\}_{\lambda \in \Lambda}$ such that the index set $I$ is equal to $\left\{(\lambda, n): \lambda \in \Lambda, n=1, \ldots, n_{\lambda}\right\}$.

(2) $\operatorname{Ind}\left(\psi_{\lambda, n}\right)=n_{\lambda}$ for each $(\lambda, n) \in I$.

(3) For each $\lambda \in \Lambda$ and $n, m \in\left\{1, \ldots, n_{\lambda}\right\}, \mathcal{S}\left(\psi_{\lambda, n}(\mathcal{S}(x))\right)=\mathcal{S}\left(\psi_{\lambda, m}(\mathcal{S}(x))\right)$ for a.e. $x \in X$. Moreover, $\mathcal{S}\left(\psi_{\lambda, n}(\mathcal{S}(x))\right)$ is the disjoint union of $\left\{\mathcal{S}\left(\psi_{\lambda, k}(x)\right)\right\}_{k=1}^{n_{\lambda}}$.

We refer to 2 for the definition and the basic properties of the index $\operatorname{Ind}(\rho)$ of a "nonsingular" map $\rho$. In what follows, we fix choice functions $\left\{\psi_{\lambda, n}\right\}$ as above.

Let $\mathcal{C}_{j}(j \in I)$ be the $\mathcal{S}$-invariant set introduced in Section 7. A direct computation shows that, for a.e. $(x, y) \in \mathcal{R}$ and $(\lambda, n) \in I,(x, y)$ is in $\mathcal{C}_{\lambda, n}$ if and only if $y \in \bigcup_{m=1}^{n_{\lambda}} \mathcal{S}\left(\psi_{\lambda, m}(x)\right)$. It is easy to check that $\mathcal{C}_{\lambda, n}$ is equal to $\mathcal{C}_{\lambda, m}$ up to a null set. Put $\mathcal{C}_{\lambda}:=\mathcal{C}_{\lambda, 1}$. We note that $\left\{\mathcal{C}_{\lambda}\right\}_{\lambda \in \Lambda}$ is a measurable partition of $\mathcal{R}$, because $\left\{\chi_{\mathcal{C}_{\lambda}}\right\}_{\lambda \in \Lambda}$ are the minimal projections in $M$ satisfying $\sum_{\lambda \in \Lambda} \chi_{\mathcal{C}_{\lambda}}=1$ (see the proof of [2, Theorem 3.8]).

Put $F^{\lambda}:=\Phi\left(\chi_{\mathcal{C}_{\lambda}}\right)$ for each $\lambda \in \Lambda$, where $\Phi$ is defined in Proposition 6.5. By Proposition 6.6, $F^{\lambda}$ is defined by

$$
F^{\lambda}\left(\left(\lambda^{\prime}, n\right), x\right)=\chi_{\mathcal{C}_{\lambda}}\left(x, \psi_{\lambda^{\prime}, n}(x)\right) \quad\left(\left(\left(\lambda^{\prime}, n\right), x\right) \in I \times X\right) .
$$

In particular, $F^{\lambda}$ belongs to $M$.

Lemma 8.4. $\left\{F^{\lambda}\right\}_{\lambda \in \Lambda}$ is the set of minimal projections in $M$ with $\sum_{\lambda \in \Lambda} F^{\lambda}=1$, and $\Omega\left(F^{\lambda}\right)=n_{\lambda}$ for each $\lambda \in \Lambda$.

Proof. Since $\left\{\chi_{\mathcal{C}_{\lambda}}\right\}_{\lambda \in \Lambda}$ is the set of minimal projections in $M=A_{1} \cap B^{\prime}$ with sum equal to the identity (see [2, Theorem 3.8]), $\left\{F^{\lambda}\right\}_{\lambda \in \Lambda}$ are in turn the minimal projections in $M$ with $\sum_{\lambda \in \Lambda} F^{\lambda}=1$. Moreover, a direct computation shows that

$$
\begin{aligned}
\Omega\left(F^{\lambda}\right) & =\int_{X} \sum_{\lambda^{\prime} \in \Lambda} \sum_{n=1}^{n_{\lambda^{\prime}}} \chi_{\mathcal{C}_{\lambda}}\left(x, \psi_{\lambda^{\prime}, n}(x)\right) d \mu(x) \\
& =\int_{X} \sum_{n=1}^{n_{\lambda}} \chi_{\mathcal{C}_{\lambda}}\left(x, \psi_{\lambda, n}(x)\right) d \mu(x)=n_{\lambda} .
\end{aligned}
$$

Let $\lambda \in \Lambda$ and $i \in I$. Define a function $f_{i}^{\lambda}$ on $X$ by

$$
f_{i}^{\lambda}(x):=\sum_{j \in I} \chi_{\mathcal{C}_{\lambda}}\left(\psi_{i}(x), \psi_{j}(x)\right)
$$

Lemma 8.5. There exists a $\mu$-null subset $N$ of $X$ such that $f_{i}^{\lambda}(x)=n_{\lambda}$ for all $x \in N^{c}, \lambda \in \Lambda$ and all $i \in I$. 
Proof. By (the proof of) [2, Theorem 3.8] (see part (3) stated above), there exists a $\mu$-null subset $N_{0}$ of $X$ such that $\mathcal{R}(x)=\bigsqcup_{i \in I} \mathcal{S}\left(\psi_{i}(x)\right)$ and

(8.2) $\mathcal{S}\left(\psi_{\lambda_{1}, n_{1}}(\mathcal{S}(x))\right)=\bigsqcup_{k=1}^{n_{\lambda_{1}}} \mathcal{S}\left(\psi_{\lambda_{1}, k}(x)\right) \quad$ for all $x \in N_{0}^{c}$ and all $\left(\lambda_{1}, n_{1}\right) \in I$.

Here $\bigsqcup$ stands for disjoint union of sets. Put $N:=\bigcup_{i \in I} \psi_{i}^{-1}\left(N_{0}\right)$. Let $x \in N^{c}$. Then we have

$$
\begin{aligned}
\left(\psi_{i}(x), \psi_{j}(x)\right) & \in \mathcal{C}_{\lambda} \\
& \Leftrightarrow \psi_{j}(x) \in \mathcal{S}\left(\psi_{\lambda, 1}\left(\mathcal{S}\left(\psi_{i}(x)\right)\right)\right) \\
& \left.\left.\Leftrightarrow \exists k \in\left\{1, \ldots, n_{\lambda}\right\},\left(\psi_{j}(x), \psi_{\lambda, k}\left(\psi_{i}(x)\right)\right) \in \mathcal{S} \quad \text { (by } 8.2\right)\right) \\
& \Leftrightarrow \exists k \in\left\{1, \ldots, n_{\lambda}\right\}, j=\sigma\left(x, \psi_{i}(x)\right)(\lambda, k) .
\end{aligned}
$$

Thus $f_{i}^{\lambda}(x)=n_{\lambda}$ for all $x \in N^{c}, \lambda \in \Lambda$ and $i \in I$.

Lemma 8.6. Each $F^{\lambda}$ satisfies the two conditions in Lemma 8.3.

Proof. Let $f_{i}^{\lambda}(x)$ be as before, and $N$ be the $\mu$-null set of $X$ as in Lemma 8.5 Since each $\chi_{\mathcal{C}_{\lambda}}$ is minimal, for each $\lambda \in \Lambda$ there exists $\lambda^{-1} \in \Lambda$ which satisfies $\mathcal{C}_{\lambda^{-1}}=\mathcal{C}_{\lambda}^{-1}$. Now, as in Lemma 8.3. consider $\left\{R_{i, j}^{F^{\lambda}}\right\}_{i, j \in I}$. Then, for each $g \in \ell^{2}(I)$ and any $x \in N^{c}$, we have

$$
\begin{aligned}
\left\|R^{F^{\lambda}}(x) g\right\|^{2} & =\sum_{i \in I}\left|\sum_{j \in I} R_{i, j}^{F^{\lambda}}(x) g(j)\right|^{2}=\sum_{i \in I}\left|\sum_{j \in I} \chi_{\mathcal{C}_{\lambda}}\left(\psi_{j}(x), \psi_{i}(x)\right) g(j)\right|^{2} \\
& =\sum_{i \in I}\left|\sum_{j \in I} \chi_{\mathcal{C}_{\lambda^{-1}}}\left(\psi_{i}(x), \psi_{j}(x)\right) g(j)\right|^{2} \\
& =\sum_{i \in I}\left|\sum_{k=1}^{n_{\lambda^{-1}}} g\left(\sigma\left(x, \psi_{i}(x)\right)\left(\lambda^{-1}, k\right)\right)\right|^{2} \\
& \leq \sum_{i \in I} n_{\lambda^{-1}} \sum_{k=1}^{n_{\lambda^{-1}}}\left|g\left(\sigma\left(x, \psi_{i}(x)\right)\left(\lambda^{-1}, k\right)\right)\right|^{2} \\
& =n_{\lambda^{-1}} \sum_{j \in I} \sum_{i \in I}\left|\chi_{\mathcal{C}_{\lambda}}\left(\psi_{j}(x), \psi_{i}(x)\right) g(j)\right|^{2} \\
& =n_{\lambda^{-1}} \sum_{j \in I}|g(j)|^{2} \sum_{i \in I} \chi_{\mathcal{C}_{\lambda}}\left(\psi_{j}(x), \psi_{i}(x)\right) \\
& =n_{\lambda^{-1}} \sum_{j \in I}|g(j)|^{2} f_{j}^{\lambda}(x)=n_{\lambda} n_{\lambda^{-1}}\|g\|^{2} .
\end{aligned}
$$

Therefore we get the conclusion. 
By using the above lemmas, we get the following:

Theorem 8.7. The Fourier transform $\mathcal{F}$ is unitary.

Proof. From Lemma 8.4 and the fact that $\sum_{\lambda \in \Lambda} F^{\lambda}=1$, we find that $\operatorname{span}\left\{F^{\lambda}\right.$ : $\lambda \in \Lambda\}$, the linear span of $\left\{F^{\lambda}: \lambda \in \Lambda\right\}$, is a $\sigma$-strongly* dense $*$-subalgebra of $M$ contained in $\mathfrak{n}_{\Omega}$. In particular, $\operatorname{span}\left\{\Lambda_{\Omega}\left(F^{\lambda}\right): \lambda \in \Lambda\right\}$ is dense in $H_{\Omega}$. Meanwhile, we know from Lemmas 8.3 and 8.6 that $\mathcal{F} \Lambda_{\hat{\Omega}}\left(R^{F^{\lambda}}\right)=\Lambda_{\Omega}\left(F^{\lambda}\right)$ for all $\lambda \in \Lambda$. It thus follows that $\mathcal{F}$ has dense range. Since $\mathcal{F}$ is an isometry, it must be unitary.

Theorem 8.8. The restriction of the normal representation $\hat{\lambda}$ to $\widehat{M}$, still denoted by $\hat{\lambda}$, is implemented by the Fourier transform $\mathcal{F}$, i.e., $\hat{\lambda}(R)=\mathcal{F} R \mathcal{F}^{*}$ for all $R \in \widehat{M}$.

Proof. Let $R \in \widehat{M}$ be arbitrary and $\left\{F^{\lambda}\right\}_{\lambda \in \Lambda}$ be as before. Then

$$
\mathcal{F} R \mathcal{F}^{*} \Lambda_{\Omega}\left(F^{\lambda}\right)=\mathcal{F} R \Lambda_{\hat{\Omega}}\left(R^{F^{\lambda}}\right)=\mathcal{F} \Lambda_{\hat{\Omega}}\left(R R^{F^{\lambda}}\right)=\Lambda_{\Omega}\left(F^{R R^{F^{\lambda}}}\right) .
$$

By definition, we have

$$
\begin{aligned}
F^{R R^{F^{\lambda}}}(i, x) & =\left(R R^{F^{\lambda}}\right)_{i, 0}(x)=\sum_{j \in I} R_{i, j}(x) R_{j, 0}^{F^{\lambda}}(x) \\
& =\sum_{j \in I} R_{i, j}(x) F^{\lambda}(j, x)=\left(R * F^{\lambda}\right)(i, x) .
\end{aligned}
$$

Thus $F^{R R^{F^{\lambda}}}=R * F^{\lambda}$. Hence we get

$$
\mathcal{F} R \mathcal{F}^{*} \Lambda_{\Omega}\left(F^{\lambda}\right)=\Lambda_{\Omega}\left(R * F^{\lambda}\right)=\hat{\lambda}(R) \Lambda_{\Omega}\left(F^{\lambda}\right) .
$$

Since the linear span of $\left\{\Lambda_{\Omega}\left(F^{\lambda}\right): \lambda \in \Lambda\right\}$ is dense in $H_{\Omega}$ as noted before, we conclude that $\hat{\lambda}(R)=\mathcal{F} R \mathcal{F}^{*}$.

Thanks to Theorem 8.8 , we will often identify $\widehat{M}$ with the image $\hat{\lambda}(\widehat{M})$ and regard $\widehat{M}$ as acting on the Hilbert space $H_{\Omega}$.

\section{§9. Hecke algebras in the setting of measured equivalence relations}

As before, let $\mathcal{S}$ be a Borel subrelation $\mathcal{S}$ of $\mathcal{R}$.

\section{§9.1. A characterization of the index for "nonsingular" maps}

In this subsection, we develop the theory of index which is defined in [2].

For each measurable nonsingular map $\rho$ which satisfies $\mu(\operatorname{Dom}(\rho))>0$ and $\Gamma(\rho) \subseteq \mathcal{R}$, we define

$$
\rho^{-1} \mathcal{S} \rho:=\left\{(x, y) \in \mathcal{R}_{\operatorname{Dom}(\rho)}:(\rho(x), \rho(y)) \in \mathcal{S}\right\} .
$$


It is easy to check that $\rho^{-1} \mathcal{S} \rho$ is a measured equivalence subrelation of $\mathcal{R}_{\operatorname{Dom}(\rho)}$ and for a.e. $x \in \operatorname{Dom}(\rho)$,

$$
\rho\left(\rho^{-1} \mathcal{S} \rho(x)\right)=\mathcal{S}(\rho(x)) \cap \operatorname{Im}(\rho) .
$$

Put $\mathcal{S}_{\rho}:=\mathcal{S} \cap \rho^{-1} \mathcal{S} \rho$. We shall show that the index of $\rho$ is determined by the inclusion $\mathcal{S}_{\rho} \subseteq \mathcal{S}_{\operatorname{Dom}(\rho)}$.

Proposition 9.1. In the above setting, if $\mathcal{S}$ is ergodic, then $\operatorname{Ind}(\rho)=\left[\mathcal{S}_{\operatorname{Dom}(\rho)}: \mathcal{S}_{\rho}\right]$.

Proof. Suppose that $\left[\mathcal{S}_{\operatorname{Dom}(\rho)}: \mathcal{S}_{\rho}\right]=n$. By [2, Lemma 3.3], it suffices to show that there exist a nonnull subset $X_{1}$ of $\operatorname{Dom}(\rho)$ and countable measurable nonsingular maps $\left\{\rho_{k}\right\}_{k=1}^{n}$ on $X_{1}$ such that $\mathcal{S}(\rho(\mathcal{S}(x)))$ is equal to the disjoint union of $\mathcal{S}\left(\rho_{k}(x)\right)$ for all $x \in X_{1}$. By definition, there exist choice functions $\left\{\psi_{k}\right\}_{k=1}^{n}$ for $\mathcal{S}_{\rho} \subseteq \mathcal{S}_{\operatorname{Dom}(\rho)}$, i.e., there exists a $\mu$-conull Borel subset $X_{1}$ of $\operatorname{Dom}(\rho)$ such that $\mathcal{S}_{\operatorname{Dom}(\rho)}(x)=$ $\bigsqcup_{k=1}^{n} \mathcal{S}_{\rho}\left(\psi_{k}(x)\right)$ for all $x \in X_{1}$. A direct computation shows that for each $x \in X_{1}$,

$$
\begin{aligned}
\rho\left(\mathcal{S}_{\operatorname{Dom}(\rho)}(x)\right) & =\rho\left(\bigcup_{k=1}^{n}\left(\rho^{-1} \mathcal{S} \rho\left(\psi_{k}(x)\right)\right) \cap \mathcal{S}\left(\psi_{k}(x)\right)\right) \\
& =\bigcup_{k=1}^{n}\left(\mathcal{S}\left(\rho\left(\psi_{k}(x)\right)\right) \cap \operatorname{Im}(\rho) \cap \rho\left(\mathcal{S}\left(\psi_{k}(x)\right)\right)\right) \\
& \subseteq \bigcup_{k=1}^{n} \mathcal{S}\left(\rho\left(\psi_{k}(x)\right)\right) .
\end{aligned}
$$

On the other hand, since the graph of each $\psi_{k}$ is contained in $\mathcal{S}$, it follows that $\mathcal{S}\left(\rho\left(\psi_{k}(x)\right)\right) \subseteq \mathcal{S}(\rho(\mathcal{S}(x)))$ for each $x \in X_{1}$ and $k=1, \ldots, n$. Hence $\mathcal{S}(\rho(\mathcal{S}(x)))$ is the disjoint union of $\left\{\mathcal{S}\left(\rho\left(\psi_{k}(x)\right)\right)\right\}_{k=1}^{n}$ for all $x \in X_{1}$, and $\left\{\rho_{k}:=\left.\rho \circ \psi_{k}\right|_{X_{1}}\right\}_{k=1}^{n}$ has the desired properties.

Corollary 9.2. Suppose that $\mathcal{S} \subseteq \mathcal{R}$ is an inclusion of ergodic discrete measured equivalence relations. Then the commensurability groupoid $\operatorname{Comm}_{\mathcal{R}}(\mathcal{S})$ is generated by the countable union of $\left\{\Gamma\left(\rho_{n}\right)\right\}_{n=1}^{\infty}$ up to a null set, where each element $\rho_{n}$ belongs to $[\mathcal{R}]_{*}$ and

$$
\left[\mathcal{S}_{\mathrm{Dom}\left(\rho_{n}\right)}: \mathcal{S}_{\rho_{n}}\right]<\infty, \quad\left[\mathcal{S}_{\operatorname{Im}\left(\rho_{n}\right)}: \mathcal{S}_{\rho_{n}^{-1}}\right]<\infty .
$$

Proof. This is a direct consequence of [2, Theorem 3.7] and Proposition 9.1.

Hence our definition of commensurability is a natural generalization of the definition in group theory.

We note that the index satisfies the following: 
Lemma 9.3. Suppose that $\mathcal{P} \subseteq \mathcal{S}$ is an inclusion of ergodic equivalence subrelations of $\mathcal{R}$ and $\rho$ is in $[\mathcal{R}]_{*}$. Then

$$
\left[\rho^{-1} \mathcal{S} \rho: \rho^{-1} \mathcal{P} \rho\right]=\left[\mathcal{S}_{\operatorname{Im}(\rho)}: \mathcal{P}_{\operatorname{Im}(\rho)}\right]
$$

Proof. Suppose that $\left\{\psi_{i}\right\}_{i \in I}$ are choice functions for $\mathcal{P}_{\operatorname{Im}(\rho)} \subseteq \mathcal{S}_{\operatorname{Im}(\rho)}$. We will show that $\left\{\rho^{-1} \psi_{i} \rho\right\}_{i \in I}$ are choice functions for $\rho^{-1} \mathcal{P} \rho \subseteq \rho^{-1} \mathcal{S} \rho$. Indeed, there exists a $\mu$-null subset $X_{0}$ of $\operatorname{Im}(\rho)$ such that $\mathcal{S}_{\operatorname{Im}(\rho)}(x)$ is equal to the disjoint union of $\left\{\mathcal{P}_{\operatorname{Im}(\rho)}\left(\psi_{i}(x)\right)\right\}_{i \in I}$ for each $x \in \operatorname{Im}(\rho) \backslash X_{0}$. Put $X_{1}:=\rho^{-1}\left(X_{0}\right)$. Then $X_{1}$ is a $\mu$-null subset of $\operatorname{Dom}(\rho)$. Moreover, for each $x \in \operatorname{Dom}(\rho) \backslash X_{1}$,

$$
\mathcal{S}_{\operatorname{Im}(\rho)}(\rho(x))=\bigsqcup_{i \in I} \mathcal{P}_{\operatorname{Im}(\rho)}\left(\psi_{i}(\rho(x))\right) .
$$

By using 9.1, it follows that

$$
\begin{aligned}
\rho^{-1} \mathcal{S} \rho(x) & =\rho^{-1}\left(\mathcal{S}_{\operatorname{Im}(\rho)}(\rho(x)) \cap \operatorname{Im}(\rho)\right)=\rho^{-1}\left(\bigsqcup_{i \in I} \mathcal{P}_{\operatorname{Im}(\rho)}\left(\psi_{i}(\rho(x))\right) \cap \operatorname{Im}(\rho)\right) \\
& =\bigsqcup_{i \in I} \rho^{-1} \mathcal{P} \rho\left(\rho^{-1} \psi_{i} \rho(x)\right) .
\end{aligned}
$$

Hence our claim is proven.

Suppose that $\mathcal{S}$ is commensurable in $\mathcal{R}$ with a partition $\left\{\mathcal{C}_{\lambda}\right\}_{\lambda \in \Lambda}$ of $\mathcal{R}$ as before. We shall show that there exists a canonical 1-cocycle $c$ on $\mathcal{R}$ to the set of positive rational numbers.

Fix $\lambda \in \Lambda$. For $\lambda_{1}, \lambda_{2} \in \Lambda$, we define a Borel subset $\mathcal{C}_{\lambda, \lambda_{1}, \lambda_{2}}$ of $\mathcal{C}_{\lambda}$ by

$$
\mathcal{C}_{\lambda, \lambda_{1}, \lambda_{2}}:=\mathcal{C}_{\lambda} \cap \bigcup\left\{\Gamma\left(\rho_{2} \rho_{1}\right): \Gamma\left(\rho_{1}\right) \subseteq \mathcal{C}_{\lambda_{1}}, \Gamma\left(\rho_{2}\right) \subseteq \mathcal{C}_{\lambda_{2}}\right\}
$$

Put $\mathcal{C}_{\lambda}^{\prime}:=\mathcal{C}_{\lambda} \backslash \bigcup\left\{\mathcal{C}_{\lambda, \lambda_{1}, \lambda_{2}}: \lambda_{1}, \lambda_{2} \in \Lambda, \nu\left(\mathcal{C}_{\lambda, \lambda_{1}, \lambda_{2}}\right)=0\right\}$. It is easy to check that $\mathcal{C}_{\lambda}^{\prime}$ is conull in $\mathcal{C}_{\lambda}$, and $\bigcup_{\lambda \in \Lambda} \mathcal{C}_{\lambda}^{\prime}$ is conull in $\mathcal{R}$.

Now, we define a function $c$ on $\mathcal{R}$ by

$$
c(x, y):= \begin{cases}n_{\lambda^{-1}} / n_{\lambda}, & (x, y) \in \mathcal{C}_{\lambda}^{\prime}, \\ 1, & \text { otherwise }\end{cases}
$$

It is easy to check that $c$ is measurable. Moreover, we get the following:

Proposition 9.4. The function $c$ is a 1-cocycle on $\mathcal{R}$. Moreover, $c$ does not depend on the choice of $\left\{\mathcal{C}_{\lambda}\right\}_{\lambda \in \Lambda}$ of $\mathcal{R}$ up to null sets.

Proof. A direct computation shows that $\mathcal{C}_{\lambda, \lambda_{1}, \lambda_{2}}^{-1}=\mathcal{C}_{\lambda^{-1}, \lambda_{2}^{-1}, \lambda_{1}^{-1}}$ for all $\lambda, \lambda_{1}, \lambda_{2} \in \Lambda$, so $c(x, y)=c(y, x)^{-1}$ for each $(x, y) \in \mathcal{R}$. 
We claim that $c(x, y) c(y, z)=c(x, z)$ if $(x, y) \in \mathcal{C}_{\lambda_{1}}^{\prime},(y, z) \in \mathcal{C}_{\lambda_{2}}^{\prime}$ and $(x, z) \in$ $\mathcal{C}_{\lambda_{3}}^{\prime}$. Indeed, since $(x, z) \in \mathcal{C}_{\lambda_{3}, \lambda_{1}, \lambda_{2}} \cap \mathcal{C}_{\lambda_{3}}$, we get $\nu\left(\mathcal{C}_{\lambda_{3}, \lambda_{1}, \lambda_{2}}\right)>0$. So, by the definition of $\mathcal{C}_{\lambda}$ together with the arguments given in [2], there exist $\rho_{1}, \rho_{2} \in[\mathcal{R}]_{*}$ which satisfy the following:

(1) $x \in \operatorname{Dom}\left(\rho_{1}\right), \rho_{1}(x)=y$,

(2) $y \in \operatorname{Dom}\left(\rho_{2}\right), \rho_{2}(y)=z$,

(3) $\Gamma\left(\rho_{1}\right) \subseteq \mathcal{C}_{\lambda_{1}}^{\prime}, \Gamma\left(\rho_{2}\right) \subseteq \mathcal{C}_{\lambda_{2}}^{\prime} . \Gamma\left(\rho_{2} \rho_{1}\right) \subseteq \mathcal{C}_{\lambda_{3}}^{\prime}$.

It follows that $\operatorname{Ind}\left(\rho_{2} \rho_{1}\right)=n_{\lambda_{3}}, \operatorname{Ind}\left(\rho_{1}^{-1} \rho_{2}^{-1}\right)=n_{\lambda_{3}^{-1}}, \operatorname{Ind}\left(\rho_{i}\right)=n_{\lambda_{i}}$ and $\operatorname{Ind}\left(\rho_{i}^{-1}\right)$ $=n_{\lambda_{i}^{-1}}$ for $i=1,2$. By Proposition 9.1 and Lemma 9.3 , we have

$$
\begin{aligned}
& {\left[\mathcal{S}_{\rho_{2} \rho_{1}}: \mathcal{S}_{\rho_{1}} \cap \mathcal{S}_{\rho_{2} \rho_{1}}\right]=\left[\mathcal{S} \cap \rho_{1}^{-1} \rho_{2}^{-1} \mathcal{S} \rho_{2} \rho_{1}: \mathcal{S} \cap \rho_{1}^{-1} \mathcal{S} \rho_{1} \cap \rho_{1}^{-1} \rho_{2}^{-1} \mathcal{S} \rho_{2} \rho_{1}\right]} \\
& =\left[\rho_{2} \rho_{1} \mathcal{S} \rho_{1}^{-1} \rho_{2}^{-1} \cap \mathcal{S}: \rho_{2} \rho_{1} \mathcal{S} \rho_{1}^{-1} \rho_{2}^{-1} \cap \rho_{2} \mathcal{S} \rho_{2}^{-1} \cap \mathcal{S}\right] \\
& =\left[\mathcal{S}_{\rho_{1}^{-1} \rho_{2}^{-1}}: \mathcal{S}_{\rho_{1}^{-1} \rho_{2}^{-1}} \cap \mathcal{S}_{\rho_{2}^{-1}}\right], \\
& {\left[\mathcal{S}_{\operatorname{Dom}\left(\rho_{2} \rho_{1}\right)}: \mathcal{S}_{\rho_{1}} \cap \mathcal{S}_{\rho_{2} \rho_{1}}\right]=\left[\mathcal{S}_{\operatorname{Dom}\left(\rho_{2} \rho_{1}\right)}: \mathcal{S}_{\rho_{1}}\right]\left[\mathcal{S}_{\rho_{1}}: \mathcal{S}_{\rho_{1}} \cap \mathcal{S}_{\rho_{2} \rho_{1}}\right]} \\
& =\left[\mathcal{S}_{\mathrm{Dom}\left(\rho_{1}\right)}: \mathcal{S}_{\rho_{1}}\right]\left[\mathcal{S}_{\rho_{1}}: \mathcal{S}_{\rho_{1}} \cap \mathcal{S}_{\rho_{2} \rho_{1}}\right] \\
& =n_{\lambda_{1}}\left[\mathcal{S} \cap \rho_{1}^{-1} \mathcal{S} \rho_{1}: \mathcal{S} \cap \rho_{1}^{-1} \mathcal{S} \rho_{1} \cap \rho_{1}^{-1} \rho_{2}^{-1} \mathcal{S} \rho_{2} \rho_{1}\right] \\
& =n_{\lambda_{1}}\left[\rho_{1} \mathcal{S} \rho_{1}^{-1} \cap \mathcal{S}: \rho_{1} \mathcal{S} \rho_{1}^{-1} \cap \mathcal{S} \cap \rho_{2}^{-1} \mathcal{S} \rho_{2}\right] \\
& =n_{\lambda_{1}}\left[\mathcal{S}_{\rho_{1}^{-1}}: \mathcal{S}_{\rho_{1}^{-1}} \cap \mathcal{S}_{\rho_{2}}\right]=\frac{n_{\lambda_{1}}}{n_{\lambda_{1}^{-1}}}\left[\mathcal{S}_{\operatorname{Dom}\left(\rho_{1}^{-1}\right)}: \mathcal{S}_{\rho_{1}^{-1}} \cap \mathcal{S}_{\rho_{2}}\right] \\
& =\frac{n_{\lambda_{1}}}{n_{\lambda_{1}^{-1}}}\left[\mathcal{S}_{\operatorname{Dom}\left(\rho_{2}\right)}: \mathcal{S}_{\rho_{1}^{-1}} \cap \mathcal{S}_{\rho_{2}}\right] \\
& =\frac{n_{\lambda_{1}} n_{\lambda_{2}}}{n_{\lambda_{1}^{-1}}}\left[\mathcal{S}_{\rho_{2}}: \mathcal{S}_{\rho_{1}^{-1}} \cap \mathcal{S}_{\rho_{2}}\right] \\
& =\frac{n_{\lambda_{1}} n_{\lambda_{2}}}{n_{\lambda_{1}^{-1}}}\left[\mathcal{S} \cap \rho_{2}^{-1} \mathcal{S} \rho_{2}: \mathcal{S} \cap \rho_{1} \mathcal{S} \rho_{1}^{-1} \cap \rho_{2}^{-1} \mathcal{S} \rho_{2}\right] \\
& =\frac{n_{\lambda_{1}} n_{\lambda_{2}}}{n_{\lambda_{1}^{-1}}}\left[\rho_{2} \mathcal{S} \rho_{2}^{-1} \cap \mathcal{S}: \rho_{2} \mathcal{S} \rho_{2}^{-1} \cap \rho_{2} \rho_{1} \mathcal{S} \rho_{1}^{-1} \rho_{2}^{-1} \cap \mathcal{S}\right] \\
& =\frac{n_{\lambda_{1}} n_{\lambda_{2}}}{n_{\lambda_{1}^{-1}}}\left[\mathcal{S}_{\rho_{2}^{-1}}: \mathcal{S}_{\rho_{1}^{-1} \rho_{2}^{-1}} \cap \mathcal{S}_{\rho_{2}^{-1}}\right]
\end{aligned}
$$

Hence we conclude that

$$
\begin{aligned}
c(x, z)=\frac{n_{\lambda_{3}^{-1}}}{n_{\lambda_{3}}} & =\frac{\left[\mathcal{S}_{\operatorname{Dom}\left(\rho_{1}^{-1} \rho_{2}^{-1}\right)}: \mathcal{S}_{\rho_{1}^{-1} \rho_{2}^{-1}}\right]}{\left[\mathcal{S}_{\operatorname{Dom}\left(\rho_{2} \rho_{1}\right)}: \mathcal{S}_{\rho_{2} \rho_{1}}\right]} \\
& =\frac{\left[\mathcal{S}_{\operatorname{Dom}\left(\rho_{2}^{-1}\right)}: \mathcal{S}_{\rho_{1}^{-1} \rho_{2}^{-1}}\right]\left[\mathcal{S}_{\rho_{2} \rho_{1}}: \mathcal{S}_{\rho_{1}} \cap \mathcal{S}_{\rho_{2} \rho_{1}}\right]}{\left[\mathcal{S}_{\operatorname{Dom}\left(\rho_{2} \rho_{1}\right)}: \mathcal{S}_{\rho_{1}} \cap \mathcal{S}_{\rho_{2} \rho_{1}}\right]}
\end{aligned}
$$




$$
\begin{aligned}
& =\frac{\left.n_{\lambda_{1}^{-1}}\left[\mathcal{S}_{\operatorname{Dom}\left(\rho_{2}^{-1}\right)}: \mathcal{S}_{\left.\rho_{1}^{-1} \rho_{2}^{-1}\right]}\right] \mathcal{S}_{\rho_{1}^{-1} \rho_{2}^{-1}}: \mathcal{S}_{\rho_{1}^{-1} \rho_{2}^{-1}} \cap \mathcal{S}_{\rho_{2}^{-1}}\right]}{n_{\lambda_{1}} n_{\lambda_{2}}\left[\mathcal{S}_{\rho_{2}^{-1}}: \mathcal{S}_{\rho_{1}^{-1} \rho_{2}^{-1}} \cap \mathcal{S}_{\rho_{2}^{-1}}\right]} \\
& =\frac{n_{\lambda_{1}^{-1}}\left[\mathcal{S}_{\operatorname{Dom}\left(\rho_{2}^{-1}\right)}: \mathcal{S}_{\rho_{2}^{-1}}\right]}{n_{\lambda_{1}} n_{\lambda_{2}}}=\frac{n_{\lambda_{1}^{-1}} n_{\lambda_{2}^{-1}}}{n_{\lambda_{1}} n_{\lambda_{2}}}=c(x, y) c(y, z) .
\end{aligned}
$$

So our claim is proven.

The "moreover" assertion follows from the fact that $\left\{\mathcal{C}_{\lambda}\right\}_{\lambda \in \Lambda}$ is canonically determined. Indeed, for another partition $\left\{\mathcal{D}_{\delta}\right\}_{\delta \in \Delta}$, by using the proof of [2, Theorem 3.8], there exists a bijective map $\Pi$ from $\Delta$ to $\Lambda$ with a $\nu$-null set $\mathcal{R}_{1}$ in $\mathcal{R}$ which satisfies $\Pi\left(\delta^{-1}\right)=\Pi(\delta)^{-1}$ and $\mathcal{D}_{\delta} \backslash \mathcal{R}_{1}=\mathcal{C}_{\Pi(\delta)} \backslash \mathcal{R}_{1}$ for each $\delta \in \Delta$. In particular, we have $n_{\Pi(\delta)}=n_{\delta}$ and $\mathcal{D}_{\delta, \delta_{1}, \delta_{2}} \backslash \mathcal{R}_{1}=\mathcal{C}_{\Pi(\delta), \Pi\left(\delta_{1}\right), \Pi\left(\delta_{2}\right)} \backslash \mathcal{R}_{1}$ for each $\delta, \delta_{1}, \delta_{2} \in \Delta$. It follows that $c(x, y)=n_{\Pi(\delta)^{-1}} / n_{\Pi(\delta)}=n_{\delta^{-1}} / n_{\delta}$ for each $(x, y) \in \mathcal{D}_{\delta}^{\prime} \backslash \mathcal{R}_{1}$.

By definition, $\operatorname{Ker}(c)$ coincides with $\bigcup\left\{\mathcal{C}_{\lambda}: n_{\lambda}=n_{\lambda^{-1}}\right\} \cup\left(\mathcal{R} \backslash \bigcup_{\lambda \in \Lambda} \mathcal{C}_{\lambda}^{\prime}\right)$. In particular, this is an intermediate equivalence subrelation for $N_{\mathcal{R}}(\mathcal{S}) \subseteq \operatorname{Comm}_{\mathcal{R}}(\mathcal{S})$ up to a null set.

We note that if $\mathcal{S} \subseteq \mathcal{R}$ comes from the crossed product of outer actions $H \subseteq G$ on an equivalence subrelation $\mathcal{P}$ such that $H$ is commensurable in $G$, then $c$ is defined by

$$
c(x, g y)=\frac{\left[H: H \cap g H g^{-1}\right]}{\left[H: H \cap g^{-1} H g\right]} \quad((x, y) \in \mathcal{P}) .
$$

So the 1-cocycle $c$ is a natural generalization of the group homomorphism canonically associated with a Hecke pair of groups (cf. 4, Proposition 4], 15, Proposition 3.6], [22, Proposition 2.2]).

\section{§9.2. Hecke pairs of measured equivalence relations}

In Section 6, we studied the restriction of the $*$-isomorphism $\Phi$ to the relative commutant $A_{1} \cap B^{\prime}$. Our immediate purpose in this subsection is to show that

(i) this restricted $*$-isomorphism $\left.\Phi\right|_{A_{1} \cap B^{\prime}}$ can be constructed directly from the "groupoid" information on the pair $(\mathcal{R}, \mathcal{S})$ only, without passing to the basic construction or the factor $W^{*}\left(I_{\sigma} \times \mathcal{R}, \omega^{\sigma}\right)$;

(ii) discreteness of the inclusion $W^{*}(\mathcal{S}, \omega) \subseteq W^{*}(\mathcal{R}, \omega)$, or equivalently, equality $\operatorname{Comm}_{\mathcal{R}}(\mathcal{S})=\mathcal{R}$ can be rephrased from the viewpoint stated above (Proposition 9.6).

For a not necessarily ergodic $\mathcal{R}$ and a Borel subrelation $\mathcal{S}$ of $\mathcal{R}$, we denote by $L^{\infty}(\mathcal{S} \backslash \mathcal{R} / \mathcal{S})$ the set of all functions $Z \in L^{\infty}(\mathcal{R}, \nu)$ satisfying

$$
Z(x, z)=Z(y, z), \quad Z(z, x)=Z(z, y) \quad \text { for a.e. }(x, y) \in \mathcal{S} \text { and all } z \in \mathcal{R}(x) .
$$


Since $L^{\infty}(\mathcal{S} \backslash \mathcal{R} / \mathcal{S})=L^{\infty}(\mathcal{R}, \nu) \cap W^{*}(\mathcal{S})^{\prime}, L^{\infty}(\mathcal{S} \backslash \mathcal{R} / \mathcal{S})$ is a von Neumann subalgebra of $L^{\infty}(\mathcal{R}, \nu)$. Every element in $L^{\infty}(\mathcal{S} \backslash \mathcal{R} / \mathcal{S})$ is said to be a two-sided $\mathcal{S}$ invariant function.

From now on, we assume as before that $\mathcal{R}$ is ergodic. We then choose choice functions $\left\{\psi_{i}\right\}_{i \in I}$ for the pair $\mathcal{S} \subseteq \mathcal{R}$ and consider the associated index cocycle $\sigma$. Here $I=\{0,1, \ldots, N\}(N$ could be $\infty)$, and we assume that $\psi_{0}=\mathrm{id}_{X}$.

We consider the set of all functions $F$ in $L^{\infty}\left(I \times X, \mu_{c} \times \mu\right)$ satisfying

$$
F(i, x)=F(\sigma(y, x)(i), y) \quad \text { for a.e. }(x, y) \in \mathcal{S} \text { and all } i \in I,
$$

where $\mu_{c}$ is the counting measure on $I$. We note that this is nothing but $L^{\infty}(I \times X)^{\mathcal{S}}$, defined in Section 3. Because $L^{\infty}(I \times X)^{\mathcal{S}}$ is realized as the "commuting algebra" in $\ell^{\infty}(I) \otimes D$ with respect to a certain groupoid representation of $\mathcal{S}$ on the Hilbert bundle $\left\{\ell^{2}(I) \otimes \ell^{2}\left(\mathcal{R}^{x}\right)\right\}_{x \in X}$, it is a von Neumann algebra.

For any $Z \in L^{\infty}(\mathcal{S} \backslash \mathcal{R} / \mathcal{S})$, define a Borel function $\Phi(Z)$ in $L^{\infty}(I \times X)$ by

$$
\Phi(Z)(i, x):=Z\left(x, \psi_{i}(x)\right) \quad((i, x) \in I \times X) .
$$

Notice that the map $\Phi$ is nothing but the $*$-isomorphism discussed in Sections 3 and 6. Moreover, we have the following:

Proposition 9.5. The map $\Phi$ defined above is a $*$-isomorphism from $L^{\infty}(\mathcal{S} \backslash \mathcal{R} / \mathcal{S})$ onto $L^{\infty}(I \times X)^{\mathcal{S}}$. In particular, the von Neumann algebraic structure of $L^{\infty}(I \times X)^{\mathcal{S}}$ does not depend on the choice of choice functions $\left\{\psi_{i}\right\}_{i \in I}$.

Proof. It is easy to see that $\Phi$ is an injective $*$-homomorphism into $L^{\infty}(I \times X)$.

Let $Z \in L^{\infty}(\mathcal{S} \backslash \mathcal{R} / \mathcal{S})$. So there exists a $\nu$-null set $\mathcal{N}$ in $\mathcal{S}$ such that

$$
Z(x, z)=Z(y, z), \quad Z(z, x)=Z(z, y) \quad \text { for all }(x, y) \in \mathcal{S} \backslash \mathcal{N} \text { and all } z \in \mathcal{R}(x)
$$

Fix any $i \in I$. For each $j \in I$, put $\mathcal{S}_{j}:=\{(x, y) \in \mathcal{S}: \sigma(x, y)(i)=j\}$. Since $\left.\mathcal{S}_{j}=\left(\left.\left(\psi_{j} \times \psi_{i}\right)\right|_{\mathcal{S}}\right)\right)^{-1}(\mathcal{S}), \mathcal{S}_{j}$ is Borel for all $j \in I$. By the nonsingularity of $\psi_{i}$ 's, all the subsets $\left.\left(\left.\left(\psi_{j} \times \psi_{i}\right)\right|_{\mathcal{S}_{j}}\right)\right)^{-1}(\mathcal{N})(i, j \in I)$ are $\nu$-null, so that $\left.\overline{\mathcal{N}}:=\bigcup_{i, j \in I}\left(\left.\left(\psi_{j} \times \psi_{i}\right)\right|_{\mathcal{S}_{j}}\right)\right)^{-1}(\mathcal{N})$ is also $\nu$-null. Then we clearly have

$$
\left(\psi_{i}(x), \psi_{\sigma(y, x)(i)}(y)\right) \in \mathcal{S} \backslash \mathcal{N} \quad \text { for all }(x, y) \in \mathcal{S} \backslash \overline{\mathcal{N}} \text { and all } i \in I \text {. }
$$

Hence, for any $(x, y) \in \mathcal{S} \backslash \overline{\mathcal{N}}$ and any $i \in I$,

$$
\begin{aligned}
\Phi(Z)(\sigma(y, x)(i), y) & =Z\left(y, \psi_{\sigma(y, x)(i)}(y)\right) \\
& =Z\left(y, \psi_{i}(x)\right) \quad\left(\operatorname{as}\left(\psi_{i}(x), \psi_{\sigma(y, x)(i)}(y)\right) \in \mathcal{S} \backslash \mathcal{N}\right)
\end{aligned}
$$




$$
\begin{aligned}
& =Z\left(x, \psi_{i}(x)\right) \quad(\text { as }(x, y) \in \mathcal{S} \backslash \mathcal{N}) \\
& =\Phi(Z)(i, x) .
\end{aligned}
$$

Thus $\Phi(Z)$ belongs to $L^{\infty}(I \times X)^{\mathcal{S}}$.

To show the surjectivity of $\Phi$, take an arbitrary $F \in L^{\infty}(I \times X)^{\mathcal{S}}$. Then there is a $\nu$-null subset $\mathcal{N}_{1}$ of $\mathcal{S}$ such that

$$
F(i, x)=F(\sigma(y, x)(i), y) \quad \text { for all }(x, y) \in \mathcal{S} \backslash \mathcal{N}_{1} \text { and all } i \in I .
$$

It follows that there exists a $\mu$-null subset $N_{1}$ of $X$ such that $r^{-1}(x) \cap \mathcal{N}_{1}=\emptyset$ for all $x \in N_{1}^{c}$. Here, we may and do assume that $N_{1}$ is $\mathcal{R}$-invariant.

On the other hand, there is a $\mu$-null subset $N_{2}$ of $X$ such that $\mathcal{R}(x)=$ $\bigsqcup_{i \in I} \mathcal{S}\left(\psi_{i}(x)\right)$ for all $x \in N_{2}^{c}$. Once again, we may assume that $N_{2}$ is $\mathcal{R}$-invariant. Put $N_{0}:=N_{1} \cup N_{2}$ and $\mathcal{R}_{0}:=\mathcal{R} \cap\left(N_{0}^{c} \times N_{0}^{c}\right)$. Clearly, $\mathcal{R} \backslash \mathcal{R}_{0}$ is $\nu$-null. We then define a Borel function $Z_{0} \in L^{\infty}(\mathcal{R}, \nu)$ by

$$
Z_{0}(x, y):= \begin{cases}F(i, x) & \text { if }(x, y) \in \mathcal{R}_{0} \text { and } y \in \mathcal{S}\left(\psi_{i}(x)\right), \\ 0 & \text { if }(x, y) \in \mathcal{R} \backslash \mathcal{R}_{0} .\end{cases}
$$

Let $(x, y) \in \mathcal{S} \backslash\left(\mathcal{R} \backslash \mathcal{R}_{0}\right)=\mathcal{S} \cap \mathcal{R}_{0}$ and $z \in \mathcal{R}(x)$. There is a unique $i \in I$ such that $z \in \mathcal{S}\left(\psi_{i}(x)\right)$. Then

$$
Z_{0}(x, z)=F(i, x)=F(\sigma(y, x)(i), y) \quad\left(\text { as }(x, y) \in \mathcal{S} \backslash \mathcal{N}_{1}\right) .
$$

Note that $(y, z)$ too belongs to $\mathcal{R}_{0}$. Since $\left(\psi_{\sigma(y, x)(i)}(y), \psi_{i}(x)\right) \in \mathcal{S}$, it follows that $\left(z, \psi_{\sigma(y, x)(i)}(y)\right) \in \mathcal{S}$. So, by definition, $Z_{0}(y, z)=F(\sigma(y, x)(i), y)$. Hence $Z_{0}(x, z)=Z_{0}(y, z)$. Meanwhile, because $(z, x) \in \mathcal{R}_{0}$, there exists a unique $j \in I$ such that $x \in \mathcal{S}\left(\psi_{j}(z)\right)$. Thus $Z_{0}(z, x)=F(j, z)$. Since $(x, y) \in \mathcal{S}$, we have $y \in$ $\mathcal{S}\left(\psi_{j}(z)\right)$, which implies $Z_{0}(z, y)=F(j, z)$. So $Z_{0}(z, x)=Z_{0}(z, y)$. Therefore, $Z_{0} \in L^{\infty}(\mathcal{S} \backslash \mathcal{R} / \mathcal{S})$. By the definition of $Z_{0}$, if $x \in N_{0}^{c}$, then, for any $i \in I$,

$$
\Phi\left(Z_{0}\right)(i, x)=Z_{0}\left(x, \psi_{i}(x)\right)=F(i, x) .
$$

This shows that $\Phi\left(Z_{0}\right)=F$.

We have the normal embedding of $L^{\infty}(X, \mu)$ into $L^{\infty}(\mathcal{R}, \nu)$ via the mapping $f \in L^{\infty}(X, \mu) \mapsto f \circ r \in L^{\infty}(\mathcal{R}, \nu)$. We will freely identify $L^{\infty}(X, \mu)$ with the image under this embedding and simply write $f$ for $f \circ r$. If $L^{\infty}(X, \mu)^{\mathcal{S}}$ is the algebra of all $\mathcal{S}$-invariant functions $f \in L^{\infty}(X, \mu)$ (i.e., $f(x)=f(y)$ for a.e. $\left.(x, y) \in \mathcal{S}\right)$, then it is clearly contained in $L^{\infty}(\mathcal{S} \backslash \mathcal{R} / \mathcal{S})$ and we have $\Phi\left(L^{\infty}(X, \mu)^{\mathcal{S}}\right)=\mathbb{C} \otimes L^{\infty}(X, \mu)^{\mathcal{S}}$.

We denote by $\tau_{0}$ the faithful normal semifinite trace on $\ell^{\infty}(I)$ given by $\tau_{0}(f):=\sum_{i \in I} f(i)$ for any $f \in \ell^{\infty}(I)_{+}$. Then $\tau_{0} \otimes \operatorname{id}_{L^{\infty}(X, \mu)}$ is a faithful normal semifinite operator valued weight from $L^{\infty}(I \times X)$ onto the von Neumann 
subalgebra $\mathbb{C} \otimes L^{\infty}(X, \mu) \cong L^{\infty}(X, \mu)$. If $F \in\left(L^{\infty}(I \times X)^{\mathcal{S}}\right)_{+}$, then we have $\left(\tau_{0} \otimes \operatorname{id}_{L^{\infty}(X, \mu)}\right)(F)(x)=\sum_{i \in I} F(i, x)$. Thus, for a.e. $(x, y) \in \mathcal{S}$,

$$
\begin{aligned}
\left(\tau_{0} \otimes \operatorname{id}_{L^{\infty}(X, \mu)}\right)(F)(y) & =\sum_{i \in I} F(i, y)=\sum_{i \in I} F(\sigma(y, x)(i), y) \\
& =\sum_{i \in I} F(i, x)=\left(\tau_{0} \otimes \operatorname{id}_{L^{\infty}(X, \mu)}\right)(F)(x) .
\end{aligned}
$$

This means that $\left(\tau_{0} \otimes \operatorname{id}_{L^{\infty}(X, \mu)}\right)(F)$ belongs to the extended positive part of $L^{\infty}(X, \mu)^{\mathcal{S}}$. Therefore, the restriction $T_{\mathcal{S}}$ of $\tau_{0} \otimes \operatorname{id}_{L^{\infty}(X, \mu)}$ to $L^{\infty}(I \times X)^{\mathcal{S}}$ is a faithful normal operator valued weight onto $L^{\infty}(X, \mu)^{\mathcal{S}}$.

From this point on, we assume that the subrelation $\mathcal{S}$ is also ergodic. So we have $L^{\infty}(X, \mu)^{\mathcal{S}}=\mathbb{C}$. Hence $T_{\mathcal{S}}$ is regarded as a faithful normal weight on $L^{\infty}(I \times X)^{\mathcal{S}}$, to be denoted by $\Omega$ in what follows. Therefore, $\Omega$ is given by

$$
\Omega(F)=\int_{X} \sum_{i \in I} F(i, x) d m(x) \quad\left(\forall F \in\left(L^{\infty}(I \times X)^{\mathcal{S}}\right)_{+}\right)
$$

for any probability measure $m$ on $X$ equivalent to $\mu$.

On the other hand, for each $\rho \in[\mathcal{R}]_{*}$ which satisfies $\mu(\operatorname{Dom}(\rho))>0$, we define a nonzero projection $Z_{\rho}$ in $L^{\infty}(\mathcal{R}, \nu)$ by

$$
Z_{\rho}(x, y)= \begin{cases}1, & y \in \mathcal{S}(\rho(\mathcal{S}(x))) \\ 0, & \text { otherwise }\end{cases}
$$

It is easy to check that $Z_{\rho} \in L^{\infty}(\mathcal{S} \backslash \mathcal{R} / \mathcal{S})$. Moreover, by the definition of the index,

$$
\Omega\left(\Phi\left(Z_{\rho}\right)\right)=\operatorname{Ind}(\rho) .
$$

So we have the following:

Proposition 9.6. The following are equivalent:

(1) The commensurability groupoid $\operatorname{Comm}_{\mathcal{R}}(\mathcal{S})$ equals $\mathcal{R}$ up to a $\nu$-null set.

(2) The weight $\Omega$ is semifinite.

Proof. (1) $\Rightarrow(2):$ Set $\mathcal{C}_{\lambda, n}:=\left\{(x, y) \in \mathcal{R}: \exists z \in X,(x, z) \in \mathcal{S}\right.$ and $\left(\psi_{\lambda, n}(z), y\right)$ $\in \mathcal{S}\}$ as before. As noted previously, $\mathcal{C}_{\lambda, n}$ is equal to $\mathcal{C}_{\lambda, m}$ up to a null set. We also define $\mathcal{C}_{\lambda}:=\mathcal{C}_{\lambda, 1}$. By definition, each $\chi_{\mathcal{C}_{\lambda}}$ belongs to $L^{\infty}(\mathcal{S} \backslash \mathcal{R} / \mathcal{S})$. We note that $\left\{\mathcal{C}_{\lambda}\right\}_{\lambda \in \Lambda}$ is a measurable partition of $\mathcal{R}$ up to a null set, because it satisfies $\sum_{\lambda \in \Lambda} \chi_{\mathcal{C}_{\lambda}}=1$ (see the proof of [2, Theorem 3.8]). Thus $\left\{F^{\lambda}:=\Phi\left(\chi_{\mathcal{C}_{\lambda}}\right)\right\}_{\lambda \in \Lambda}$ in 
turn is the set of projections in $L^{\infty}(I \times X)^{\mathcal{S}}$ satisfying $\sum_{\lambda \in \Lambda} F^{\lambda}=1$. Moreover,

$$
\begin{aligned}
\Omega\left(F^{\lambda}\right) & =\int_{X} \sum_{\lambda^{\prime} \in \Lambda} \sum_{n=1}^{n_{\lambda^{\prime}}} \chi_{\mathcal{C}_{\lambda}}\left(x, \psi_{\lambda^{\prime}, n}(x)\right) d \mu(x)=\int_{X} \sum_{n=1}^{n_{\lambda}} \chi_{\mathcal{C}_{\lambda}}\left(x, \psi_{\lambda, n}(x)\right) d \mu(x)=n_{\lambda} \\
& <\infty
\end{aligned}
$$

It follows that the trace $\Omega$ is semifinite.

$(2) \Rightarrow(1)$ : Suppose that $\operatorname{Comm}_{\mathcal{R}}(\mathcal{S})$ is not $\nu$-conull in $\mathcal{R}$. Then there exists $\rho \in[\mathcal{R}]_{*}$ such that $\operatorname{Ind}\left(\left.\rho\right|_{E}\right)$ is equal to $\infty$ for each nonnull subset $E$ of $\operatorname{Dom}(\rho)$.

Fix a nonzero projection $Z \leq Z_{\rho}$ in $L^{\infty}(\mathcal{S} \backslash \mathcal{R} / \mathcal{S})$. By the definition of $Z_{\rho}$, there exist $\theta_{1}, \theta_{2} \in[\mathcal{S}]_{*}$ such that $\mu\left(\operatorname{Dom}\left(\theta_{2}\right)\right)>0, \operatorname{Dom}\left(\theta_{1}\right) \subseteq \operatorname{Im}(\rho), \operatorname{Im}\left(\rho_{2}\right) \subseteq \operatorname{Dom}(\rho)$ and $\chi_{\Gamma\left(\theta_{1} \circ \rho \circ \theta_{2}\right)}$ is dominated by $Z$. Since $Z \in L^{\infty}(\mathcal{S} \backslash \mathcal{R} / \mathcal{S})$, we get $\chi_{\left.\rho\right|_{\operatorname{Im}\left(\theta_{2}\right)}} \leq Z$ and $Z_{\left.\rho\right|_{\operatorname{Im}\left(\theta_{2}\right)}} \leq Z$. It follows that

$$
\Omega(Z) \geq \Omega\left(Z_{\left.\rho\right|_{\operatorname{Im}\left(\theta_{2}\right)}}\right)=\operatorname{Ind}\left(\left.\rho\right|_{\operatorname{Im}\left(\theta_{2}\right)}\right)=\infty .
$$

Hence $\Omega$ is not semifinite.

In what follows, we assume that $\operatorname{Comm}_{\mathcal{R}}(\mathcal{S})$ equals $\mathcal{R}$ up to a $\nu$-null set. Let us refer to this situation by saying that $(\mathcal{R}, \mathcal{S})$ is a Hecke pair. We fix the choice functions $\left\{\psi_{\lambda, n}\right\}_{(\lambda, n) \in I}$ for $\mathcal{S} \subseteq \mathcal{R}$ introduced in Section 8 . We also retain the symbols $\mathcal{C}_{\lambda}, F^{\lambda}$ used there. By (the proof of) Proposition 9.6. we know that $\Omega$ is semifinite, and that the projections $F^{\lambda}(\lambda \in \Lambda)$ all belong to $\mathfrak{n}_{\Omega}$.

Fix any $\lambda \in \Lambda$. Suppose that there exists a $\rho \in[\mathcal{R}]_{*}$ such that $0 \neq \chi_{\Gamma(\rho)} \leq \chi_{\mathcal{C}_{\lambda}}$. Without any loss of generality, we may and do assume that $\Gamma(\rho) \subseteq \mathcal{C}_{\lambda}=\mathcal{C}_{\lambda, 1}$. Hence $\rho(x) \in \mathcal{S}\left(\psi_{\lambda, 1}(\mathcal{S}(x))\right)$ for all $x \in \operatorname{Dom}(\rho)$. Choose a countable set $\left\{h_{n}\right\}_{n \in S}$ in $[\mathcal{S}]$ so that $\mathcal{S}=\left\{\left(x, h_{n}(x)\right): x \in X, n \in S\right\}$. For a pair $(n, m) \in S \times S$, define a Borel map $\varphi_{n, m}: \operatorname{Dom}(\rho) \rightarrow X$ by $\varphi_{n, m}(x):=h_{m}\left(\psi_{\lambda, 1}\left(h_{n}(x)\right)\right)$. We also define a Borel subset $A_{n, m}$ of $\operatorname{Dom}(\rho)$ by $A_{n, m}:=\left\{x \in \operatorname{Dom}(\rho): \varphi_{n, m}(x)=\rho(x)\right\}$. Since $\rho(x) \in \mathcal{S}\left(\psi_{\lambda, 1}(\mathcal{S}(x))\right)$ for all $x \in \operatorname{Dom}(\rho)$, we have $\bigcup_{(n, m)} A_{n, m}=\operatorname{Dom}(\rho)$. Choose a pair $\left(n_{0}, m_{0}\right)$ such that $\mu\left(A_{n_{0}, m_{0}}\right)>0$ and put $A:=A_{n_{0}, m_{0}}, B:=h_{n_{0}}(A)$. By 2, Theorem 3.8(3)], there exists a $\mu$-null set $N$ of $X$ such that $\mathcal{S}\left(\left.\psi_{\lambda, 1}\right|_{B}(\mathcal{S}(x))\right)=$ $\mathcal{S}\left(\psi_{\lambda, 1}(\mathcal{S}(x))\right)$ for all $x \in N^{c}$. Let $(x, y) \in \mathcal{C}_{\lambda, 1} \cap\left(N^{c} \times N^{c}\right)$. So there is a $z \in X$ such that $(x, z) \in \mathcal{S}$ and $\left(\psi_{\lambda, 1}(z), y\right) \in \mathcal{S}$. Since $y \in \mathcal{S}\left(\psi_{\lambda, 1}(\mathcal{S}(x))\right)=$ $\mathcal{S}\left(\left.\psi_{\lambda, 1}\right|_{B}(\mathcal{S}(x))\right)$, there is a $b \in B$ such that $(x, b) \in \mathcal{S}$ and $\left(\psi_{\lambda, 1}(b), y\right) \in \mathcal{S}$. With $a:=h_{n_{0}}^{-1}(b) \in \operatorname{Dom}(\rho)$, we have $h_{m_{0}}\left(\psi_{\lambda, 1}\left(h_{n_{0}}(a)\right)\right)=\varphi_{n_{0}, m_{0}}(a)=\rho(a)$. Thus $(x, a)=(x, b)(b, a) \in \mathcal{S}$ and $(\rho(a), y)=\left(\rho(a), \psi_{\lambda, 1}(b)\right)\left(\psi_{\lambda, 1}(b), y\right) \in \mathcal{S}$. Hence $(x, y)$ belongs to the Borel set

$$
\mathcal{E}_{\rho}:=\{(u, v) \in \mathcal{R}: \exists w \in \operatorname{Dom}(\rho),(u, w) \in \mathcal{S} \text { and }(\rho(w), v) \in \mathcal{S}\},
$$


which is clearly a two-sided $\mathcal{S}$-invariant set. This in turn implies that $\chi_{\mathcal{C}_{\lambda}} \leq \chi_{\mathcal{E}_{\rho}}$ as functions in $L^{\infty}(\mathcal{S} \backslash \mathcal{R} / \mathcal{S})$. The discussion in this paragraph immediately shows that the projection $\chi_{\mathcal{C}_{\lambda}}$ is minimal in $L^{\infty}(\mathcal{S} \backslash \mathcal{R} / \mathcal{S})$ for each $\lambda \in \Lambda$. Therefore the abelian von Neumann algebra $L^{\infty}\left(\mathcal{S} \backslash \mathcal{R} / \mathcal{S}\right.$ ) (or, equivalently, $L^{\infty}(I \times X)^{\mathcal{S}}$ ) is atomic and generated by the minimal projections $\left\{\chi_{\mathcal{C}_{\lambda}}\right\}_{\lambda \in \Lambda}$ (resp. $\left\{F^{\lambda}\right\}_{\lambda \in \Lambda}$ ), when $(\mathcal{R}, \mathcal{S})$ is a Hecke pair.

Lemma 9.7. The map $\Xi: I \times X \rightarrow \Lambda$ defined by

$$
\Xi((\lambda, n), x):=\lambda \quad((\lambda, n) \in I, x \in X)
$$

is an $\mathcal{S}$-factor map.

Proof. It is clear that $\Xi$ is Borel. Let $f \in \ell^{\infty}(\Lambda)$ and $(x, y) \in \mathcal{S}$. Put $\left(\lambda^{\prime}, n^{\prime}\right):=$ $\sigma(y, x)(\lambda, n)$. Then $\left(\psi_{\lambda^{\prime}, n^{\prime}}(y), \psi_{\lambda, n}(x)\right) \in \mathcal{S}$. By definition, $\left(x, \psi_{\lambda, n}(x)\right) \in \mathcal{C}_{\lambda}$ and $\left(y, \psi_{\lambda^{\prime}, n^{\prime}}(y)\right) \in \mathcal{C}_{\lambda^{\prime}}$. We also have

$$
\left(x, \psi_{\lambda, n}(x)\right)=(x, y)\left(y, \psi_{\lambda^{\prime}, n^{\prime}}(y)\right)\left(\psi_{\lambda^{\prime}, n^{\prime}}(y), \psi_{\lambda, n}(x)\right) .
$$

Since both $(x, y)$ and $\left(\psi_{\lambda^{\prime}, n^{\prime}}(y), \psi_{\lambda, n}(x)\right)$ belong to $\mathcal{S}$, it follows that $\lambda^{\prime}=\lambda$. So $\Xi(\sigma(y, x)(\lambda, n), z)=\lambda$ for any $z \in X$. Hence

$$
f \circ \Xi(\sigma(y, x)(\lambda, n), y)=f(\lambda)=f \circ \Xi((\lambda, n), x) .
$$

This shows that $f \circ \Xi$ is $\mathcal{S}$-invariant.

Conversely, suppose that $F$ is a bounded $\mathcal{S}$-invariant Borel function on $I \times X$. Since $L^{\infty}(I \times X)^{\mathcal{S}}$ is generated by the minimal projections $\left\{F^{\lambda}\right\}_{\lambda \in \Lambda}, F$ has the form $F=\sum_{\lambda \in \Lambda} c_{\lambda} F^{\lambda}$ for some $c_{\lambda} \in \mathbb{C}(\lambda \in \Lambda)$, where the sum is meant in the strong operator topology. Put $f(\lambda):=c_{\lambda}$, which is a bounded function on $\Lambda$. Then, for any $(\lambda, n) \in I$,

$$
F((\lambda, n), x)=c_{\lambda}=f(\lambda)=f \circ \Xi((\lambda, n), x) .
$$

Hence $F=f \circ \Xi$. This completes the proof.

Thanks to Lemma 9.7, the factor map $\Xi$ induces a $*$-isomorphism $\Xi^{*}$ from $\ell^{\infty}(\Lambda)$ onto $L^{\infty}(I \times X)^{\mathcal{S}}$ given by $\Xi^{*}(f):=f \circ \Xi$, where $f \in \ell^{\infty}(\Lambda)$. Note that $\Xi^{*}\left(\delta_{\lambda}\right)=F^{\lambda}$ for all $\lambda \in \Lambda$. For any $f \in \ell^{\infty}(\Lambda)_{+}$, with $F=\Xi^{*}(f)$, we then have

$$
\begin{aligned}
\Omega(F) & =\int_{X} \sum_{(\lambda, n) \in I} F((\lambda, n), x) d \mu(x)=\int_{X} \sum_{(\lambda, n) \in I} f \circ \Xi((\lambda, n), x) d \mu(x) \\
& =\sum_{\lambda \in \Lambda} f(\lambda) n_{\lambda} .
\end{aligned}
$$


For any $F \in L^{\infty}(I \times X)^{\mathcal{S}}$, define a Borel function $F^{\sharp}$ on $I \times X$ by

$$
F^{\sharp}(i, x):=\overline{F\left(\sigma\left(\psi_{i}(x), x\right)(0), \psi_{i}(x)\right)} \quad((i, x) \in I \times X) .
$$

It is obvious that $F^{\sharp} \in L^{\infty}(I \times X)$. By definition, there exists a $\mu$-null subset $N_{0}$ of $X$ such that, setting $\mathcal{S}_{0}:=\mathcal{S} \cap\left(N_{0}^{c} \times N_{0}^{c}\right)$, we have $F(\sigma(y, x)(i), y)=F(i, x)$ for all $(x, y) \in \mathcal{S}_{0}$ and all $i \in I$. Put $N:=\bigcup_{i, j \in I} \psi_{j}^{-1}\left(\psi_{i}^{-1}\left(N_{0}\right)\right)$, which is again a $\mu$-null set. Set $\mathcal{S}_{1}:=\mathcal{S} \cap\left(N^{c} \times N^{c}\right)$. Let $(x, y) \in \mathcal{S}_{1}$. We have $\left(\psi_{\sigma(y, x)(i)}(y), \psi_{i}(x)\right) \in \mathcal{S}_{0}$. Hence

$$
\begin{aligned}
F^{\sharp}(\sigma(y, x)(i), y) & =\overline{F\left(\sigma\left(\psi_{\sigma(y, x)(i)}(y), y\right)(0), \psi_{\sigma(y, x)(i)}(y)\right)} \\
& =\overline{F\left(\sigma\left(\psi_{\sigma(y, x)(i)}(y), \psi_{i}(x)\right)\left(\sigma\left(\psi_{i}(x), x\right)(\sigma(x, y)(0))\right), \psi_{\sigma(y, x)(i)}(y)\right)} \\
& =\overline{F\left(\sigma\left(\psi_{\sigma(y, x)(i)}(y), \psi_{i}(x)\right)\left(\sigma\left(\psi_{i}(x), x\right)(0)\right), \psi_{\sigma(y, x)(i)}(y)\right)} \\
& =\overline{F\left(\sigma\left(\psi_{i}(x), x\right)(0), \psi_{i}(x)\right)}=F^{\sharp}(i, x) .
\end{aligned}
$$

It follows that $F^{\sharp}$ too belongs to $L^{\infty}(I \times X)^{\mathcal{S}}$. Moreover,

$$
\begin{aligned}
\left(F^{\sharp}\right)^{\sharp}(i, x) & =\overline{F^{\sharp}\left(\sigma\left(\psi_{i}(x), x\right)(0), \psi_{i}(x)\right)} \\
& =F\left(\sigma\left(\psi_{\sigma\left(\psi_{i}(x), x\right)(0)}\left(\psi_{i}(x)\right), \psi_{i}(x)\right)(0), \psi_{\sigma\left(\psi_{i}(x), x\right)(0)}\left(\psi_{i}(x)\right)\right) \\
& =F\left(\sigma\left(\psi_{\sigma\left(\psi_{i}(x), x\right)(0)}\left(\psi_{i}(x)\right), x\right)\left(\sigma\left(x, \psi_{i}(x)\right)(0)\right), \psi_{\sigma\left(\psi_{i}(x), x\right)(0)}\left(\psi_{i}(x)\right)\right) \\
& =F\left(\sigma\left(x, \psi_{i}(x)\right)(0), x\right) \quad\left(\text { as }\left(\psi_{\sigma\left(\psi_{i}(x), x\right)(0)}\left(\psi_{i}(x)\right), x\right) \in \mathcal{S}_{0}\right) \\
& =F(i, x) .
\end{aligned}
$$

Thus $\left(F^{\sharp}\right)^{\sharp}=F$. Clearly, the mapping $F \mapsto F^{\sharp}$ is conjugate-linear.

Lemma 9.8. For any $Z \in L^{\infty}(\mathcal{S} \backslash \mathcal{R} / \mathcal{S})$, define $Z^{\vee} \in L^{\infty}(\mathcal{S} \backslash \mathcal{R} / \mathcal{S})$ by $Z^{\vee}(x, y):=$ $Z(y, x)$. Then $\Phi\left(\bar{Z}^{\vee}\right)=\Phi(Z)^{\sharp}$ for all $Z \in L^{\infty}(\mathcal{S} \backslash \mathcal{R} / \mathcal{S})$. In particular, $\left(F^{\lambda}\right)^{\sharp}=$ $F^{\lambda^{-1}}$ for all $\lambda \in \Lambda$.

Proof. Let $Z \in L^{\infty}(\mathcal{S} \backslash \mathcal{R} / \mathcal{S})$. There exists a $\mu$-null subset $N_{1}$ of $X$ such that

$$
\begin{aligned}
& Z(x, z)=Z(y, z), \\
& Z(z, x)=Z(z, y) \quad \text { for all }(x, y) \in \mathcal{S}_{0}:=\mathcal{S} \cap\left(N_{1}^{c} \times N_{1}^{c}\right) \text { and all } z \in \mathcal{R}(x) .
\end{aligned}
$$

Put $N_{1}:=\bigcup_{i, j \in I} \psi_{j}^{-1}\left(\psi_{i}^{-1}\left(N_{1}\right)\right)$. Let $x \in N_{1}^{c}$ and $i \in I$. Then

$$
\begin{aligned}
\Phi\left(\bar{Z}^{\vee}\right)(i, x) & =\overline{Z\left(\psi_{i}(x), x\right)} \\
& =\overline{Z\left(\psi_{i}(x), \psi_{\sigma\left(\psi_{i}(x), x\right)(0)}\left(\psi_{i}(x)\right)\right.} \quad\left(\operatorname{as}\left(x, \psi_{\sigma\left(\psi_{i}(x), x\right)(0)}\left(\psi_{i}(x)\right)\right) \in \mathcal{S}_{0}\right) \\
& =\overline{\Phi(Z)\left(\sigma\left(\psi_{i}(x), x\right)(0), \psi_{i}(x)\right)} \\
& =\Phi(Z)^{\sharp}(i, x) .
\end{aligned}
$$

Thus $\Phi\left(\bar{Z}^{\vee}\right)=\Phi(Z)^{\sharp}$. For the last assertion, just notice that $\left(\chi_{\mathcal{C}_{\lambda}}\right)^{\vee}=\chi_{\mathcal{C}_{\lambda-1}}$. 
Lemma 9.9. Let $f \in \ell^{\infty}(\Lambda)_{+}$and $F=\Xi^{*}(f)$. Then

$$
\Omega\left(F^{\sharp}\right)=\sum_{\lambda \in \Lambda} \overline{f\left(\lambda^{-1}\right)} n_{\lambda} .
$$

Proof. Let $(\lambda, n) \in I$ and $x \in X$. Put $\left(\lambda^{\prime}, n^{\prime}\right):=\sigma\left(\psi_{\lambda, n}(x), x\right)(0)$. Then we have $\left(\psi_{\lambda^{\prime}, n^{\prime}}\left(\psi_{\lambda, n}(x)\right), x\right) \in \mathcal{S}$. But this means that $\left(\psi_{\lambda, n}(x), x\right)$ is in $\mathcal{C}_{\lambda^{\prime}}$. Since $\left(x, \psi_{\lambda, n}(x)\right) \in \mathcal{C}_{\lambda}$, it follows that $\lambda^{\prime}=\lambda^{-1}$. Hence $\Xi\left(\sigma\left(\psi_{\lambda, n}(x), x\right)(0), z\right)=\lambda^{-1}$ for any $z \in X$. Thus,

$$
\begin{aligned}
F^{\sharp}((\lambda, n), x) & =\overline{\Xi^{*}(f)\left(\sigma\left(\psi_{\lambda, n}(x), x\right)(0), \psi_{\lambda, n}(x)\right)}=\overline{f \circ \Xi\left(\sigma\left(\psi_{\lambda, n}(x), x\right)(0), \psi_{\lambda, n}(x)\right)} \\
& =\overline{f\left(\lambda^{-1}\right)} .
\end{aligned}
$$

The assertion of the lemma now follows from 9.5).

We denote by $\mathcal{I}(\mathcal{R}, \mathcal{S})$ the set of all functions $F$ in $L^{\infty}(I \times X)^{\mathcal{S}}$ satisfying, with $F=\Xi^{*}(f)$,

$$
\|F\|_{1, \ell}:=\sum_{\lambda \in \Lambda}|f(\lambda)| n_{\lambda}<\infty \quad \text { and } \quad\|F\|_{1, r}:=\sum_{\lambda \in \Lambda}\left|f\left(\lambda^{-1}\right)\right| n_{\lambda}<\infty .
$$

We simply write $\mathcal{I}$ for $\mathcal{I}(\mathcal{R}, \mathcal{S})$ if there is no danger of confusion. By 9.5 and Lemma 9.9. $\mathcal{I}(\mathcal{R}, \mathcal{S})$ consists of the functions $F \in L^{\infty}(I \times X)^{\mathcal{S}}$ with $\Omega(|F|)<\infty$ and $\Omega\left(\left|F^{\sharp}\right|\right)<\infty$. It is then easy to see that $\mathcal{I}(\mathcal{R}, \mathcal{S})$ is a subspace of $L^{\infty}(I \times X)^{\mathcal{S}}$ which is closed under the $\sharp$-operation. Since $\Xi^{*}\left(\delta_{\lambda}\right)=F^{\lambda}$ for any $\lambda \in \Lambda$, the linear span $\mathcal{I}_{0}:=\mathcal{I}_{0}(\mathcal{R}, \mathcal{S})$ of $\left\{F^{\lambda}: \lambda \in \Lambda\right\}$ is contained in $\mathcal{I}(\mathcal{R}, \mathcal{S})$. Because $\mathcal{I}_{0}(\mathcal{R}, \mathcal{S})$ is $\sigma$-strongly* dense in $L^{\infty}(I \times X)^{\mathcal{S}}$, so is $\mathcal{I}(\mathcal{R}, \mathcal{S})$. If $F \in \mathfrak{n}_{\Omega}$ and $\Xi^{*}(f)=F$, then, by 9.5 ,

$$
\left\|\Lambda_{\Omega}(F)\right\|^{2}=\sum_{\lambda \in \Lambda}|f(\lambda)|^{2} n_{\lambda} .
$$

From this, we see that $\mathcal{I}(\mathcal{R}, \mathcal{S}) \subseteq \mathfrak{n}_{\Omega}$. Since $\mathcal{I}_{0}(\mathcal{R}, \mathcal{S})$ is a $\sigma$-strongly* dense $*$ subalgebra contained in $\mathfrak{n}_{\Omega}$, it follows that $\Lambda_{\Omega}\left(\mathcal{I}_{0}(\mathcal{R}, \mathcal{S})\right)$ is dense in the GNS Hilbert space $H_{\Omega}$. In particular, $\Lambda_{\Omega}(\mathcal{I}(\mathcal{R}, \mathcal{S}))$ is total in $H_{\Omega}$.

Let $F_{1}$ and $F_{2}$ be in $\mathcal{I}$. Define a Borel function $F_{1} * F_{2}$ on $I \times X$ by

$$
\left(F_{1} * F_{2}\right)(i, x):=\sum_{j \in I} F_{1}\left(\sigma\left(\psi_{j}(x), x\right)(i), \psi_{j}(x)\right) F_{2}(j, x) .
$$

We will show that this defines a product on $\mathcal{I}(\mathcal{R}, \mathcal{S})$ which makes it a $\sharp$-algebra. We first note that there exists a $\mu$-null subset $N_{0}$ of $X$ such that

$$
\begin{aligned}
F_{k}(\sigma(y, x)(i), y) & =F_{k}(i, x) \\
& \text { for all }(x, y) \in \mathcal{S}_{0}:=\mathcal{S} \cap\left(N_{0}^{c} \times N_{0}^{c}\right), i \in I \text { and } k=1,2 .
\end{aligned}
$$


Put $N_{1}:=\bigcup_{i, j \in I} \psi_{j}^{-1}\left(\psi_{i}^{-1}\left(N_{0}\right)\right)$ and $\mathcal{S}_{1}:=\mathcal{S} \cap\left(N_{1}^{c} \times N_{1}^{c}\right)$. Let $(x, y) \in \mathcal{S}_{1}$. Then, for any $i \in I$, we get

$$
\begin{aligned}
& \left(F_{1} * F_{2}\right)(\sigma(y, x)(i), y)=\sum_{j \in I} F_{1}\left(\sigma\left(\psi_{j}(y), y\right)(\sigma(y, x)(i)), \psi_{j}(y)\right) F_{2}(j, y) \\
& =\sum_{j \in I} F_{1}\left(\sigma\left(\psi_{j}(y), x\right)(i), \psi_{j}(y)\right) F_{2}(j, y) \\
& =\sum_{j \in I} F_{1}\left(\sigma\left(\psi_{\sigma(y, x)(j)}(y), x\right)(i), \psi_{\sigma(y, x)(j)}(y)\right) F_{2}(\sigma(y, x)(j), y) \quad(\text { as } I=\sigma(y, x)(I)) \\
& =\sum_{j \in I} F_{1}\left(\sigma\left(\psi_{\sigma(y, x)(j)}(y), x\right)(i), \psi_{\sigma(y, x)(j)}(y)\right) F_{2}(j, x) \quad\left(\operatorname{as}(x, y) \in \mathcal{S}_{1}\right) \\
& =\sum_{j \in I} F_{1}\left(\sigma\left(\psi_{\sigma(y, x))(j)}(y), \psi_{j}(x)\right)\left(\sigma\left(\psi_{j}(x), x\right)(i)\right), \psi_{\sigma(y, x)(j)}(y)\right) F_{2}(j, x) \\
& =\sum_{j \in I} F_{1}\left(\sigma\left(\psi_{j}(x), x\right)(i), \psi_{j}(x)\right) F_{2}(j, x) \quad\left(\operatorname{as}\left(\psi_{\sigma(y, x)(j)}(y), \psi_{j}(x)\right) \in \mathcal{S}_{0}\right) \\
& =\left(F_{1} * F_{2}\right)(i, x) .
\end{aligned}
$$

This shows that $F_{1} * F_{2}$ is $\mathcal{S}$-invariant. Moreover,

$$
\begin{aligned}
\left|\left(F_{1} * F_{2}\right)((\lambda, n), x)\right| & =\left|\sum_{\left(\lambda_{1}, n_{1}\right) \in I} F_{1}\left(\sigma\left(\psi_{\lambda_{1}, n_{1}}(x), x\right)(\lambda, n), \psi_{\lambda_{1}, n_{1}}(x)\right) F_{2}\left(\left(\lambda_{1}, n_{1}\right), x\right)\right| \\
& \leq \sum_{\left(\lambda_{1}, n_{1}\right) \in I}\left|F_{1}\left(\sigma\left(\psi_{\lambda_{1}, n_{1}}(x), x\right)(\lambda, n), \psi_{\lambda_{1}, n_{1}}(x)\right)\right|\left|F_{2}\left(\left(\lambda_{1}, n_{1}\right), x\right)\right| \\
& \leq\left\|F_{1}\right\|_{\infty} \sum_{\left(\lambda_{1}, n_{1}\right) \in I}\left|F_{2}\left(\left(\lambda_{1}, n_{1}\right), x\right)\right| \\
& \left.=\left\|F_{1}\right\|_{\infty} \sum_{\left(\lambda_{1}, n_{1}\right) \in I}\left|f_{2}\left(\lambda_{1}\right)\right| \quad \text { (where } \Xi^{*}\left(f_{2}\right)=F_{2}\right) \\
& =\left\|F_{1}\right\|_{\infty}\left\|F_{2}\right\|_{1, \ell .} .
\end{aligned}
$$

It follows that $F_{1} * F_{2} \in L^{\infty}(I \times X)^{\mathcal{S}}$. Furthermore, we have

$\left(F_{2}^{\sharp} * F_{1}^{\sharp}\right)(i, x)=\sum_{j \in I} F_{2}^{\sharp}\left(\sigma\left(\psi_{j}(x), x\right)(i), \psi_{j}(x)\right) F_{1}^{\sharp}(j, x)=$

$\sum_{j \in I} \overline{F_{2}\left(\sigma\left(\psi_{\sigma\left(\psi_{j}(x), x\right)(i)}\left(\psi_{j}(x)\right), \psi_{j}(x)\right)(0), \psi_{\sigma\left(\psi_{j}(x), x\right)(i)}\left(\psi_{j}(x)\right)\right) F_{1}\left(\sigma\left(\psi_{j}(x), x\right)(0), \psi_{j}(x)\right)}$.

Since

$$
\begin{aligned}
& \sigma\left(\psi_{\sigma\left(\psi_{j}(x), x\right)(i)}\left(\psi_{j}(x)\right), \psi_{j}(x)\right)(0) \\
& =\sigma\left(\psi_{\sigma\left(\psi_{j}(x), x\right)(i)}\left(\psi_{j}(x)\right), \psi_{i}(x)\right)\left(\sigma\left(\psi_{i}(x), \psi_{j}(x)\right)(0)\right), \\
& \left(\psi_{\sigma\left(\psi_{j}(x), x\right)(i)}\left(\psi_{j}(x)\right), \psi_{i}(x)\right) \in \mathcal{S}_{0}
\end{aligned}
$$


we get

$$
\left(F_{2}^{\sharp} * F_{1}^{\sharp}\right)(i, x)=\sum_{j \in I} \overline{F_{2}\left(\sigma\left(\psi_{i}(x), \psi_{j}(x)\right)(0), \psi_{i}(x)\right) F_{1}\left(\sigma\left(\psi_{j}(x), x\right)(0), \psi_{j}(x)\right)} .
$$

If $k=\sigma\left(\psi_{i}(x), \psi_{j}(x)\right)(0)$, then $\left(\psi_{k}\left(\psi_{i}(x)\right), \psi_{j}(x)\right) \in \mathcal{S}_{0}$, so that

$$
\begin{aligned}
F_{1}\left(\sigma\left(\psi_{j}(x), x\right)(0), \psi_{j}(x)\right) & =F_{1}\left(\sigma\left(\psi_{j}(x), \psi_{k}\left(\psi_{i}(x)\right)\left(\sigma\left(\psi_{k}\left(\psi_{i}(x)\right), x\right)(0)\right), \psi_{j}(x)\right)\right. \\
& =F_{1}\left(\sigma\left(\psi_{k}\left(\psi_{i}(x)\right), x\right)(0), \psi_{k}\left(\psi_{i}(x)\right)\right) .
\end{aligned}
$$

Together with the fact that $\left\{\sigma\left(\psi_{i}(x), \psi_{j}(x)\right)(0): j \in I\right\}=I$, it follows that

$$
\begin{aligned}
\sum_{j \in I} & \overline{F_{2}\left(\sigma\left(\psi_{i}(x), \psi_{j}(x)\right)(0), \psi_{i}(x)\right) F_{1}\left(\sigma\left(\psi_{j}(x), x\right)(0), \psi_{j}(x)\right)} \\
& =\sum_{k \in I} \overline{F_{2}\left(k, \psi_{i}(x)\right) F_{1}\left(\sigma\left(\psi_{k}\left(\psi_{i}(x)\right), x\right)(0), \psi_{k}\left(\psi_{i}(x)\right)\right)} \\
& =\sum_{k \in I} \overline{F_{1}\left(\sigma\left(\psi_{k}\left(\psi_{i}(x)\right), \psi_{i}(x)\right)\left(\sigma\left(\psi_{i}(x), x\right)(0)\right), \psi_{k}\left(\psi_{i}(x)\right)\right) F_{2}\left(k, \psi_{i}(x)\right)} \\
& =\overline{\left(F_{1} * F_{2}\right)\left(\sigma\left(\psi_{i}(x), x\right)(0), \psi_{i}(x)\right)} \\
& =\left(F_{1} * F_{2}\right)^{\sharp}(i, x) .
\end{aligned}
$$

Hence we have shown that $\left(F_{1} * F_{2}\right)^{\sharp}=F_{2}^{\sharp} * F_{1}^{\sharp}$. In fact, $F_{1} * F_{2}$ lies in $\mathcal{I}(\mathcal{R}, \mathcal{S})$. To prove this, we compute

$$
\begin{aligned}
\Omega\left(\left|F_{1} * F_{2}\right|\right) & =\int_{X} \sum_{i \in I}\left|\left(F_{1} * F_{2}\right)(i, x)\right| d \mu(x) \\
& \leq \int_{X} \sum_{i \in I} \sum_{j \in I}\left|F_{1}\left(\sigma\left(\psi_{j}(x), x\right)(i), \psi_{j}(x)\right)\right|\left|F_{2}(j, x)\right| d \mu(x) \\
& =\int_{X} \sum_{j \in I}\left(\sum_{i \in I}\left|F_{1}\left(\sigma\left(\psi_{j}(x), x\right)(i), \psi_{j}(x)\right)\right|\right)\left|F_{2}(j, x)\right| d \mu(x) \\
& =\left\|F_{1}\right\|_{1, \ell}\left\|F_{2}\right\|_{1, \ell}<\infty .
\end{aligned}
$$

From this, we have

$$
\Omega\left(\left|\left(F_{1} * F_{2}\right)^{\sharp}\right|\right)=\Omega\left(\left|F_{2}^{\sharp} * F_{1}^{\sharp}\right|\right) \leq\left\|F_{1}\right\|_{1, r}\left\|F_{2}\right\|_{1, r}<\infty .
$$

Thus $F_{1} * F_{2}$ belongs to $\mathcal{I}(\mathcal{R}, \mathcal{S})$.

Definition 9.10. Let $F_{1}$ and $F_{2}$ be in $\mathcal{I}$. We call $F_{1} * F_{2}$ the convolution of $F_{1}$ and $F_{2}$. 
Next we check that the convolution is associative. So take arbitrary $F_{1}, F_{2}$, $F_{3}$ in $\mathcal{I}(\mathcal{R}, \mathcal{S})$. As before, we choose a $\mu$-null subset $N_{0}$ of $X$ such that

$$
\begin{aligned}
& F_{k}(\sigma(y, x)(i), y)=F_{k}(i, x) \\
& \qquad \text { for all }(x, y) \in \mathcal{S}_{0}:=\mathcal{S} \cap\left(N_{0}^{c} \times N_{0}^{c}\right), i \in I \text { and } k=1,2,3 .
\end{aligned}
$$

Put $N_{1}:=\bigcup_{i, j \in I} \psi_{j}^{-1}\left(\psi_{i}^{-1}\left(N_{0}\right)\right)$ and $\mathcal{S}_{1}:=\mathcal{S} \cap\left(N_{1}^{c} \times N_{1}^{c}\right)$. Then, for any $x \in N_{1}^{c}$ and $i \in I$, we have

$$
\begin{aligned}
\left(\left(F_{1} * F_{2}\right)\right. & \left.* F_{3}\right)(i, x)=\sum_{k \in I}\left(F_{1} * F_{2}\right)\left(\sigma\left(\psi_{k}(x), x\right)(i), \psi_{k}(x)\right) F_{3}(k, x) \\
= & \sum_{k \in I} \sum_{j \in I} F_{1}\left(\sigma\left(\psi_{j}\left(\psi_{k}(x)\right), x\right)(i), \psi_{j}\left(\psi_{k}(x)\right)\right) F_{2}\left(j, \psi_{k}(x)\right) F_{3}(k, x) .
\end{aligned}
$$

Since

$$
\begin{gathered}
\sigma\left(\psi_{j}\left(\psi_{k}(x)\right), x\right)(i)=\sigma\left(\psi_{j}\left(\psi_{k}(x)\right), \psi_{\sigma\left(x, \psi_{k}(x)\right)(j)}(x)\right)\left(\sigma\left(\psi_{\sigma\left(x, \psi_{k}(x)\right)(j)}(x), x\right)(i)\right), \\
\left(\psi_{j}\left(\psi_{k}(x)\right), \psi_{\sigma\left(x, \psi_{k}(x)\right)(j)}(x)\right) \in \mathcal{S}_{0}
\end{gathered}
$$

one has

$$
\begin{aligned}
F_{1}\left(\sigma\left(\psi_{j}\left(\psi_{k}(x)\right), x\right)(i), \psi_{j}\right. & \left.\left(\psi_{k}(x)\right)\right) \\
& =F_{1}\left(\sigma\left(\psi_{\sigma\left(x, \psi_{k}(x)\right)(j)}(x), x\right)(i), \psi_{\sigma\left(x, \psi_{k}(x)\right)(j)}(x)\right) .
\end{aligned}
$$

So

$$
\begin{aligned}
& \left(\left(F_{1} * F_{2}\right) * F_{3}\right)(i, x) \\
& \quad=\sum_{k \in I} \sum_{j \in I} F_{1}\left(\sigma\left(\psi_{\sigma\left(x, \psi_{k}(x)\right)(j)}(x), x\right)(i), \psi_{\sigma\left(x, \psi_{k}(x)\right)(j)}(x)\right) F_{2}\left(j, \psi_{k}(x)\right) F_{3}(k, x) .
\end{aligned}
$$

Because $\left\{\sigma\left(x, \psi_{k}(x)\right)(j): j \in I\right\}=I$, we continue the above computation as follows:

$$
\begin{aligned}
\left(\left(F_{1} * F_{2}\right) *\right. & \left.F_{3}\right)(i, x) \\
& =\sum_{k \in I} \sum_{\ell \in I} F_{1}\left(\sigma\left(\psi_{\ell}(x), x\right)(i), \psi_{\ell}(x)\right) F_{2}\left(\sigma\left(\psi_{k}(x), x\right)(\ell), \psi_{k}(x)\right) F_{3}(k, x) \\
& =\sum_{\ell \in I} F_{1}\left(\sigma\left(\psi_{\ell}(x), x\right)(i), \psi_{\ell}(x)\right)\left(\sum_{k \in I} F_{2}\left(\sigma\left(\psi_{k}(x), x\right)(\ell), \psi_{k}(x)\right) F_{3}(k, x)\right) \\
& =\sum_{\ell \in I} F_{1}\left(\sigma\left(\psi_{\ell}(x), x\right)(i), \psi_{\ell}(x)\right)\left(F_{2} * F_{3}\right)(\ell, x)=\left(F_{1} *\left(F_{2} * F_{3}\right)\right)(i, x) .
\end{aligned}
$$

Thus the convolution is associative. 
Finally, the function $F^{0}$, i.e., $F^{0}(i, x)=\delta_{0, i}$, is the identity of $\mathcal{I}(\mathcal{R}, \mathcal{S})$. Indeed, for any $F \in \mathcal{I}(\mathcal{R}, \mathcal{S})$, we have

$$
\begin{aligned}
\left(F^{0} * F\right)(i, x) & =\sum_{j \in I} F^{0}\left(\sigma\left(\psi_{j}(x), x\right)(i), \psi_{j}(x)\right) F(j, x) \\
& =\sum_{j \in I} \delta_{0, \sigma\left(\psi_{j}(x), x\right)(i)} F(j, x)=\sum_{j \in I} \delta_{i, j} F(j, x)=F(i, x) .
\end{aligned}
$$

One can also easily show that $F * F^{0}=F$.

Therefore, we have proven that

Theorem 9.11. $\mathcal{I}(\mathcal{R}, \mathcal{S})$ is a unital involutive algebra over $\mathbb{C}$ with product $*$ and involution $\sharp$.

Let $\lambda_{1}, \lambda_{2} \in \Lambda$. Then

$$
\left(F^{\lambda_{1}} * F^{\lambda_{2}}\right)((\lambda, n), x)=\sum_{\left(\lambda^{\prime}, n^{\prime}\right) \in I} F^{\lambda_{1}}\left(\sigma\left(\psi_{\lambda^{\prime}, n^{\prime}}(x), x\right)(\lambda, n), \psi_{\lambda^{\prime}, n^{\prime}}(x)\right) F^{\lambda_{2}}\left(\left(\lambda^{\prime}, n^{\prime}\right), x\right) .
$$

As usual, take a $\mu$-null subset $N_{0}$ of $X$ such that

$$
\chi_{\mathcal{C}_{\lambda_{1}}}(z, x)=\chi_{\mathcal{C}_{\lambda_{1}}}(z, y) \text { for all }(x, y) \in \mathcal{S}_{0}:=\mathcal{S} \cap\left(N_{0}^{c} \times N_{0}^{c}\right) \text { and all } z \in \mathcal{R}(x) \text {. }
$$

Put $N_{1}:=\bigcup_{i, j \in I} \psi_{j}^{-1}\left(\psi_{i}^{-1}\left(N_{0}\right)\right)$. By (the proof of) [2, Theorem 3.8], there exists a $\mu$-null subset $N_{2}$ of $X$ such that, for any $x \in N_{2}^{c}$ and $\left(\lambda_{2}, k\right),\left(\psi_{\lambda_{2}, k}(x), \psi_{\lambda, n}(x)\right) \in$ $\mathcal{C}_{\lambda_{1}}$ if and only if $\psi_{\lambda, n}(x) \in \bigsqcup_{\ell=1}^{n_{\lambda_{1}}} \mathcal{S}\left(\psi_{\lambda_{1}, \ell}\left(\psi_{\lambda_{2}, k}(x)\right)\right.$. Now set $N_{3}:=N_{1} \cup N_{2}$ and $\mathcal{S}_{1}:=\mathcal{S} \cap\left(N_{3}^{c} \times N_{3}^{c}\right)$. Then, for any $x \in N_{3}^{c}$ and $i \in I$, we have

$$
\begin{aligned}
F^{\lambda_{1}} & \left(\sigma\left(\psi_{\lambda^{\prime}, n^{\prime}}(x), x\right)(\lambda, n), \psi_{\lambda^{\prime}, n^{\prime}}(x)\right) \\
& =\chi_{\mathcal{C}_{\lambda_{1}}}\left(\psi_{\lambda^{\prime}, n^{\prime}}(x), \psi_{\sigma\left(\psi_{\lambda^{\prime}, n^{\prime}}(x), x\right)(\lambda, n)}\left(\psi_{\lambda^{\prime}, n^{\prime}}(x)\right)\right) \\
& =\chi_{\mathcal{C}_{\lambda_{1}}}\left(\psi_{\lambda^{\prime}, n^{\prime}}(x), \psi_{\lambda, n}(x)\right) \quad\left(\operatorname{as}\left(\psi_{\sigma\left(\psi_{\lambda^{\prime}, n^{\prime}}(x), x\right)(\lambda, n)}\left(\psi_{\lambda^{\prime}, n^{\prime}}(x)\right), \psi_{\lambda, n}(x)\right) \in \mathcal{S}_{0}\right)
\end{aligned}
$$

Thus

$$
\begin{aligned}
\left(F^{\lambda_{1}} * F^{\lambda_{2}}\right)((\lambda, n), x) & =\sum_{\left(\lambda^{\prime}, n^{\prime}\right) \in I} \chi_{\mathcal{C}_{\lambda_{1}}}\left(\psi_{\lambda^{\prime}, n^{\prime}}(x), \psi_{\lambda, n}(x)\right) F^{\lambda_{2}}\left(\left(\lambda^{\prime}, n^{\prime}\right), x\right) \\
& =\sum_{k=1}^{n_{\lambda_{2}}} \chi_{\mathcal{C}_{\lambda_{1}}}\left(\psi_{\lambda_{2}, k}(x), \psi_{\lambda, n}(x)\right) \quad\left(\text { as } \Xi^{*}\left(\delta_{\lambda_{2}}\right)=F^{\lambda_{2}}\right) .
\end{aligned}
$$

We will show that $F^{\lambda_{1}} * F^{\lambda_{2}} \in \mathcal{I}_{0}(\mathcal{R}, \mathcal{S})$. For this, we define a subset $K_{\lambda_{2}, \lambda}^{\lambda_{1}}$ of $X$ by

$$
K_{\lambda_{2}, \lambda}^{\lambda_{1}}=\bigcup_{k=1}^{n_{\lambda}} \bigcup_{k_{2}=1}^{n_{\lambda_{2}}}\left(\left(\psi_{\lambda_{2}, k_{2}} \times \psi_{\lambda, k}\right) \circ g_{0}\right)^{-1}\left(\mathcal{C}_{\lambda_{1}}\right),
$$

where $g_{0}: X \rightarrow X \times X$ is defined by $g_{0}(x):=(x, x)$. We claim that $K_{\lambda_{2}, \lambda}^{\lambda_{1}}$ is 
measurable. Indeed, since $\left\{\psi_{i}\right\}_{i \in I}$ are choice functions, $\psi_{i}$ is a measurable map for each $i \in I$. So, for each $i, j \in I$,

$$
\left(\left(\psi_{i} \times \psi_{j}\right) \circ g_{0}\right)^{-1}\left(\mathcal{C}_{\lambda}\right)
$$

is a measurable subset of $X$. Hence our claim is proven.

We claim that $K_{\lambda_{2}, \lambda}^{\lambda_{1}}$ is an $\mathcal{S}$-invariant set up to a null set. Indeed, by [2, Section 3, Remark (1)], there exists a $\mu$-null subset $N_{\lambda_{2}, \lambda}$ of $X$ such that, for each $(x, y) \in \mathcal{S} \cap\left(N_{\lambda_{2}, \lambda}^{c} \times N_{\lambda_{2}, \lambda}^{c}\right), k_{2} \in\left\{1, \ldots, n_{\lambda_{2}}\right\}, k \in\left\{1, \ldots, n_{\lambda}\right\}$, there exist $m_{2} \in\left\{1, \ldots, n_{\lambda_{2}}\right\}$ and $m \in\left\{1, \ldots, n_{\lambda}\right\}$ which satisfy $\left(\psi_{\lambda_{2}, m_{2}}(y), \psi_{\lambda_{2}, k_{2}}(x)\right) \in \mathcal{S}$ and $\left(\psi_{\lambda, m}(y), \psi_{\lambda, k}(x)\right) \in \mathcal{S}$. In particular, if $\left(\psi_{\lambda_{2}, k_{2}}(x), \psi_{\lambda, k}(x)\right) \in \mathcal{C}_{\lambda_{1}}$, then

$$
\begin{aligned}
& \left(\psi_{\lambda_{2}, m_{2}}(y), \psi_{\lambda, m}(y)\right) \\
& \quad=\left(\psi_{\lambda_{2}, m_{2}}(y), \psi_{\lambda_{2}, k_{2}}(x)\right)\left(\psi_{\lambda_{2}, k_{2}}(x), \psi_{\lambda, k}(x)\right)\left(\psi_{\lambda, k}(x), \psi_{\lambda, m}(y)\right) \in \mathcal{C}_{\lambda_{1}}
\end{aligned}
$$

This means that if $x \in K_{\lambda_{2}, \lambda}^{\lambda_{1}} \cap N_{\lambda_{2}, \lambda}^{c}$, then $\mathcal{S}(x) \cap N_{\lambda_{2}, \lambda}^{c} \subseteq K_{\lambda_{2}, \lambda}^{\lambda_{1}}$. So our claim is proven. Since $\mathcal{S}$ is ergodic, we conclude that $K_{\lambda_{2}, \lambda}^{\lambda_{1}}$ is either null or conull in $X$ for each $\lambda \in \Lambda$.

We next claim that there exist a finite subset $\Lambda_{0}$ of $\Lambda$ and a measurable nonnull subset $E$ of $X$ such that $K_{\lambda_{2}, \lambda}^{\lambda_{1}}$ is contained in $E^{c}$ for all $\lambda \in\left(\Lambda \backslash \Lambda_{0}\right)$. Indeed, put $E_{\lambda_{0}}^{k_{1}, k_{2}}:=\left\{x \in X:\left(x, \psi_{\lambda_{1}, k_{1}}\left(\psi_{\lambda_{2}, k_{2}}(x)\right)\right) \in \mathcal{C}_{\lambda_{0}}\right\}$ for each $\lambda_{0} \in \Lambda, k_{1} \in\left\{1, \ldots, n_{\lambda_{1}}\right\}$ and $k_{2} \in\left\{1, \ldots, n_{\lambda_{2}}\right\}$. Since $\mathcal{C}_{\lambda_{0}}$ is measurable and $x \mapsto\left(x, \psi_{\lambda_{1}, k_{1}}\left(\psi_{\lambda_{2}, k_{2}}(x)\right)\right)$ is a measurable map from $X$ to $\mathcal{R}$ up to a null set, $E_{\lambda_{0}}^{k_{1}, k_{2}}$ is a measurable subset of $X$. Since $\left\{\mathcal{C}_{\lambda_{0}}\right\}_{\lambda_{0} \in \Lambda}$ is a countable measurable partition of $\mathcal{R}$ up to a null set, $\left\{E_{\lambda_{0}}^{k_{1}, k_{2}}\right\}_{\lambda_{0} \in \Lambda}$ is a measurable partition of $X$ up to a null set. In particular, for any $\left(k_{1}, k_{2}\right)$ and each measurable nonnull subset $E$ of $X$, there exists $\lambda_{0} \in \Lambda$ such that $E \cap E_{\lambda_{0}}^{k_{1}, k_{2}}$ is a nonnull measurable subset of $X$. By using induction, there exists $\left\{\lambda_{k_{1}, k_{2}}\right\}_{k_{1}, k_{2}} \subseteq \Lambda$ such that $\bigcap_{k_{1}=1}^{n_{\lambda_{1}}} \bigcap_{k_{2}=1}^{n_{\lambda_{2}}} E_{\lambda_{k_{1}, k_{2}}}^{k_{1}, k_{2}}$ is a nonnull measurable subset of $X$. Put $E:=\bigcap_{k_{1}=1}^{n_{\lambda_{1}}} \bigcap_{k_{2}=1}^{n_{\lambda_{2}}} E_{\lambda_{k_{1}, k_{2}}}^{k_{1}, k_{2}}$ and $\Lambda_{0}:=\left\{\lambda_{k_{1}, k_{2}}: k_{1} \in\left\{1, \ldots, n_{\lambda_{1}}\right\}, k_{2} \in\right.$ $\left.\left\{1, \ldots, n_{\lambda_{2}}\right\}\right\}$. By construction, $\left|\Lambda_{0}\right| \leq n_{\lambda_{1}} n_{\lambda_{2}}$. In particular, $\Lambda_{0}$ is a finite subset of $\Lambda$. Moreover, since $E \subseteq E_{\lambda_{k_{1}, k_{2}}}^{k_{1}, k_{2}}$ for each $k_{1} \in\left\{1, \ldots, n_{\lambda_{1}}\right\}, k_{2} \in\left\{1, \ldots, n_{\lambda_{2}}\right\}$, $\left(x, \psi_{\lambda_{1}, k_{1}}\left(\psi_{\lambda_{2}, k_{2}}(x)\right)\right)$ belongs to $\mathcal{C}_{\lambda_{k_{1}, k_{2}}}$ for each $x \in E$ and $k_{1} \in\left\{1, \ldots, n_{\lambda_{1}}\right\}, k_{2} \in$ $\left\{1, \ldots, n_{\lambda_{2}}\right\}$. By (8.1), together with the equivalent conditions displayed in the proof of Lemma 8.5 we have $\mathcal{S}\left(\psi_{\lambda_{1}, k_{1}}\left(\psi_{\lambda_{2}, k_{2}}(x)\right)\right) \subseteq \bigcup_{k=1}^{n_{\lambda_{k_{1}}, k_{2}}} \mathcal{S}\left(\psi_{\lambda_{k_{1}, k_{2}}, k}(x)\right)$ for each $x \in E$ and $k_{1} \in\left\{1, \ldots, n_{\lambda_{1}}\right\}, k_{2} \in\left\{1, \ldots, n_{\lambda_{2}}\right\}$. Since $\left\{\mathcal{S}\left(\psi_{i}(x)\right)\right\}_{i \in I}$ are mutually disjoint, it follows that if $\lambda \notin \Lambda_{0}$, then for each $k \in\left\{1, \ldots, n_{\lambda}\right\}$, we have

$$
\psi_{\lambda, k}(x) \in\left(\bigcup_{k_{1}=1}^{n_{\lambda_{1}}} \bigcup_{k_{2}=1}^{n_{\lambda_{2}}} \bigcup_{k=1}^{n_{\lambda_{k_{1}}, k_{2}}} \mathcal{S}\left(\psi_{\lambda_{k_{1}, k_{2}}, k}(x)\right)\right)^{c} \subseteq\left(\bigcup_{k_{1}=1}^{n_{\lambda_{1}}} \bigcup_{k_{2}=1}^{n_{\lambda_{2}}} \mathcal{S}\left(\psi_{\lambda_{1}, k_{1}}\left(\psi_{\lambda_{2}, k_{2}}(x)\right)\right)^{c},\right.
$$

and $K_{\lambda_{1}, \lambda}^{\lambda_{1}} \subseteq E^{c}$. So our claim is proven. 
By using the above results, we conclude that $K_{\lambda_{2}, \lambda}^{\lambda_{1}}$ is null if $\lambda \in \Lambda \backslash \Lambda_{0}$. We claim that the support of $F^{\lambda_{1}} * F^{\lambda_{2}}$ is contained in $\left\{(\lambda, k): \lambda \in \Lambda_{0}, k \in\right.$ $\left.\left\{1, \ldots, n_{\lambda}\right\}\right\} \times X$ up to a null set. Indeed, put $N:=\bigcup_{\lambda^{\prime} \in \Lambda \backslash \Lambda_{0}} K_{\lambda_{2}, \lambda^{\prime}}^{\lambda_{1}}$. Since $\Lambda \backslash \Lambda_{0}$ is a countable set and $K_{\lambda_{2}, \lambda}^{\lambda_{1}}$ is a $\mu$-null set for each $\lambda \in \Lambda \backslash \Lambda_{0}, N$ is a $\mu$-null subset of $X$. In particular, $I \times N$ is null in $I \times X$. On the other hand, suppose that $((\lambda, n), x) \in\left\{(\lambda, k): \lambda \in \Lambda \backslash \Lambda_{0}, k \in\left\{1, \ldots, n_{\lambda}\right\}\right\} \times N^{c}$. Since $\lambda \in \Lambda \backslash \Lambda_{0}$, $x$ belongs to $N^{c}=\left(\bigcup_{\lambda \in \Lambda \backslash \Lambda_{0}} K_{\lambda_{2}, \lambda}^{\lambda_{1}}\right)^{c} \subseteq\left(K_{\lambda_{2}, \lambda}^{\lambda_{1}}\right)^{c}$. By the definition of $K_{\lambda_{2}, \lambda}^{\lambda_{1}}$, we have $\left(\psi_{\lambda_{1}, k}(x), \psi_{\lambda, k}(x)\right) \notin \mathcal{C}_{\lambda_{2}}$ for each $k \in\left\{1, \ldots, n_{\lambda_{2}}\right\}$. It follows that

$$
\left(F^{\lambda_{1}} * F^{\lambda_{2}}\right)((\lambda, n), x)=\sum_{k=1}^{n_{\lambda_{2}}} \chi_{\mathcal{C}_{\lambda_{1}}}\left(\psi_{\lambda_{2}, k}(x), \psi_{\lambda, n}(x)\right)=0 .
$$

So the support of $F^{\lambda_{1}} * F^{\lambda_{2}}$ is contained in

$$
\begin{aligned}
\{(I \backslash\{(\lambda, k): \lambda \in & \left.\left.\left.\Lambda \backslash \Lambda_{0}, k \in\left\{1, \ldots, n_{\lambda}\right\}\right\}\right) \times N^{c}\right\} \cup\left(I \times\left(N^{c}\right)^{c}\right) \\
& \left.=\left\{\left\{(\lambda, k): \lambda \in \Lambda_{0}, k \in\left\{1, \ldots, n_{\lambda}\right\}\right\}\right) \times N^{c}\right\} \cup(I \times N)
\end{aligned}
$$

and our claim is proven. Therefore, there exists $\left\{c_{\lambda}\right\}_{\lambda \in \Lambda_{0}}$ such that $F^{\lambda_{1}} * F^{\lambda_{2}}=$ $\sum_{\lambda \in \Lambda_{0}} c_{\lambda} F^{\lambda}$. Since $\Lambda_{0}$ is finite, we conclude that $F^{\lambda_{1}} * F^{\lambda_{2}} \in \mathcal{I}_{0}(\mathcal{R}, \mathcal{S})$. Thus we have shown

Proposition 9.12. $\mathcal{I}_{0}(\mathcal{R}, \mathcal{S})$ is a unital involutive subalgebra of $\mathcal{I}(\mathcal{R}, \mathcal{S})$.

Definition 9.13. We call $\mathcal{I}_{0}(\mathcal{R}, \mathcal{S})$ the algebraic Hecke algebra associated with the Hecke pair $(\mathcal{R}, \mathcal{S})$.

\section{§9.3. Hecke von Neumann algebras}

Our next objective is to represent the involutive algebra $\mathcal{I}(\mathcal{R}, \mathcal{S})$ on the GNS Hilbert space $H_{\Omega}$. For this, we begin with the following lemma.

Lemma 9.14. Let $F$ be in $\mathcal{I}(\mathcal{R}, \mathcal{S})$. Then, for a.e. $x \in X$ and all $i \in I$, we have

$$
\sum_{j \in I} F\left(\sigma\left(\psi_{j}(x), x\right)(i), \psi_{j}(x)\right)=\sum_{\lambda \in \Lambda} f\left(\lambda^{-1}\right) n_{\lambda}
$$

where $\Xi^{*}(f)=F$.

Proof. There exists a $\mu$-null subset $N_{0}$ of $X$ such that

$$
F^{\sharp}(\sigma(y, x)(i), y)=F^{\sharp}(i, x) \text { for all }(x, y) \in \mathcal{S}_{0}:=\mathcal{S} \cap\left(N_{0}^{c} \times N_{0}^{c}\right) \text { and all } i \in I .
$$

Put $N_{1}:=\bigcup_{i, j \in I} \psi_{j}^{-1}\left(\psi_{i}^{-1}\left(N_{0}\right)\right)$ and $\mathcal{S}_{1}:=\mathcal{S} \cap\left(N_{1}^{c} \times N_{1}^{c}\right)$. Then, for any $x \in N_{1}^{c}$ and $i \in I$, we have 


$$
\begin{aligned}
\sum_{j \in I} F( & \left.\sigma\left(\psi_{j}(x), x\right)(i), \psi_{j}(x)\right)=\sum_{j \in I}\left(F^{\sharp}\right)^{\sharp}\left(\sigma\left(\psi_{j}(x), x\right)(i), \psi_{j}(x)\right) \\
= & \sum_{j \in I} \overline{F^{\sharp}\left(\sigma\left(\psi_{\sigma\left(\psi_{j}(x), x\right)(i)}\left(\psi_{j}(x)\right), \psi_{j}(x)\right)(0), \psi_{\sigma\left(\psi_{j}(x), x\right)(i)}\left(\psi_{j}(x)\right)\right)} \\
= & \sum_{j \in I} \overline{F^{\sharp}\left(\sigma\left(\psi_{i}(x), \psi_{j}(x)\right)(0), \psi_{i}(x)\right)} \quad\left(\text { as }\left(\psi_{\sigma\left(\psi_{j}(x), x\right)(i)}\left(\psi_{j}(x)\right), \psi_{i}(x)\right) \in \mathcal{S}_{0}\right) \\
= & \sum_{j \in I} \overline{F^{\sharp}\left(\sigma\left(\psi_{i}(x), x\right)(j), \psi_{i}(x)\right)} \\
= & \sum_{j \in I} \overline{F^{\sharp}\left(j, \psi_{i}(x)\right)} \quad\left(\text { as }\left\{\sigma\left(\psi_{i}(x), x\right)(j): j \in I\right\}=I\right) \\
= & \sum_{(\lambda, n) \in I} \overline{F^{\sharp}\left((\lambda, n), \psi_{i}(x)\right)} .
\end{aligned}
$$

By the proof of Lemma 9.9 we see that $F^{\sharp}\left((\lambda, n), \psi_{i}(x)\right)=\overline{f\left(\lambda^{-1}\right)}$ when $\Xi^{*}(f)=F$. Therefore, we obtain our assertion.

In the preceding subsection we defined the convolution only for the elements in $\mathcal{I}(\mathcal{R}, \mathcal{S})$. We now extend it to the convolution of an element in $\mathcal{I}(\mathcal{R}, \mathcal{S})$ and an element in $\mathfrak{n}_{\Omega}$ in the obvious way.

Lemma 9.15. Let $F$ be in $\mathcal{I}(\mathcal{R}, \mathcal{S})$ and $F_{1}$ be in $\mathfrak{n}_{\Omega}$. Then both $F * F_{1}$ and $F_{1} * F$ belong to $\mathfrak{n}_{\Omega}$ and satisfy

$$
\left\|F * F_{1}\right\|_{2} \leq\left(\|F\|_{1, \ell}\|F\|_{1, r}\right)^{1 / 2}\left\|F_{1}\right\|_{2}, \quad\left\|F_{1} * F\right\|_{2} \leq\|F\|_{1, \ell}\left\|F_{1}\right\|_{2} .
$$

Proof. By Lemma 9.14, for a.e. $x \in X$ and all $i \in I$,

$$
\begin{aligned}
\left|\left(F * F_{1}\right)(i, x)\right| & \leq \sum_{j \in I}\left|F\left(\sigma\left(\psi_{j}(x), x\right)(i), \psi_{j}(x)\right)\right|\left|F_{1}(j, x)\right| \\
& \leq\left\|F_{1}\right\|_{\infty} \sum_{j \in I}\left|F\left(\sigma\left(\psi_{j}(x), x\right)(i), \psi_{j}(x)\right)\right|=\|F\|_{1, r}\left\|F_{1}\right\|_{\infty} .
\end{aligned}
$$

Moreover, for a.e. $x \in X$ and any $i \in I$, we get

$$
\begin{aligned}
\left|\left(F_{1} * F\right)(i, x)\right| & \leq \sum_{j \in I}\left|F_{1}\left(\sigma\left(\psi_{j}(x), x\right)(i), \psi_{j}(x)\right)\right||F(j, x)| \\
& \leq\left\|F_{1}\right\|_{\infty} \sum_{j \in I}|F(j, x)|=\|F\|_{1, \ell}\left\|F_{1}\right\|_{\infty} .
\end{aligned}
$$

Thus $F * F_{1}$ and $F_{1} * F$ belong to $L^{\infty}(I \times X)$. Both are $\mathcal{S}$-invariant, as can be checked as in the previous subsection. 
Next we show that $F * F_{1}$ lies in $\mathfrak{n}_{\Omega}$. By the Cauchy-Schwarz inequality and Lemma 9.14 , we obtain

$$
\begin{aligned}
\int_{X} \sum_{i \in I} \mid & \left.\left(F * F_{1}\right)(i, x)\right|^{2} d \mu(x) \\
= & \int_{X} \sum_{i \in I}\left(\sum_{j \in I}\left|F\left(\sigma\left(\psi_{j}(x), x\right)(i), \psi_{j}(x)\right)\right|\left|F_{1}(j, x)\right|\right)^{2} d \mu(x) \\
\leq & \int_{X} \sum_{i \in I}\left(\sum_{j \in I}\left|F\left(\sigma\left(\psi_{j}(x), x\right)(i), \psi_{j}(x)\right)\right|\right) \\
& \times\left(\sum_{j \in I}\left|F\left(\sigma\left(\psi_{j}(x), x\right)(i), \psi_{j}(x)\right)\right|\left|F_{1}(j, x)\right|^{2}\right) d \mu(x) \\
= & \|F\|_{1, r} \int_{X} \sum_{i \in I}\left(\sum_{j \in I}\left|F\left(\sigma\left(\psi_{j}(x), x\right)(i), \psi_{j}(x)\right)\right|\left|F_{1}(j, x)\right|^{2}\right) d \mu(x) \\
= & \|F\|_{1, r} \int_{X} \sum_{j \in I}\left(\sum_{i \in I}\left|F\left(\sigma\left(\psi_{j}(x), x\right)(i), \psi_{j}(x)\right)\right|\right)\left|F_{1}(j, x)\right|^{2} d \mu(x) \\
= & \|F\|_{1, \ell}\|F\|_{1, r}\left\|F_{1}\right\|_{2}^{2}<\infty .
\end{aligned}
$$

Thus $F * F_{1} \in \mathfrak{n}_{\Omega}$ and $\left\|F * F_{1}\right\|_{2} \leq\left(\|F\|_{1, \ell}\|F\|_{1, r}\right)^{1 / 2}\left\|F_{1}\right\|_{2}$. We also have

$$
\begin{aligned}
\int_{X} \sum_{i \in I}\left|\left(F_{1} * F\right)(i, x)\right|^{2} d \mu(x) \\
\quad=\int_{X} \sum_{i \in I}\left(\sum_{j \in I}\left|F_{1}\left(\sigma\left(\psi_{j}(x), x\right)(i), \psi_{j}(x)\right)\right||F(j, x)|\right)^{2} \mu(x) \\
\leq \int_{X} \sum_{i \in I}\left(\sum_{j \in I}\left|F_{1}\left(\sigma\left(\psi_{j}(x), x\right)(i), \psi_{j}(x)\right)\right|^{2}|F(j, x)|\right)\left(\sum_{j \in I}|F(j, x)|\right) d \mu(x) \\
=\|F\|_{1, \ell} \int_{X} \sum_{i \in I}\left(\sum_{j \in I}\left|F_{1}\left(\sigma\left(\psi_{j}(x), x\right)(i), \psi_{j}(x)\right)\right|^{2}|F(j, x)|\right) d \mu(x) \\
=\|F\|_{1, \ell} \int_{X} \sum_{j \in I}\left(\sum_{i \in I}\left|F_{1}\left(\sigma\left(\psi_{j}(x), x\right)(i), \psi_{j}(x)\right)\right|^{2}\right)|F(j, x)| d \mu(x) \\
=\|F\|_{1, \ell}^{2}\left\|F_{1}\right\|_{2}^{2}<\infty
\end{aligned}
$$

Thus $F_{1} * F \in \mathfrak{n}_{\Omega}$ and $\left\|F_{1} * F\right\|_{2} \leq\|F\|_{1, \ell}\left\|F_{1}\right\|_{2}$.

Thanks to Lemma 9.15, the equations

$$
\begin{aligned}
& \pi_{\ell}^{0}(F) \Lambda_{\Omega}\left(F_{1}\right):=\Lambda_{\Omega}\left(F * F_{1}\right) \\
& \pi_{r}^{0}(F) \Lambda_{\Omega}\left(F_{1}\right):=\Lambda_{\Omega}\left(F_{1} * F\right)
\end{aligned} \quad\left(F \in \mathcal{I}(\mathcal{R}, \mathcal{S}), F_{1} \in \mathfrak{n}_{\Omega}\right)
$$


define bounded linear operators $\pi_{\ell}^{0}(F)$ and $\pi_{r}^{0}(F)$ on the pre-Hilbert space $\Lambda_{\Omega}\left(\mathfrak{n}_{\Omega}\right)$. So $\pi_{\ell}^{0}(F)$ and $\pi_{r}^{0}(F)$ can be uniquely extended to bounded operators $\pi_{\ell}(F)$ and $\pi_{r}(F)$ on $H_{\Omega}$.

Lemma 9.16. The map $\pi_{\ell}: \mathcal{I}(\mathcal{R}, \mathcal{S}) \rightarrow B\left(H_{\Omega}\right)$ is an involution-preserving homomorphism from $\mathcal{I}(\mathcal{R}, \mathcal{S})$ into $B\left(H_{\Omega}\right)$ satisfying $\left\|\pi_{\ell}(F)\right\| \leq\left(\|F\|_{1, \ell}\|F\|_{1, r}\right)^{1 / 2}$ for all $F \in \mathcal{I}(\mathcal{R}, \mathcal{S})$. On the other hand, the map $\pi_{r}: \mathcal{I}(\mathcal{R}, \mathcal{S}) \rightarrow B\left(H_{\Omega}\right)$ is an antihomomorphism from $\mathcal{I}(\mathcal{R}, \mathcal{S})$ into $B\left(H_{\Omega}\right)$ satisfying $\left\|\pi_{r}(F)\right\| \leq\|F\|_{1, \ell}$ for all $F \in \mathcal{I}(\mathcal{R}, \mathcal{S})$.

Proof. It is obvious that $\pi_{\ell}$ and $\pi_{r}$ are linear.

Since $\Lambda_{\Omega}(\mathcal{I}(\mathcal{R}, \mathcal{S}))$ is dense in $H_{\Omega}$ as noted in the previous subsection, $\pi_{\ell}(F)$ and $\pi_{r}(F)$ are completely characterized by the identities

$$
\pi_{\ell}(F) \Lambda_{\Omega}\left(F_{1}\right)=\Lambda_{\Omega}\left(F * F_{1}\right), \quad \pi_{r}(F) \Lambda_{\Omega}\left(F_{1}\right)=\Lambda_{\Omega}\left(F_{1} * F\right),
$$

where $F_{1} \in \mathcal{I}(\mathcal{R}, \mathcal{S})$. Hence both $\pi_{\ell}$ being multiplicative and $\pi_{r}$ being antimultiplicative follow from the associativity of the convolution.

It remains to show that $\pi_{\ell}\left(F^{\sharp}\right)=\pi_{\ell}(F)^{*}$ for all $F \in \mathcal{I}(\mathcal{R}, \mathcal{S})$. So let $F, F_{1}$, $F_{2}$ be in $\mathcal{I}(\mathcal{R}, \mathcal{S})$. Fubini's theorem yields

$$
\begin{aligned}
& \left(\pi_{\ell}\left(F^{\sharp}\right) \Lambda_{\Omega}\left(F_{1}\right) \mid \Lambda_{\Omega}\left(F_{2}\right)\right)=\left(\Lambda_{\Omega}\left(F^{\sharp} * F_{1}\right) \mid \Lambda_{\Omega}\left(F_{2}\right)\right)=\Omega\left(\overline{F_{2}}\left(F^{\sharp} * F_{1}\right)\right) \\
= & \int_{X} \sum_{i \in I}\left(F^{\sharp} * F_{1}\right)(i, x) \overline{F_{2}(i, x)} d \mu(x) \\
= & \int_{X} \sum_{i \in I}\left(\sum_{j \in I} F^{\sharp}\left(\sigma\left(\psi_{j}(x), x\right)(i), \psi_{j}(x)\right) F_{1}(j, x)\right) \overline{F_{2}(i, x)} d \mu(x) \\
= & \int_{X} \sum_{i \in I}\left(\sum_{j \in I} \overline{F\left(\sigma\left(\psi_{\sigma\left(\psi_{j}(x), x\right)(i)}\left(\psi_{j}(x)\right), \psi_{j}(x)\right)(0), \psi_{\sigma\left(\psi_{j}(x), x\right)(i)}\left(\psi_{j}(x)\right)\right)} F_{1}(j, x)\right) \\
= & \int_{X} \sum_{j \in I} F_{1}(j, x) \\
& \times\left(\sum_{i \in I} \overline{F\left(\sigma\left(\psi_{\sigma\left(\psi_{j}(x), x\right)(i)}\left(\psi_{j}(x)\right), \psi_{j}(x)\right)(0), \psi_{\sigma\left(\psi_{j}(x), x\right)(i)}\left(\psi_{j}(x)\right)\right) F_{2}(i, x)}\right) d \mu(x) .
\end{aligned}
$$

Since $\left(\psi_{\sigma\left(\psi_{j}(x), x\right)(i)}\left(\psi_{j}(x)\right), \psi_{i}(x)\right) \in \mathcal{S}$ for a.e. $x \in X$, we have

$$
\begin{aligned}
& F\left(\sigma\left(\psi_{\sigma\left(\psi_{j}(x), x\right)(i)}\left(\psi_{j}(x)\right), \psi_{j}(x)\right)(0), \psi_{\sigma\left(\psi_{j}(x), x\right)(i)}\left(\psi_{j}(x)\right)\right) \\
& =F\left(\sigma\left(\psi_{i}(x), \psi_{j}(x)\right)(0), \psi_{i}(x)\right)=F\left(\sigma\left(\psi_{i}(x), x\right)(j), \psi_{i}(x)\right)
\end{aligned}
$$


for a.e. $x \in X$. Thus

$$
\begin{aligned}
\left(\pi_{\ell}\left(F^{\sharp}\right) \Lambda_{\Omega}\left(F_{1}\right) \mid \Lambda_{\Omega}\left(F_{2}\right)\right) & \\
& =\int_{X} \sum_{j \in I} F_{1}(j, x)\left(\sum_{i \in I} \overline{F\left(\sigma\left(\psi_{i}(x), x\right)(j), \psi_{i}(x)\right) F_{2}(i, x)}\right) d \mu(x) \\
& =\int_{X} \sum_{j \in I} F_{1}(j, x) \overline{\left(F * F_{2}\right)(j, x)} d \mu(x) \\
& =\left(\Lambda_{\Omega}\left(F_{1}\right) \mid \Lambda_{\Omega}\left(F * F_{2}\right)\right)=\left(\Lambda_{\Omega}\left(F_{1}\right) \mid \pi_{\ell}(F) \Lambda_{\Omega}\left(F_{2}\right)\right) .
\end{aligned}
$$

This proves that $\pi_{\ell}\left(F^{\sharp}\right)=\pi_{\ell}(F)^{*}$.

Let $F \in \mathcal{I}(\mathcal{R}, \mathcal{S})$. We define a Borel function $\tilde{F}$ on $I \times X$ by

$$
\tilde{F}((\lambda, n), x):=\left[\frac{n_{\lambda^{-1}}}{n_{\lambda}}\right]^{1 / 2} F^{\sharp}((\lambda, n), x) \quad(((\lambda, n), x) \in I \times X) .
$$

So, if $\Xi^{*}(f)=F$, then, as in the proof of Lemma 9.9 , we get

$$
\tilde{F}((\lambda, n), x)=\left[\frac{n_{\lambda^{-1}}}{n_{\lambda}}\right]^{1 / 2} \overline{f\left(\lambda^{-1}\right)} .
$$

Since $\left(\|F\|_{1, \ell}=\right) \sum_{\lambda \in \Lambda}|f(\lambda)| n_{\lambda}<\infty$, there is a positive constant $C$ such that $|f(\lambda)| n_{\lambda} \leq C$ for all $\lambda \in \Lambda$. From this, we obtain

$$
|\tilde{F}((\lambda, n), x)| \leq\left(\left|f\left(\lambda^{-1}\right)\right| n_{\lambda^{-1}}\right)^{1 / 2}\left|f\left(\lambda^{-1}\right)\right|^{1 / 2} \leq C^{1 / 2}\|f\|_{\infty}^{1 / 2} .
$$

Hence $\tilde{F} \in L^{\infty}(I \times X)$. Since $F^{\sharp}$ is $\mathcal{S}$-invariant, one has $F^{\sharp}(\sigma(y, x)(\lambda, n), y)=$ $F^{\sharp}((\lambda, n), x)$ for a.e. $(x, y) \in \mathcal{S}$ and all $(\lambda, n) \in I$. As we showed in the proof of Lemma 9.7, we have $\sigma(y, x)(\lambda, n)=(\lambda, m)$ for some $m=1, \ldots, n_{\lambda}$ whenever $(x, y) \in \mathcal{S}$. From this, we see that $\tilde{F}$ is also $\mathcal{S}$-invariant. Thus $\tilde{F} \in L^{\infty}(I \times X)^{\mathcal{S}}$. Moreover, we get

$$
\begin{aligned}
\Omega\left(|\tilde{F}|^{2}\right) & =\sum_{\lambda \in \Lambda}\left|\left(\Xi^{*}\right)^{-1}(\tilde{F})(\lambda)\right|^{2} n_{\lambda}=\sum_{\lambda \in \Lambda}\left|\left[\frac{n_{\lambda^{-1}}}{n_{\lambda}}\right]^{1 / 2} \overline{f\left(\lambda^{-1}\right)}\right|^{2} n_{\lambda} \\
& =\sum_{\lambda \in \Lambda}\left|f\left(\lambda^{-1}\right)\right|^{2} n_{\lambda^{-1}}=\sum_{\lambda \in \Lambda}|f(\lambda)|^{2} n_{\lambda}=\left\|\Lambda_{\Omega}(F)\right\|_{2}^{2} .
\end{aligned}
$$

Consequently, $\tilde{F} \in \mathfrak{n}_{\Omega}$ and $\left\|\Lambda_{\Omega}(\tilde{F})\right\|_{2}=\left\|\Lambda_{\Omega}(F)\right\|_{2}$. It is now easy to see that the map $\Lambda_{\Omega}(F) \mapsto \Lambda_{\Omega}(\tilde{F})$ extends uniquely to a conjugate-linear isometry $\mathbf{J}$ on $H_{\Omega}$. Note that $\mathbf{J} \Lambda_{\Omega}\left(F^{\lambda}\right)=\left[n_{\lambda} / n_{\lambda^{-1}}\right]^{1 / 2} \Lambda_{\Omega}\left(F^{\lambda^{-1}}\right)$ for any $\lambda \in \Lambda$, which ensures that $\mathbf{J}$ is indeed unitary and satisfies $\mathbf{J}^{2}=1$. 
We introduce a subspace $\mathfrak{D}(\nabla)$ of $H_{\Omega}$ by

$$
\mathfrak{D}(\nabla):=\left\{\xi \in H_{\Omega}: \sum_{\lambda \in \Lambda}\left[\frac{n_{\lambda^{-1}}}{n_{\lambda}}\right]^{2}\left\|F^{\lambda} \xi\right\|_{2}^{2}<\infty\right\} .
$$

Clearly, the vector $\Lambda_{\Omega}\left(F^{\lambda}\right)$ belongs to $\mathfrak{D}(\nabla)$ for all $\lambda \in \Lambda$. So $\mathfrak{D}(\nabla)$ is dense in $H_{\Omega}$. Then define a densely defined linear operator $\nabla$ with domain $\mathfrak{D}(\nabla)$ by

$$
\nabla \xi:=\sum_{\lambda \in \Lambda} \frac{n_{\lambda^{-1}}}{n_{\lambda}} F^{\lambda} \xi \quad(\forall \xi \in \mathfrak{D}(\nabla)) .
$$

We have $\nabla \Lambda_{\Omega}\left(F^{\lambda}\right)=\left(n_{\lambda^{-1}} / n_{\lambda}\right) \Lambda_{\Omega}\left(F^{\lambda}\right)$ for any $\lambda \in \Lambda$.

Lemma 9.17. $\nabla$ is nonsingular, positive and self-adjoint.

Proof. If $\nabla \xi=0$, then, by definition, $F^{\lambda} \xi=0$ for all $\lambda \in \Lambda$. This implies $\xi=0$, since $\sum_{\lambda \in \Lambda} F^{\lambda}=1$. Because all $F^{\lambda}$ 's are projections, $\nabla$ is positive. Finally, to prove that $\nabla$ is self-adjoint, take any $\xi \in H_{\Omega}$. Then, since $\sum_{\lambda \in \Lambda} F^{\lambda}=1$ again, we get

$$
\sum_{\lambda \in \Lambda} \frac{\left\|F^{\lambda} \xi\right\|_{2}^{2}}{\left(1+n_{\lambda^{-1}} / n_{\lambda}\right)^{2}} \leq \sum_{\lambda \in \Lambda}\left\|F^{\lambda} \xi\right\|_{2}^{2}=\|\xi\|_{2}^{2}<\infty
$$

Hence

$$
\eta:=\sum_{\lambda \in \Lambda} \frac{1}{1+n_{\lambda^{-1}} / n_{\lambda}} F^{\lambda} \xi
$$

converges in norm in $H_{\Omega}$. We have

$$
F^{\lambda} \eta=\frac{1}{1+n_{\lambda^{-1}} / n_{\lambda}} F^{\lambda} \xi
$$

for any $\lambda \in \Lambda$. So

$$
\sum_{\lambda \in \Lambda}\left[\frac{n_{\lambda^{-1}}}{n_{\lambda}}\right]^{2}\left\|F^{\lambda} \eta\right\|_{2}^{2}=\sum_{\lambda \in \Lambda}\left[\frac{n_{\lambda^{-1}}}{n_{\lambda}}\right]^{2} \frac{\left\|F^{\lambda} \xi\right\|_{2}^{2}}{\left(1+n_{\lambda^{-1}} / n_{\lambda}\right)^{2}} \leq \sum_{\lambda \in \Lambda}\left\|F^{\lambda} \xi\right\|_{2}^{2}=\|\xi\|_{2}^{2}<\infty .
$$

It follows that $\eta \in \mathfrak{D}(\nabla)$. Moreover,

$$
\begin{aligned}
(1+\nabla) \eta & =\eta+\sum_{\lambda \in \Lambda} \frac{n_{\lambda^{-1}}}{n_{\lambda}} F^{\lambda} \eta \\
& =\sum_{\lambda \in \Lambda} \frac{1}{1+n_{\lambda^{-1}} / n_{\lambda}} F^{\lambda} \xi+\sum_{\lambda \in \Lambda} \frac{n_{\lambda^{-1}}}{n_{\lambda}} \frac{1}{1+n_{\lambda^{-1}} / n_{\lambda}} F^{\lambda} \xi \\
& =\sum_{\lambda \in \Lambda} F^{\lambda} \xi=\xi .
\end{aligned}
$$

Therefore, $(1+\nabla) \mathfrak{D}(\nabla)=H_{\Omega}$. By [19, Lemma 9.5], $\nabla$ is self-adjoint. 
It can be easily checked that, for any Borel function $g$ on the interval $[0, \infty)$, the linear operator $g(\nabla)$ (i.e., the Borel functional calculus of $\nabla$ under $g$ ) is given by

$$
g(\nabla)=\sum_{\lambda \in \Lambda} g\left(n_{\lambda^{-1}} / n_{\lambda}\right) F^{\lambda}
$$

with

$$
\mathfrak{D}(g(\nabla))=\left\{\xi \in H_{\Omega}: \sum_{\lambda \in \Lambda}\left|g\left(n_{\lambda^{-1}} / n_{\lambda}\right)\right|^{2}\left\|F^{\lambda} \xi\right\|^{2}<\infty\right\} .
$$

Lemma 9.18. The densely defined conjugate-linear map $S_{0}: H_{\Omega} \rightarrow H_{\Omega}$ defined on $\Lambda_{\Omega}(\mathcal{I}(\mathcal{R}, \mathcal{S}))$ by

$$
S_{0} \Lambda_{\Omega}(F):=\Lambda_{\Omega}\left(F^{\sharp}\right) \quad(F \in \mathcal{I}(\mathcal{R}, \mathcal{S}))
$$

is preclosed. The closure $S$ of $S_{0}$ has polar decomposition $\mathbf{J} \nabla^{1 / 2}$.

Proof. As remarked just before this lemma, we have

$$
\begin{aligned}
\mathfrak{D}\left(\nabla^{1 / 2}\right) & =\left\{\xi \in H_{\Omega}: \sum_{\lambda \in \Lambda} \frac{n_{\lambda^{-1}}}{n_{\lambda}}\left\|F^{\lambda} \xi\right\|^{2}\right\}, \\
\nabla^{1 / 2} \xi & =\sum_{\lambda \in \Lambda}\left[\frac{n_{\lambda^{-1}}}{n_{\lambda}}\right]^{1 / 2} F^{\lambda} \xi \quad\left(\forall \xi \in \mathfrak{D}\left(\nabla^{1 / 2}\right)\right) .
\end{aligned}
$$

Let $F \in \mathcal{I}(\mathcal{R}, \mathcal{S})$. With $\Xi^{*}(f)=F$, we have $\Lambda_{\Omega}(F)=\sum_{\lambda \in \Lambda} f(\lambda) \Lambda_{\Omega}\left(F^{\lambda}\right)$. So $F^{\lambda} \Lambda_{\Omega}(F)=f(\lambda) \Lambda_{\Omega}\left(F^{\lambda}\right)$ for all $\lambda \in \Lambda$. From this, we obtain

$$
\begin{aligned}
\sum_{\lambda \in \Lambda} \frac{n_{\lambda^{-1}}}{n_{\lambda}}\left\|F^{\lambda} \Lambda_{\Omega}(F)\right\|^{2} & =\sum_{\lambda \in \Lambda} \frac{n_{\lambda^{-1}}}{n_{\lambda}}|f(\lambda)|^{2} n_{\lambda}=\sum_{\lambda \in \Lambda} n_{\lambda^{-1}}|f(\lambda)|^{2} \leq\|f\|_{\infty}\|F\|_{1, r} \\
& <\infty .
\end{aligned}
$$

Thus $\Lambda_{\Omega}(F)$ belongs to $\mathfrak{D}\left(\nabla^{1 / 2}\right)$, and

This in turn implies

$$
\nabla^{1 / 2} \Lambda_{\Omega}(F)=\sum_{\lambda \in \Lambda}\left[\frac{n_{\lambda^{-1}}}{n_{\lambda}}\right]^{1 / 2} f(\lambda) \Lambda_{\Omega}\left(F^{\lambda}\right) .
$$

$$
\begin{aligned}
\mathbf{J} \nabla^{1 / 2} \Lambda_{\Omega}(F) & =\sum_{\lambda \in \Lambda}\left[\frac{n_{\lambda^{-1}}}{n_{\lambda}}\right]^{1 / 2} \overline{f(\lambda)} \mathbf{J} \Lambda_{\Omega}\left(F^{\lambda}\right) \\
& =\sum_{\lambda \in \Lambda}\left[\frac{n_{\lambda^{-1}}}{n_{\lambda}}\right]^{1 / 2} \overline{f(\lambda)}\left[\frac{n_{\lambda}}{n_{\lambda}-1}\right]^{1 / 2} \Lambda_{\Omega}\left(F^{\lambda^{-1}}\right) \\
& =\sum_{\lambda \in \Lambda} \overline{f(\lambda)} \Lambda_{\Omega}\left(F^{\lambda^{-1}}\right)=\sum_{\lambda \in \Lambda} \overline{f\left(\lambda^{-1}\right)} \Lambda_{\Omega}\left(F^{\lambda}\right)=\Lambda_{\Omega}\left(F^{\sharp}\right)=S_{0} \Lambda_{\Omega}(F) .
\end{aligned}
$$

Therefore, $S_{0}$ is preclosed, and its closure $S$ satisfies $S \subseteq \mathbf{J} \nabla^{1 / 2}$. 
In order to prove that $S=\mathbf{J} \nabla^{1 / 2}$, it suffices to show that $\Lambda_{\Omega}(\mathcal{I}(\mathcal{R}, \mathcal{S}))$ is a core for $\nabla^{1 / 2}$. For this purpose, suppose that $\xi \in \mathfrak{D}\left(\nabla^{1 / 2}\right)$ satisfies

$$
\left(\xi \mid \Lambda_{\Omega}\left(F^{\lambda}\right)\right)+\left(\nabla^{1 / 2} \xi \mid \nabla^{1 / 2} \Lambda_{\Omega}\left(F^{\lambda}\right)\right)=0
$$

for all $\lambda \in \Lambda$. Then

$$
\begin{aligned}
0 & =\left(\xi \mid \Lambda_{\Omega}\left(F^{\lambda}\right)\right)+\left(\sum_{\lambda_{1} \in \Lambda}\left[\frac{n_{\lambda_{1}^{-1}}}{n_{\lambda_{1}}}\right]^{1 / 2} F^{\lambda_{1}} \xi \mid\left[\frac{n_{\lambda^{-1}}}{n_{\lambda}}\right]^{1 / 2} \Lambda_{\Omega}\left(F^{\lambda}\right)\right) \\
& =\left(\xi \mid \Lambda_{\Omega}\left(F^{\lambda}\right)\right)+\frac{n_{\lambda^{-1}}}{n_{\lambda}}\left(\xi \mid \Lambda_{\Omega}\left(F^{\lambda}\right)\right) .
\end{aligned}
$$

Thus $\left(\xi \mid \Lambda_{\Omega}\left(F^{\lambda}\right)\right)=0$ for all $\lambda \in \Lambda$. Since $\left\{\Lambda_{\Omega}\left(F^{\lambda}\right): \lambda \in \Lambda\right\}$ is total in $H_{\Omega}$, we have $\xi=0$. It follows that the subspace $\Lambda_{\Omega}\left(\mathcal{I}_{0}(\mathcal{R}, \mathcal{S})\right)$ is already a core for $\nabla^{1 / 2}$.

Corollary 9.19. The subspace $\Lambda_{\Omega}\left(\mathcal{I}_{0}(\mathcal{R}, \mathcal{S})\right)$ is a core for $\nabla^{r}$ for all $r \in \mathbb{R}$. Moreover, $\nabla^{r} \Lambda_{\Omega}\left(\mathcal{I}_{0}(\mathcal{R}, \mathcal{S})\right)=\Lambda_{\Omega}\left(\mathcal{I}_{0}(\mathcal{R}, \mathcal{S})\right)$ for any $r \in \mathbb{R}$.

Proof. Let $r \in \mathbb{R}$. We know that

$$
\begin{aligned}
\mathfrak{D}\left(\nabla^{r}\right) & =\left\{\xi \in H_{\Omega}: \sum_{\lambda \in \Lambda}\left[\frac{n_{\lambda^{-1}}}{n_{\lambda}}\right]^{2 r}\left\|F^{\lambda} \xi\right\|^{2}\right\}, \\
\nabla^{r} \xi & =\sum_{\lambda \in \Lambda}\left[\frac{n_{\lambda^{-1}}}{n_{\lambda}}\right]^{r} F^{\lambda} \xi \quad\left(\forall \xi \in \mathfrak{D}\left(\nabla^{r}\right)\right) .
\end{aligned}
$$

So it is obvious that $\Lambda_{\Omega}\left(\mathcal{I}_{0}(\mathcal{R}, \mathcal{S})\right) \in \mathfrak{D}\left(\nabla^{r}\right)$. In order to show that $\Lambda_{\Omega}\left(\mathcal{I}_{0}(\mathcal{R}, \mathcal{S})\right)$ is a core for $\nabla^{r}$, suppose that, for a vector $\xi \in \mathfrak{D}\left(\nabla^{r}\right)$, equation 9.8 holds except that, this time, the power " $1 / 2$ " in the equation is replaced by $r$. Then, by exactly the same argument as in Lemma 9.18, we arrive at the conclusion that $\xi=0$.

The last assertion easily follows from the fact that $\nabla^{r} \Lambda_{\Omega}\left(F^{\lambda}\right)=$ $\left[n_{\lambda^{-1}} / n_{\lambda}\right]^{r} \Lambda_{\Omega}\left(F^{\lambda}\right)$ for any $\lambda \in \Lambda$.

Theorem 9.20. Define a product and an involution $\sharp$ on $\Lambda_{\Omega}(\mathcal{I}(\mathcal{R}, \mathcal{S}))$ by

$$
\Lambda_{\Omega}\left(F_{1}\right) \Lambda_{\Omega}\left(F_{2}\right):=\Lambda_{\Omega}\left(F_{1} * F_{2}\right), \quad \Lambda_{\Omega}\left(F_{1}\right)^{\sharp}:=\Lambda_{\Omega}\left(F_{1}^{\sharp}\right) \quad\left(F_{1}, F_{2} \in \mathcal{I}(\mathcal{R}, \mathcal{S})\right) .
$$

Then the subspace $\mathfrak{A}(\mathcal{R}, \mathcal{S}):=\Lambda_{\Omega}(\mathcal{I}(\mathcal{R}, \mathcal{S})$ ) with these operations becomes a left Hilbert algebra in $H_{\Omega}$ whose modular operator and modular conjugation are $\nabla$ and J defined before.

Proof. It is clear that $\mathfrak{A}(\mathcal{R}, \mathcal{S})$ is an involutive algebra over $\mathbb{C}$ which is dense in the Hilbert space $H_{\Omega}$.

For each $F \in \mathcal{I}(\mathcal{R}, \mathcal{S})$, the mapping $\Lambda_{\Omega}\left(F_{1}\right) \mapsto \Lambda_{\Omega}(F) \Lambda_{\Omega}\left(F_{1}\right)$ is bounded, due to Lemma 9.16 . 
By Lemma 9.16 again, we have

$$
\left(\Lambda_{\Omega}(F) \Lambda_{\Omega}\left(F_{1}\right) \mid \Lambda_{\Omega}\left(F_{2}\right)\right)=\left(\Lambda_{\Omega}\left(F_{1}\right) \mid \Lambda_{\Omega}(F)^{\sharp} \Lambda_{\Omega}\left(F_{2}\right)\right) .
$$

Thanks to Lemma 9.18, the involution $\Lambda_{\Omega}(F) \mapsto \Lambda_{\Omega}(F)^{\sharp}$ is preclosed.

Recall the element $F^{0}$ mentioned just before Theorem 9.11. Since $\Lambda_{\Omega}\left(F^{0}\right)$ is the identity for $\mathfrak{A}(\mathcal{R}, \mathcal{S})$, we see that the linear span $(\mathfrak{A}(\mathcal{R}, \mathcal{S}))^{2}$ of the set $\left\{\Lambda_{\Omega}\left(F_{1}\right) \Lambda_{\Omega}\left(F_{2}\right): F_{1}, F_{2} \in \mathcal{I}(\mathcal{R}, \mathcal{S})\right\}$ equals $\mathfrak{A}(\mathcal{R}, \mathcal{S})$.

Therefore, $\mathfrak{A}(\mathcal{R}, \mathcal{S})$ is a left Hilbert algebra in $H_{\Omega}$. By Lemma 9.18, the modular operator and the modular conjugation associated with $\mathfrak{A}(\mathcal{R}, \mathcal{S})$ are $\nabla$ and $\mathbf{J}$, respectively.

Definition 9.21. We call the left von Neumann algebra of the left Hilbert algebra $\mathfrak{A}(\mathcal{R}, \mathcal{S})$ the Hecke von Neumann algebra associated with the Hecke pair $(\mathcal{R}, \mathcal{S})$, and denote it by $\mathcal{H}^{*}(\mathcal{R}, \mathcal{S})$. Hence

$$
\mathcal{H}^{*}(\mathcal{R}, \mathcal{S})=\pi_{\ell}(\mathcal{I}(\mathcal{R}, \mathcal{S}))^{\prime \prime}=\sigma \text {-strong }{ }^{*} \text { closure of } \pi_{\ell}(\mathcal{I}(\mathcal{R}, \mathcal{S})) .
$$

Proposition 9.22. The involutive subalgebra $\mathfrak{A}_{0}(\mathcal{R}, \mathcal{S}):=\Lambda_{\Omega}\left(\mathcal{I}_{0}(\mathcal{R}, \mathcal{S})\right.$ ) of $\mathfrak{A}(\mathcal{R}, \mathcal{S})$ is equivalent to $\mathfrak{A}(\mathcal{R}, \mathcal{S})$ as a left Hilbert algebra, that is, $\mathfrak{A}_{0}(\mathcal{R}, \mathcal{S})^{\prime \prime}=$ $\mathfrak{A}(\mathcal{R}, \mathcal{S})^{\prime \prime}$. In particular, $\mathcal{H}^{*}(\mathcal{R}, \mathcal{S})=\pi_{\ell}\left(\mathfrak{A}_{0}(\mathcal{R}, \mathcal{S})\right)^{\prime \prime}$.

Proof. It suffices to prove that the $S$-operator of $\mathfrak{A}_{0}(\mathcal{R}, \mathcal{S})$ equals $S=\mathbf{J} \nabla^{1 / 2}$. But this follows from the fact that $\mathfrak{A}_{0}(\mathcal{R}, \mathcal{S})$ is a core for $\nabla^{1 / 2}$, verified in the proof of Lemma 9.18

Lemma 9.23. The left Hilbert algebra $\mathfrak{A}_{0}(\mathcal{R}, \mathcal{S})$ is a core for the operator $S^{*}$. Moreover,

$$
S^{*} \Lambda_{\Omega}\left(F^{\lambda}\right)=\frac{n_{\lambda}}{n_{\lambda^{-1}}} \Lambda_{\Omega}\left(F^{\lambda^{-1}}\right)
$$

for any $\lambda \in \Lambda$. In particular, $\mathfrak{A}_{0}(\mathcal{R}, \mathcal{S})$ is invariant under $S^{*}$.

Proof. The first assertion follows from Corollary 9.19 and the fact that $\mathfrak{D}\left(S^{*}\right)=$ $\mathfrak{D}\left(\nabla^{-1 / 2}\right)$. The second follows by a direct computation, using $S^{*}=\mathbf{J} \nabla^{-1 / 2}$.

By convention, we write $\eta^{b}$ for $S^{*} \eta$.

Proposition 9.24. The algebra $\mathfrak{A}_{0}(\mathcal{R}, \mathcal{S})$ is also a right Hilbert algebra with involution b contained in $\mathfrak{A}(\mathcal{R}, \mathcal{S})^{\prime}$, and is equivalent to $\mathfrak{A}(\mathcal{R}, \mathcal{S})^{\prime}$ as a right Hilbert algebra.

Proof. From Lemma 9.16, every element of $\mathfrak{A}_{0}(\mathcal{R}, \mathcal{S})$ is a right bounded vector with respect to the left Hilbert algebra $\mathfrak{A}(\mathcal{R}, \mathcal{S})$. It also belongs to $\mathfrak{D}\left(S^{*}\right)$ by Lemma 9.23 Hence $\mathfrak{A}_{0}(\mathcal{R}, \mathcal{S}) \subseteq \mathfrak{A}(\mathcal{R}, \mathcal{S})^{\prime}$. Therefore, $\mathfrak{A}_{0}(\mathcal{R}, \mathcal{S})$ is a right Hilbert 
algebra in $H_{\Omega}$. Because $\mathfrak{A}_{0}(\mathcal{R}, \mathcal{S})$ is a core for $S^{*}$, it follows that it is equivalent to $\mathfrak{A}(\mathcal{R}, \mathcal{S})^{\prime}$.

Corollary 9.25. Let $F^{0} \in \mathcal{I}(\mathcal{R}, \mathcal{S})$ be as before. Then $\zeta_{0}:=\Lambda_{\Omega}\left(F^{0}\right)$ is a cyclic and separating vector for $\mathcal{H}^{*}(\mathcal{R}, \mathcal{S})$.

Proof. Since $\zeta_{0}$ is the identity of $\mathfrak{A}_{0}(\mathcal{R}, \mathcal{S})$, it follows from Proposition 9.24 that

$$
\begin{aligned}
& H_{\Omega}=\overline{\mathfrak{A}_{0}(\mathcal{R}, \mathcal{S})}=\overline{\pi_{\ell}\left(\mathfrak{A}_{0}(\mathcal{R}, \mathcal{S})\right) \zeta_{0}} \subseteq \overline{\mathcal{H}^{*}(\mathcal{R}, \mathcal{S}) \zeta_{0}} \\
& H_{\Omega}=\overline{\mathfrak{A}_{0}(\mathcal{R}, \mathcal{S})}=\overline{\pi_{r}\left(\mathfrak{A}_{0}(\mathcal{R}, \mathcal{S})\right) \zeta_{0}} \subseteq \overline{\mathcal{H}^{*}(\mathcal{R}, \mathcal{S})^{\prime} \zeta_{0}}
\end{aligned}
$$

Hence $\zeta_{0}$ is cyclic for both $\mathcal{H}^{*}(\mathcal{R}, \mathcal{S})$ and $\mathcal{H}^{*}(\mathcal{R}, \mathcal{S})^{\prime}$. Thus we are done.

Corollary 9.26. We have $\mathfrak{A}(\mathcal{R}, \mathcal{S})^{\prime \prime}=\mathcal{H}^{*}(\mathcal{R}, \mathcal{S}) \zeta_{0}$ and $\mathfrak{A}(\mathcal{R}, \mathcal{S})^{\prime}=\mathcal{H}^{*}(\mathcal{R}, \mathcal{S})^{\prime} \zeta_{0}$.

Proof. It is clear that $\mathfrak{A}(\mathcal{R}, \mathcal{S})$ is a left Hilbert subalgebra of the full left Hilbert algebra $\mathcal{H}^{*}(\mathcal{R}, \mathcal{S}) \zeta_{0}$. Hence $\mathfrak{A}(\mathcal{R}, \mathcal{S})^{\prime \prime} \subseteq\left(\mathcal{H}^{*}(\mathcal{R}, \mathcal{S}) \zeta_{0}\right)^{\prime \prime}=\mathcal{H}^{*}(\mathcal{R}, \mathcal{S}) \zeta_{0}$. On the other hand, by Proposition 9.24 , $\zeta_{0}$ is the identity for the right Hilbert algebra $\mathfrak{A}(\mathcal{R}, \mathcal{S})^{\prime}$. This implies that $\mathfrak{A}(\mathcal{R}, \mathcal{S})^{\prime} \subseteq \mathcal{H}^{*}(\mathcal{R}, \mathcal{S})^{\prime} \zeta_{0}$. Hence,

$$
\mathcal{H}^{*}(\mathcal{R}, \mathcal{S}) \zeta_{0}=\left(\mathcal{H}^{*}(\mathcal{R}, \mathcal{S}) \zeta_{0}\right)^{\prime \prime}=\left(\mathcal{H}^{*}(\mathcal{R}, \mathcal{S})^{\prime} \zeta_{0}\right)^{\prime} \subseteq\left(\mathfrak{A}(\mathcal{R}, \mathcal{S})^{\prime}\right)^{\prime}=\mathfrak{A}(\mathcal{R}, \mathcal{S})^{\prime \prime}
$$

Therefore, $\mathfrak{A}(\mathcal{R}, \mathcal{S})^{\prime \prime}=\mathcal{H}^{*}(\mathcal{R}, \mathcal{S}) \zeta_{0}$.

Corollary 9.27. The faithful normal semifinite weight $\varphi_{0}$ on $\mathcal{H}^{*}(\mathcal{R}, \mathcal{S})$ associated with the left Hilbert algebra $\mathfrak{A}(\mathcal{R}, \mathcal{S})$ is the normal state $\varphi_{0}$ given by

$$
\varphi_{0}(T)=\left(T \zeta_{0} \mid \zeta_{0}\right) \quad\left(\forall T \in \mathcal{H}^{*}(\mathcal{R}, \mathcal{S})\right) .
$$

Proof. This follows from Corollary 9.26 .

Remark. It is easy to check that the 1-cocycle $c$ defined by 9.2 is equal to $\sum_{\lambda \in \Lambda}\left(n_{\lambda^{-1}} / n_{\lambda}\right) \chi_{\mathcal{C}_{\lambda}}$ up to a null set. Hence $\nabla$ is characterized by

$$
\nabla \xi=\Phi(c) \xi \quad(\xi \in \mathfrak{D}(\nabla)),
$$

where we extend $\Phi$ to a map from $\mathcal{S}$-biinvariant functions on $\mathcal{R}$ to $\mathcal{S}$-invariant functions on $I \times \mathcal{R}$.

\section{§10. Relation between $\mathcal{H}^{*}(\mathcal{R}, \mathcal{S})$ and $\widehat{M}$}

Throughout this section, we assume that $(\mathcal{R}, \mathcal{S})$ is a Hecke pair, and retain most of the notation introduced previously.

Lemma 10.1. Let $R=\left[R_{i, j}(x)\right]_{i, j \in I, x \in X}$ be in $\widehat{M}$ and $F_{1}, F_{2}$ be in $\mathcal{I}(\mathcal{R}, \mathcal{S})$. Then $R *\left(F_{1} * F_{2}\right)=\left(R * F_{1}\right) * F_{2}$ in $\mathfrak{n}_{\Omega}$. 
Proof. By definition, there exists a $\mu$-null subset $N_{0}$ of $X$ such that

$$
\begin{aligned}
& R_{i, \sigma(x, y)(j)}(x)=R_{\sigma(y, x)(i), j}(y) \\
& \quad \text { for all }(x, y) \in \mathcal{R}_{0}:=\mathcal{R} \cap\left(N_{0}^{c} \times N_{0}^{c}\right) \text { and all } i, j \in I .
\end{aligned}
$$

On the other hand, there exists a $\mu$-null subset $N_{1}$ of $X$ such that

$$
\sum_{i \in I}\left|F_{k}(i, x)\right|=\left\|F_{k}\right\|_{1, \ell} \quad \text { for all } x \in N_{1}^{c}, i \in I \text { and } k=1,2 .
$$

Put $N_{2}:=\bigcup_{i \in I} \psi_{i}^{-1}\left(N_{0} \cup N_{1}\right)$ and $\mathcal{R}_{1}:=\mathcal{R} \cap\left(N_{1}^{2} \times N_{2}^{c}\right)$. Let $i \in I$ and $x \in N_{2}^{c}$.

Then

$$
\begin{aligned}
\left(R *\left(F_{1} * F_{2}\right)\right)(i, x) & =\sum_{j \in I} R_{i, j}(x)\left(F_{1} * F_{2}\right)(j, x) \\
& =\sum_{j \in I} R_{i, j}(x)\left(\sum_{k \in I} F_{1}\left(\sigma\left(\psi_{k}(x), x\right)(j), \psi_{k}(x)\right) F_{2}(k, x)\right) .
\end{aligned}
$$

Here we remark that

$$
\begin{aligned}
\sum_{j, k \in I}\left|R_{i, j}(x)\right| \mid F_{1}(\sigma & \left.\left(\psi_{k}(x), x\right)(j), \psi_{k}(x)\right)|| F_{2}(k, x) \mid \\
& \leq\|R\| \sum_{k \in I}\left(\sum_{j \in I}\left|F_{1}\left(\sigma\left(\psi_{k}(x), x\right)(j), \psi_{k}(x)\right)\right|\right)\left|F_{2}(k, x)\right| \\
& =\|R\|\left\|F_{1}\right\|_{1, \ell}\left\|F_{2}\right\|_{1, \ell}<\infty .
\end{aligned}
$$

Hence, by Fubini's theorem, we have

$$
\begin{aligned}
\left(R *\left(F_{1} * F_{2}\right)\right)(i, x) & =\sum_{k \in I}\left(\sum_{j \in I} R_{i, j}(x) F_{1}\left(\sigma\left(\psi_{k}(x), x\right)(j), \psi_{k}(x)\right)\right) F_{2}(k, x) \\
& =\sum_{k \in I}\left(\sum_{j \in I} R_{i, \sigma\left(x, \psi_{k}(x)\right)(j)}(x) F_{1}\left(j, \psi_{k}(x)\right)\right) F_{2}(k, x) \\
& =\sum_{k \in I}\left(\sum_{j \in I} R_{\sigma\left(\psi_{k}(x), x\right)(i), j}\left(\psi_{k}(x)\right) F_{1}\left(j, \psi_{k}(x)\right)\right) F_{2}(k, x) \\
& =\sum_{k \in I}\left(R * F_{1}\right)\left(\sigma\left(\psi_{k}(x), x\right)(i), \psi_{k}(x)\right) F_{2}(k, x) \\
& =\left(\left(R * F_{1}\right) * F_{2}\right)(i, x) .
\end{aligned}
$$

Theorem 10.2. The Hecke von Neumann algebra $\mathcal{H}^{*}(\mathcal{R}, \mathcal{S})$ exactly equals $\hat{\lambda}(\widehat{M})$. Therefore, $\widehat{M}$ is *-isomorphic to $\mathcal{H}^{*}(\mathcal{R}, \mathcal{S})$. 
Proof. Let $\lambda \in \Lambda$. As in the proof of Lemma 8.6, consider the "matrix" $R^{\lambda}:=$ $\left[R_{i, j}^{\lambda}(x)\right]_{i, j \in I, x \in X}$ in $\widehat{M}$ given by

$$
R_{i, j}^{\lambda}(x)=F^{\lambda}\left(\sigma\left(\psi_{j}(x), x\right)(i), \psi_{j}(x)\right) \quad(i, j \in I, x \in X) .
$$

Then, for any $F \in \mathcal{I}(\mathcal{R}, \mathcal{S})$, with the notation of Section 7, we have

$$
\begin{aligned}
\left(R^{\lambda} * F\right)(i, x) & =\sum_{j \in I} R_{i, j}^{\lambda}(x) F(j, x)=\sum_{j \in I} F^{\lambda}\left(\sigma\left(\psi_{j}(x), x\right)(i), \psi_{j}(x)\right) F(j, x) \\
& =\left(F^{\lambda} * F\right)(i, x) .
\end{aligned}
$$

Thus $R^{\lambda} * F=F^{\lambda} * F$. From this, we obtain

$$
\hat{\lambda}\left(R^{\lambda}\right) \Lambda_{\Omega}(F)=\Lambda_{\Omega}\left(R^{\lambda} * F\right)=\Omega_{\Omega}\left(F^{\lambda} * F\right)=\pi_{\ell}\left(F^{\lambda}\right) \Lambda_{\Omega}(F) .
$$

Hence $\hat{\lambda}\left(R^{\lambda}\right)=\pi_{\ell}\left(F^{\lambda}\right)$ for any $\lambda \in \Lambda$. It follows that $\pi_{\ell}\left(\mathcal{I}_{0}(\mathcal{R}, \mathcal{S})\right)$ is contained in $\hat{\lambda}(\widehat{M})$. Since $\mathcal{H}^{*}(\mathcal{R}, \mathcal{S})=\pi_{\ell}\left(\mathcal{I}_{0}(\mathcal{R}, \mathcal{S})\right)^{\prime \prime}$ by Proposition 9.22 we conclude that $\mathcal{H}^{*}(\mathcal{R}, \mathcal{S}) \subseteq \hat{\lambda}(\widehat{M})$.

To prove the reverse inclusion, let us take any $R \in \widehat{M}$. Then, by Lemma 10.1 . for any $F_{1}, F_{2} \in \mathcal{I}_{0}(\mathcal{R}, \mathcal{S})$ we obtain

$$
\begin{aligned}
\hat{\lambda}(R) \pi_{r}\left(F_{1}\right) \Lambda_{\Omega}\left(F_{2}\right) & =\hat{\lambda}(R) \Lambda_{\Omega}\left(F_{2} * F_{1}\right)=\Lambda_{\Omega}\left(R *\left(F_{2} * F_{1}\right)\right)=\Lambda_{\Omega}\left(\left(R * F_{2}\right) * F_{1}\right) \\
& =\pi_{r}\left(F_{1}\right) \Lambda_{\Omega}\left(R * F_{2}\right)=\pi_{r}\left(F_{1}\right) \hat{\lambda}(R) \Lambda_{\Omega}\left(F_{2}\right) .
\end{aligned}
$$

So $\hat{\lambda}(R) \pi_{r}\left(F_{1}\right)=\pi_{r}\left(F_{1}\right) \hat{\lambda}(R)$ for all $F_{1} \in \mathcal{I}_{0}(\mathcal{R}, \mathcal{S})$. Hence, by Proposition 9.24 .

$$
\hat{\lambda}(R) \in \pi_{r}\left(\mathcal{I}_{0}(\mathcal{R}, \mathcal{S})\right)^{\prime}=\left(\mathcal{H}^{*}(\mathcal{R}, \mathcal{S})^{\prime}\right)^{\prime}=\mathcal{H}^{*}(\mathcal{R}, \mathcal{S}) .
$$

Therefore, $\hat{\lambda}(\widehat{M}) \subseteq \mathcal{H}^{*}(\mathcal{R}, \mathcal{S})$.

There is a notion of normality of a Borel subrelation of a discrete equivalence relation (see [8]). Normality of ergodic subrelations is studied intensively also in 3 . It is shown in 3 that $\mathcal{S}$ being normal in $\mathcal{R}$ implies that $(\mathcal{R}, \mathcal{S})$ is a Hecke pair.

Corollary 10.3. Suppose that $\mathcal{S}$ is normal in $\mathcal{R}$. Then the Hecke von Neumann algebra $\mathcal{H}^{*}(\mathcal{R}, \mathcal{S})$ is $*$-isomorphic to the group von Neumann algebra of the countable discrete group $\Gamma:=\mathcal{R} / \mathcal{S}$ (see [8], [3] for the definition of the group $\mathcal{R} / \mathcal{S}$ ).

Proof. By [3, there is a minimal coaction $\alpha$ of $\Gamma$ on $W^{*}(\mathcal{R})$ such that the fixedpoint algebra $W^{*}(\mathcal{R})^{\alpha}$ of $\alpha$ is equal to $W^{*}(\mathcal{S})$. It is well-known that, in this case, $\widehat{M}$ is $*$-isomorphic to the group von Neumann algebra $W^{*}(\Gamma)$ of $\Gamma$. By Theorem 10.2 , $\mathcal{H}^{*}(\mathcal{R}, \mathcal{S})$ is $*$-isomorphic to $W^{*}(\Gamma)$. 
Remark. In the above setting, it is easy to check that $M$ is $*$-isomorphic to $L^{\infty}(\Gamma)$, and the map $\mathcal{F}$ defined in Section 8 is given by $\Lambda_{\widehat{\Omega}}\left(\lambda_{\Gamma}(\gamma)\right) \mapsto \Lambda_{\Omega}\left(\delta_{\gamma}\right)$ for each $\gamma \in \Gamma$, where $\lambda_{\Gamma}$ is the left regular representation of $\Gamma$ on $L^{2}(\Gamma)$. This fact justifies our "Fourier transform" terminology for $\mathcal{F}$.

\section{§11. Relation to Hecke pairs of groups}

In this section, we discuss Hecke von Neumann algebras in the case where our ergodic discrete measured equivalence relations $\mathcal{S} \subseteq \mathcal{R}$ are derived from the "groupsubgroup" setting, whose meaning is explained in detail below.

As before, we start with an ergodic discrete measured equivalence relation $\mathcal{R}$ on a standard Borel probability space $(X, \mathfrak{B}, \mu)$ and an ergodic Borel subrelation $\mathcal{S}$ of $\mathcal{R}$. We also retain most of the notation introduced previously.

Suppose that there exist a Borel subrelation $\mathcal{P}$ contained in $\mathcal{S}$, a countable discrete group $G$ in the normalizer group $N[\mathcal{P}]$ of the full group $[\mathcal{P}]$ and a subgroup $H$ of $G$ such that

(1) $G \cap[\mathcal{P}]=\{e\}$, i.e., the action of $G$ on $\mathcal{P}$ is outer;

(2) $(\mathcal{S} \subseteq \mathcal{R})=(H \ltimes \mathcal{P} \subseteq G \ltimes \mathcal{P})$;

(3) $(G, H)$ is a Hecke pair of groups, i.e., $G=\left\{g \in G:\left[H: H \cap g^{-1} H g\right]<\infty\right\}$;

(4) the intermediate subrelation $\mathcal{P}_{g}:=\left(H \cap g^{-1} H g\right) \ltimes \mathcal{P}$ is ergodic for each $g \in G$.

Thus we have

$$
\begin{aligned}
\mathcal{S} & =\{(x, y) \in X \times X: \exists h \in H,(x, h(y)) \in \mathcal{P}\}, \\
\mathcal{R} & =\{(x, y) \in X \times X: \exists g \in G,(x, g(y)) \in \mathcal{P}\} .
\end{aligned}
$$

In this setting, it is known (see [13, Example 3.5(i)] and 3, Section 9]) that $(\mathcal{R}, \mathcal{S})$ is a Hecke pair.

Let $\left\{t_{q}\right\}_{q \in H \backslash G} \subseteq G$ be a set of representatives of the right coset space $H \backslash G$ with $t_{H}=e$. We also let $\left\{q_{\lambda} \in H \backslash G: \lambda \in H \backslash G / H\right\}$ be a complete set of representatives of the double coset space $\Lambda:=H \backslash G / H$ satisfying $q_{H}=H \in H \backslash G$. We simply write $t_{\lambda}$ for $t_{q_{\lambda}}$ for each $\lambda \in \Lambda=H \backslash G / H$.

For any $g \in G$, put $L(g):=\left[H: H \cap g^{-1} H g\right]$. Because $(G, H)$ is a Hecke pair, $L(g)$ is finite for all $g \in G$. Note that the function $L: G \rightarrow \mathbb{N}$ is two-sided $H$ invariant, so it may be viewed as a function on $H \backslash G / H$. By definition, we find that, for any $\lambda \in \Lambda=H \backslash G / H, L\left(t_{\lambda}\right)$ equals $\left|H_{q_{\lambda}} \backslash H\right|$, where $H_{q_{\lambda}}$ is the stabilizer group at the point $q_{\lambda}$ under the $H$-action $(q, h) \in H \backslash G \times H \mapsto q \cdot h \in H \backslash G$. For any $\lambda \in \Lambda$, we choose a set $\left\{h_{i}^{(\lambda)}: 1 \leq i \leq L\left(t_{\lambda}\right)\right\} \subseteq H$ of representatives of the quotient space $H_{q_{\lambda}} \backslash H$. Then, by construction, the points $q_{\lambda} h_{i}^{(\lambda)}\left(\lambda \in H \backslash G / H, 1 \leq i \leq L\left(t_{\lambda}\right)\right)$ are all distinct and $H \backslash G=\left\{q_{\lambda} h_{i}^{(\lambda)}: \lambda \in H \backslash G / H, 1 \leq i \leq L\left(t_{\lambda}\right)\right\}$. 
For each $q \in H \backslash G$, set $\psi_{q}:=t_{q}$. We see that $\left\{\psi_{q}\right\}_{q \in H \backslash G}$ is a set of choice functions for $\mathcal{S} \subseteq \mathcal{R}$. According to the description $H \backslash G=\left\{q_{\lambda} h_{i}^{(\lambda)}: \lambda \in \Lambda, 1 \leq\right.$ $\left.i \leq L\left(t_{\lambda}\right)\right\}$ stated in the previous paragraph, the choice functions $\left\{\psi_{q}\right\}$ can be relabeled in the form $\left\{\psi_{\lambda, n}: \lambda \in \Lambda, n=1, \ldots, n_{\lambda}\right\}$, where $\psi_{\lambda, n}:=t_{\lambda} h_{n}^{(\lambda)}$ and $n_{\lambda}:=L\left(t_{\lambda}\right)$. These are exactly Aoi's choice functions for $\mathcal{S} \subseteq \mathcal{R}$ (see [2]). Hence the factor map $\Xi$ is a map from $H \backslash G \times X$ onto $\Lambda=H \backslash G / H$.

By outerness of the action of $G$, we may and do assume that, for each $(x, y) \in \mathcal{R}$, the mapping $\sigma(x, y): H \backslash G \rightarrow H \backslash G$ is the right translation $q \in$ $H \backslash G \mapsto q g \in H \backslash G$ by $g \in G$, where $g$ is determined by the condition $(g(x), y) \in \mathcal{P}$. In particular, $\mathcal{P}$ is included in $\operatorname{Ker}(\sigma)$. From this and the ergodicity of $\mathcal{P}_{g}$, it follows that each $F \in L^{\infty}((H \backslash G) \times X)^{\mathcal{S}}$ depends only on the first variable, so that there exists a unique function $f \in \ell^{\infty}(H \backslash G)$ such that $F(q, x)=f(q)$ for a.e. $x \in X$ and all $q \in H \backslash G$. Moreover, $\mathcal{S}$-invariance of $F$ entails that $f$ is $H$-invariant. Thus $f$ is regarded as an element of $\ell^{\infty}(H \backslash G / H)$. With the notation introduced before, we have $\Xi^{*}(f)=F$. We shall freely identify functions on $H \backslash G$ with those on $G$ which are right $H$-invariant, and functions on $H \backslash G / H$ with those on $G$ which are $H$-biinvariant.

The faithful normal semifinite weight $\Omega$ on $L^{\infty}((H \backslash G) \times X)^{\mathcal{S}}=\ell^{\infty}(H \backslash G / H)$ is given by

$$
\Omega(f)=\sum_{q \in H \backslash G} f(q)=\sum_{\lambda \in \Lambda} f(\lambda) n_{\lambda}
$$

for any $f \in L^{\infty}((H \backslash G) \times X)_{+}^{\mathcal{S}}=\ell^{\infty}(H \backslash G / H)_{+}$. We have

$$
\begin{aligned}
\mathfrak{n}_{\Omega} & =\left\{f \in \ell^{\infty}(H \backslash G / H): \sum_{q \in H \backslash G}|f(q)|^{2}=\sum_{\lambda \in \Lambda}|f(\lambda)|^{2} n_{\lambda}<\infty\right\}, \\
\mathcal{I}(\mathcal{R}, \mathcal{S}) & =\left\{f \in \ell^{\infty}(H \backslash G / H): \sum_{\lambda \in \Lambda}|f(\lambda)| n_{\lambda}<\infty, \sum_{\lambda \in \Lambda}\left|f\left(\lambda^{-1}\right)\right| n_{\lambda}<\infty\right\}, \\
\mathcal{I}_{0}(\mathcal{R}, \mathcal{S}) & =\left\{f \in \ell^{\infty}(H \backslash G / H): f \text { has finite support }\right\} .
\end{aligned}
$$

Let $f_{1}, f_{2} \in \mathcal{I}(\mathcal{R}, \mathcal{S})$. Since $\sigma\left(\psi_{q}(x), x\right)(q)=q t_{q}^{-1}$ in our setting, the convolution $f_{1} * f_{2}$ in $\mathcal{I}(\mathcal{R}, \mathcal{S})$ is given by

$$
\left(f_{1} * f_{2}\right)(q)=\sum_{p \in H \backslash G} f_{1}\left(q t_{p}^{-1}\right) f_{2}(p) .
$$

As a function on $G$, this is the same as

$$
\left(f_{1} * f_{2}\right)(g)=\sum_{p \in H \backslash G} f_{1}\left(g t_{p}^{-1}\right) f_{2}\left(t_{p}\right) \quad(g \in G) .
$$

So $\mathcal{I}_{0}(\mathcal{R}, \mathcal{S})$ is exactly the Hecke algebra associated with the Hecke pair $(G, H)$ in the theory of automorphic forms. Because $\sigma\left(\psi_{q}(x), x\right)(H)=H t_{q}^{-1}$, the involu- 
tion $f^{\sharp}$ in $\mathcal{I}(\mathcal{R}, \mathcal{S})$ is given by

$$
f_{1}^{\sharp}(q)=\overline{f_{1}\left(t_{q}^{-1}\right)}
$$

or, equivalently, $f_{1}^{\sharp}(g)=\overline{f_{1}\left(g^{-1}\right)}$ for any $g \in G$.

Now take any $f \in \mathcal{I}(\mathcal{R}, \mathcal{S})$ and any $\xi \in \ell^{2}(H \backslash G)$. As in 11.2$)$, define a function $f * \xi$ on $G$ by

$$
(f * \xi)(g)=\sum_{q \in H \backslash G} f\left(g t_{q}^{-1}\right) \xi\left(t_{q}\right) \quad(g \in G) .
$$

It turns out that $f * \xi$ belongs to $\ell^{2}(H \backslash G)$. There exists a number $C_{f}>0$ depending upon $f$ such that $\|f * \xi\|_{2} \leq C_{f}\|\xi\|_{2}$. It follows that, for each $f \in \mathcal{I}(\mathcal{R}, \mathcal{S})$, the equation

$$
\mathcal{L}(f) \xi:=f * \xi \quad\left(\forall \xi \in \ell^{2}(H \backslash G)\right)
$$

defines a bounded operator $\mathcal{L}(f)$ on $\ell^{2}(H \backslash G)$ satisfying $\|\mathcal{L}(f)\| \leq C_{f}$. It is known that $\mathcal{L}: \mathcal{I}(\mathcal{R}, \mathcal{S}) \rightarrow B\left(\ell^{2}(H \backslash G)\right)$ is an involution-preserving representation. Following the terminology in 22, we say that $\mathcal{L}\left(\mathcal{I}_{0}(\mathcal{R}, \mathcal{S})\right)^{\prime \prime}$ is the von Neumann algebra of the Hecke pair $(G, H)$, and denote it by $L(G, H)$.

Let $\rho_{H \backslash G}$ be the unitary representation of $G$ on $\ell^{2}(H \backslash G)$ defined by

$$
\left\{\rho_{H \backslash G}(g) \xi\right\}(q):=\xi(q g) \quad\left(g \in G, q \in H \backslash G, \xi \in \ell^{2}(H \backslash G)\right) .
$$

Then $\rho_{H \backslash G}(G)^{\prime}=L(G, H)$ (cf. [22]). Let $\eta_{0} \in \ell^{2}(H \backslash G)$ be the characteristic function of the singleton $\{H\}$, i.e., $\eta_{0}=\delta_{H}$. It is easy to see that $\eta_{0}$ is a cyclic vector for $\rho_{H \backslash G}(G)^{\prime \prime}$. Hence $\eta_{0}$ is a separating vector for $L(G, H)$. We consider the faithful normal state $\omega_{0}$ on $L(G, H)$ given by

$$
\omega_{0}(T):=\left(T \eta_{0} \mid \eta_{0}\right) \quad(T \in L(G, H)) .
$$

Let $\left\{L(G, H), \pi_{\omega_{0}}, H_{0}\right\}$ be the GNS representation obtained from the state $\omega_{0}$. Note that $\mathcal{L}\left(\mathcal{I}_{0}(\mathcal{R}, \mathcal{S})\right)$ is a $\sigma$-strongly* dense unital $*$-subalgebra of $L(G, H)$ which is globally invariant under the modular automorphism group of $\omega_{0}$ (cf. [22]). It is easy to check that $\Lambda_{\omega_{0}}\left(\mathcal{L}\left(\mathcal{I}_{0}(\mathcal{R}, \mathcal{S})\right)\right.$ ) is dense in $H_{0}$. Now define a linear operator $U_{0}$ from $\Lambda_{\omega_{0}}\left(\mathcal{L}\left(\mathcal{I}_{0}(\mathcal{R}, \mathcal{S})\right)\right)$ into $H_{\Omega}$ by

$$
U_{0} \Lambda_{\omega_{0}}(\mathcal{L}(f)):=\Lambda_{\Omega}(f) \quad\left(f \in \mathcal{I}_{0}(\mathcal{R}, \mathcal{S})\right) .
$$

Since

$$
\begin{aligned}
\left(\Lambda_{\omega_{0}}\left(\mathcal{L}\left(f_{1}\right)\right) \mid \Lambda_{\omega_{0}}\left(\mathcal{L}\left(f_{2}\right)\right)\right) & =\left(\mathcal{L}\left(f_{1}\right) \eta_{0} \mid \mathcal{L}\left(f_{2}\right) \eta_{0}\right)=\sum_{q \in H \backslash G} f_{1}(q) \overline{f_{2}(q)} \\
& =\left(\Lambda_{\Omega}\left(f_{1}\right) \mid \Lambda_{\Omega}\left(f_{2}\right)\right),
\end{aligned}
$$


$U_{0}$ is an isometry with dense range in $H_{\Omega}$. Therefore, $U_{0}$ can be uniquely extended to a unitary $U$ from $H_{0}$ onto $H_{\Omega}$. By definition, it is easy to see that we have $U \pi_{\omega_{0}}(\mathcal{L}(f))=\pi_{\ell}(f) U$ for all $f \in \mathcal{I}_{0}(\mathcal{R}, \mathcal{S})$. It follows that $U \pi_{\omega_{0}}(L(G, H)) U^{*}=$ $\mathcal{H}^{*}(\mathcal{R}, \mathcal{S})$. Therefore, $L(G, H)$ is *-isomorphic to our Hecke von Neumann algebra $\mathcal{H}^{*}(\mathcal{R}, \mathcal{S})$.

Note that, in the situation considered above, there exists a Borel 1-cocycle $c$ from $\mathcal{R}$ into $G$ such that (i) $\operatorname{Ker}(c):=\{(x, y) \in \mathcal{R}: c(x, y)=e\}$ equals $\mathcal{P}$; (ii) $\mathcal{S}$ is equal to the subrelation $c^{-1}(H)$. This can be verified from the discussions in 8 , or [3. Hence we may more generally begin with a Borel 1-cocycle $c$ from the ergodic discrete measured equivalence relation $\mathcal{R}$ into a countable discrete group $G$ such that the asymptotic range of $c$ equals $G$. Moreover, assume that there exists a subgroup $H$ of $G$ such that (i) $(G, H)$ is a Hecke pair; (ii) $\mathcal{S}=c^{-1}(H)$; (iii) $\mathcal{P}_{g}:=c^{-1}\left(H \cap g^{-1} H g\right)$ is ergodic for each $g \in G$. By [8], it is possible to choose choice functions $\left\{\xi_{g}\right\}_{g \in G}$ for $\operatorname{Ker}(c) \subseteq \mathcal{R}$ so that

- $\xi_{g} \in N[\operatorname{Ker}(c)]$ for all $g \in G$ with $\xi_{e}=\mathrm{id}$;

- $\operatorname{Ker}(c)\left(\xi_{s}\left(\xi_{t}(x)\right)\right)=\operatorname{Ker}(c)\left(\xi_{s t}(x)\right)$ for a.e. $x \in X$;

- $c(y, x)=g$ when $y \in \operatorname{Ker}(c)\left(\xi_{g}(x)\right)$;

- $\sigma_{0}(y, x)(s)=s g^{-1}$ for any $s \in G$ when $y \in \operatorname{Ker}(c)\left(\xi_{g}(x)\right)$, where $\sigma_{0}$ is the index cocycle associated with $\left\{\xi_{g}\right\}$.

As before, let $\left\{t_{q}\right\}_{q \in H \backslash G} \subseteq G$ be a set of representatives of the right coset space $H \backslash G$ with $t_{H}=e$. Then it is easy to check that $\left\{\psi_{q}:=\xi_{t_{q}}\right\}_{q \in H \backslash G}$ is a set of choice functions for $\mathcal{S} \subseteq \mathcal{R}$. Denote by $\sigma$ the index cocycle determined by these choice functions. If $q, q^{\prime} \in H \backslash G$ satisfies $\sigma(x, y)(q)=q^{\prime}$ for some $(x, y) \in \mathcal{R}$, then $\left(\psi_{q^{\prime}}(x), \psi_{q}(y)\right) \in \operatorname{Ker}(c)$, so we get

$$
e=c\left(\psi_{q^{\prime}}(x), x\right) c(x, y) c\left(y, \psi_{q}(y)\right)=t_{q^{\prime}} c(x, y)\left(t_{q}\right)^{-1} .
$$

This means that $q^{\prime}=q c(y, x)$. Thus

$$
\sigma(x, y)(q)=q c(y, x) .
$$

Suppose that $F$ is in $L^{\infty}((H \backslash G) \times X)^{\mathcal{S}}$. From the ergodicity of $\mathcal{P}_{t_{q}}$ and 11.4, there exists a unique $f \in \ell^{\infty}(H \backslash G)$ such that $F(q, x)=f(q)$ for a.e. $x \in X$. By ergodicity of $\mathcal{S}, f$ must be $H$-invariant, so $f \in \ell^{\infty}(H \backslash G / H)$. Hence $L^{\infty}((H \backslash G) \times X)^{\mathcal{S}}$ can be identified with $\ell^{\infty}(H \backslash G / H)$. Under this identification, the restriction of the dual operator valued weight $T_{B}$ to $L^{\infty}((H \backslash G) \times X)^{\mathcal{S}}$ is given by

$$
f \in \ell^{\infty}(H \backslash G / H)_{+} \mapsto \sum_{\lambda \in H \backslash G / H} f(\lambda) n_{\lambda} .
$$


This is semifinite, because $(G, H)$ is a Hecke pair. Therefore, it follows from [3] that the commensurability subrelation $\operatorname{Comm}_{\mathcal{R}}(\mathcal{S})$ coincides with $\mathcal{R}$ up to a null set. So $(\mathcal{R}, \mathcal{S})$ too is a Hecke pair. From this point on, by more or less the same arguments as in the previous paragraph, one can show that the Hecke von Neumann algebra $\mathcal{H}^{*}(\mathcal{R}, \mathcal{S})$ associated with $(\mathcal{R}, \mathcal{S})$ is $*$-isomorphic to that of $(G, H)$.

\section{§12. Examples}

In this section, we give some examples of Hecke von Neumann algebras.

(1) We first treat an inclusion of ergodic discrete equivalence relations $\mathcal{S} \subseteq \mathcal{R}$ of finite index. By $[3,(\mathcal{R}, \mathcal{S})$ is a Hecke pair. Moreover, by [12, there exists a canonical system $\{\mathcal{P}, H \subseteq G, \beta\}$ for $\mathcal{S} \subseteq \mathcal{R}$, where $\beta$ is an outer action of a finite group $G$ on $\mathcal{P}$ and $H$ is a subgroup of $G$ such that $H$ contains no nontrivial normal subgroup of $G$. So the Hecke von Neumann algebra $\mathcal{H}^{*}(\mathcal{R}, \mathcal{S})$ is equal to the algebraic Hecke algebra which comes from $(G, H)$.

(2) We shall next show that, for each Hecke pair $(G, H)$, there exists a Hecke pair $(\mathcal{R}, \mathcal{S})$ whose Hecke von Neumann algebra comes from $(G, H)$. Indeed, by using the same arguments as in [3, Section $9(2)]$, we can construct an inclusion of ergodic free group actions $(\mathbb{Z} \subseteq \mathbb{Z} \times H \subseteq \mathbb{Z} \times G)$ on $(X, \mu):=\bigotimes_{n \in \mathbb{Z}}(\Omega, \nu)$, where $(\Omega, \nu):=\bigotimes_{g \in G}\left(\Omega_{g}, \nu_{g}\right), \Omega_{g}=\{0,1\}, \nu_{G}(\{0\})=\nu_{g}(\{1\})=1 / 2$ and $\mathbb{Z}$ and $G$ act as the Bernoulli shifts. Put $(\mathcal{P} \subseteq \mathcal{S} \subseteq \mathcal{R})=\left(\mathcal{R}_{\mathbb{Z}} \subseteq \mathcal{R}_{\mathbb{Z} \times H} \subseteq \mathcal{R}_{\mathbb{Z} \times G}\right)$. By construction, these equivalence relations are ergodic. We define a 1-cocycle $c$ from $\mathcal{R}$ onto $G$ by $c(x,(n, g) x):=g(x \in X,(n, g) \in \mathbb{Z} \times G)$. It is easy to check that $(\mathcal{P} \subseteq \mathcal{S} \subseteq \mathcal{R})$ is equal to $\left(\operatorname{Ker}(c) \subseteq c^{-1}(H) \subseteq c^{-1}(G)\right)$. So, by using the same arguments in the previous section, we conclude that the Hecke von Neumann algebra $\mathcal{H}^{*}(\mathcal{R}, \mathcal{S})$ comes from the Hecke pair $(G, H)$.

\section{Acknowledgements}

The authors are grateful to the referee for his/her careful reading and helpful suggestions to improve their earlier manuscript.

This research was partially supported by Grant-in-Aid for Scientific Research (KAKENHI), 19740090 and 19540206.

\section{References}

[1] H. Aoi, A construction of equivalence subrelations for intermediate subalgebras, J. Math. Soc. Japan 55 (2003), 713-725. Zbl 1033.46046 MR 1978219

[2] A remark on the commensurability for inclusions of ergodic measured equivalence relations, Hokkaido Math. J. 37 (2008), 545-560. Zbl 1151.37006 MR 2441937 
[3] H. Aoi and T. Yamanouchi, On the normalizing groupoids and the commensurability groupoids for inclusions of factors associated to ergodic equivalence relations-subrelations, J. Funct. Anal. 240 (2006), 297-333. Zbl 1122.28012 MR 2261685

[4] J.-B. Bost and A. Connes, Hecke algebras, type III factors and phase transitions with spontaneous symmetry breaking in number theory, Selecta Math. (N.S.) 1 (1995), 411-457. Zbl 0842.46040 MR 1366621

[5] M. Enock and R. Nest, Irreducible inclusions of factors, multiplicative unitaries, and Kac algebras, J. Funct. Anal. 137 (1996), 466-543. Zbl 0847.22003 MR 1387518

[6] J. Feldman and C. C. Moore, Ergodic equivalence relations, cohomology and von Neumann algebras, I, Trans. Amer. Math. Soc. 234 (1977), 289-324. Zbl 0369.22009 MR 0578656

[7] Er E Ergodic equivalence relations, cohomology and von Neumann algebras, II, Trans. Amer. Math. Soc. 234 (1977), 325-359. Zbl 0369.22010 MR 0578730

[8] J. Feldman, C. E. Sutherland and R. J. Zimmer, Subrelations of ergodic equivalence relations, Ergodic Theory Dynam. Systems 9 (1989), 239-269. Zbl 0654.22003 MR 0578730

[9] U. Haagerup, Operator valued weights in von Neumann algebras, I, J. Funct. Anal. 32 (1979), 175-206. Zbl 0426.46046 MR 0534673

[10] 339-361. Zbl 0426.46047 MR 0549119

[11] P. Hahn, The regular representations of measure groupoids, Trans. Amer. Math. Soc. 242 (1978), 35-72. Zbl 0356.46055 MR 0496797

[12] T. Hamachi, Canonical subrelations of ergodic equivalence relations-subrelations, J. Operator Theory 43 (2000), 3-34. Zbl 0990.37001 MR 1740892

[13] M. Izumi, R. Longo and S. Popa, A Galois correspondence for compact groups of automorphisms of von Neumann algebras with a generalization to Kac algebras, J. Funct. Anal. 155 (1998), 25-63. Zbl 0915.46051 MR 1622812

[14] H. Kosaki, Extension of Jones' theory on index to arbitrary factors, J. Funct. Anal. 66 (1986), 123-140. Zbl 0607.46034 MR 0829381

[15] A. Krieg, Hecke algebras, Mem. Amer. Math. Soc. 87 (1990), no. $435 . \quad$ Zbl 0706.11029 MR 1027069

[16] J. Kustermans and S. Vaes, Locally compact quantum groups in the von Neumann algebraic setting, Math. Scand. 92 (2003), 68-92. Zbl $1034.46067 \mid$ MR 1951446

[17] T. Miyake, Modular forms, Springer, Berlin, 1989. Zbl 0701.11014 MR 2194815

[18] Ş. Strătilă, Modular theory in operator algebras, Abacus Press, 1981. Zbl 0504.46043 MR 0696172

[19] Ş. Strătilă and L. Zsidó, Lectures on von Neumann algebras, Abacus Press, 1979. Zbl 0391.46048 MR 0526399

[20] M. Takesaki, Conditional expectations in von Neumann algebras, J. Funct. Anal. 9 (1972), 306-321. Zbl 0245.46089 MR 0303307

[21] _ Theory of operator algebras I, Springer, 1979. Zbl 0436.46043 MR 0548728

[22] K. Tzanev, Hecke $C^{*}$-algebras and amenability, J. Operator Theory 50 (2003), 169-178. Zbl 1036.46054 MR 2015025

[23] T. Yamanouchi, Crossed products by groupoid actions and their smooth flows of weights, Publ. RIMS Kyoto Univ. 28 (1992), 535-578. Zbl 0824.46080 MR 1191875 